\title{
HOMOGENEOUS EINSTEIN METRICS ON GENERALIZED FLAG MANIFOLDS WITH FIVE ISOTROPY SUMMANDS
}

\author{
ANDREAS ARVANITOYEORGOS, IOANNIS CHRYSIKOS, AND YUSUKE SAKANE
}

\begin{abstract}
We construct the homogeneous Einstein equation for generalized flag manifolds $G / K$ of a compact simple Lie group $G$ whose isotropy representation decomposes into five inequivalent irreducible $\operatorname{Ad}(K)$ submodules. To this end we apply a new technique which is based on a fibration of a flag manifold over another flag manifold and the theory of Riemannian submersions. We classify all generalized flag manifolds with five isotropy summands, and we use Gröbner bases to study the corresponding polynomial systems for the Einstein equation. For the generalized flag manifolds $\mathrm{E}_{6} /(\mathrm{SU}(4) \times \mathrm{SU}(2) \times \mathrm{U}(1) \times \mathrm{U}(1))$ and $\mathrm{E}_{7} /(\mathrm{U}(1) \times \mathrm{U}(6))$ we find explicitely all invariant Einstein metrics up to isometry. For the generalized flag manifolds $\mathrm{SO}(2 \ell+1) /(\mathrm{U}(1) \times \mathrm{U}(p) \times \mathrm{SO}(2(\ell-p-1)+1))$ and $\mathrm{SO}(2 \ell) /(\mathrm{U}(1) \times \mathrm{U}(p) \times \mathrm{SO}(2(\ell-p-1)))$ we prove existence of at least two non Kähler-Einstein metrics. For small values of $\ell$ and $p$ we give the precise number of invariant Einstein metrics.
\end{abstract}

2000 Mathematics Subject Classification. Primary 53C25; Secondary 53C30.

Keywords: Homogeneous space, Einstein metric, Riemannian submersion, flag manifold, isotropy representation.

\section{INTRODUCTION}

A Riemannian manifold $(M, g)$ is called Einstein if it has constant Ricci curvature, i.e. $\operatorname{Ric}_{g}=\lambda \cdot g$ for some $\lambda \in \mathbb{R}$. We are concerned with homogeneous Einstein metrics on reductive homogeneous spaces whose isotropy representation decomposes into a direct sum of irreducible non equivalent summands. Then the Einstein equation reduces to a non linear algebraic system of equations. The computation of the Ricci tensor is in general a difficult task, especially when the number of isotropy summands increases. In this paper we introduce a method for computing the Ricci tensor for a homogeneous space via Riemannian submersions, and we apply this for a large class of homogeneous spaces the generalized flag manifolds. These are compact homogeneous spaces of the form $G / K=G / C(S)$, where $G$ is a compact, connected semisimple Lie group and $C(S)$ is the centralizer of a torus $S \subset G$. These spaces exhaust all compact simply connected homogeneous Kähler manifolds of a compact, connected and semisimple Lie group. Their classification is based on the painted Dynkin diagrams (cf. [Ale1], AlAr]) and their Kähler geometry is very interesting on its own right (cf. [AlPe, Bor]). For example, $M=G / K=G / C(S)$ admits a finite number of invariant complex structures, and for each complex structure there is a unique homogeneous Kähler-Einstein metric.

Nowadays, homogeneous Einstein metrics on flag manifolds have been better understood. They have been completely classified for any flag manifold $M=G / K$ (of a compact simple Lie group $G$ ) with two (ACh2,

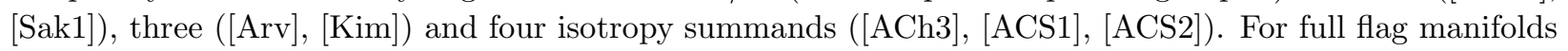
corresponding to classical Lie groups the existence problem has also been studied by several authors (cf. Arv, DSN, Sak2), but a full classification is still unknown, except for some low dimensional cases. On the other hand, in a recent work of the authors (ACS4]) all $\mathrm{G}_{2}$-invariant Einstein metrics were obtained on the exceptional full flag manifold $\mathrm{G}_{2} / T$ (a homogeneous space with six isotropy summands). However, we are still far from general results and a complete classification of invariant Einstein metrics seems to be difficult (by means of the traditional methods).

In the present paper we classify flag manifolds whose isotropy representation decomposes into five irreducible $\operatorname{Ad}(K)$-submodules

$$
\mathfrak{m}=T_{o} M=\mathfrak{m}_{1} \oplus \mathfrak{m}_{2} \oplus \mathfrak{m}_{3} \oplus \mathfrak{m}_{4} \oplus \mathfrak{m}_{5}
$$

The second author was full-supported by Masaryk University under the Grant Agency of Czech Republic, project no. P 201/12/G028. 
and use the new method to compute the Ricci tensor for each of these spaces. Then we study the existence of non Kähler Einstein metrics. The first results about Einstein metrics on flag manifolds with five isotropy summands were obtained in a recent work of the second author [Chr2, where he studied $S O(7)$-invariant Einstein metrics for flag manifolds of the form $S O(7) / K$. Also, in ChSa the last two authors classified all homogeneous Einstein metrics for the (unique) exceptional flag manifold $G / K$ with second Betti number $b_{2}(M)=1$, whose isotropy representation satisfies (11). This flag manifold corresponds to $G=\mathrm{E}_{8}$. As we will see in this paper there are also flag manifolds with five isotropy summands with $b_{2}(M)=2$. In fact the cases $b_{2}(M)=1$ or $b_{2}(M)=2$ exhaust all flag manifolds with five isotropy summands, both classical and exceptional.

Recall that any flag manifold $G / K$ of a compact simple Lie group $G$ is determined by a choice of a pair $\left(\Pi, \Pi_{0}\right)$, where $\Pi$ is a system of simple roots for $G$ and $\Pi_{0} \subset \Pi$. By painting black the nodes in the Dynkin diagram $\Gamma(\Pi)$ of $G$ corresponding to the simple roots of the set $\Pi \backslash \Pi_{0}$, we obtain the painted Dynkin diagram of $G / K$. The semisimple part of the reductive subgroup $K$ is obtained by the subdiagram of white roots, and any black root gives rise to a $\mathrm{U}(1)$-component (the $\mathrm{U}(1)$-components form the center of $K$ whose dimension is equal to the second Betti number of $M$, see Section 3). In terms of painted Dynkin diagrams, flag manifolds $G / K$ of a simple Lie group $G$ whose tangent space $\mathfrak{m}=T_{o}(G / K)$ decomposes as (1) can be obtained as follows:

(a) Paint black one simple root of Dynkin mark 5 , that is

$$
\Pi \backslash \Pi_{0}=\left\{\alpha_{p}: \operatorname{Mrk}\left(\alpha_{p}\right)=5\right\} .
$$

As mentioned earlier, case (a) appears only for $G=\mathrm{E}_{8}$.

(b) Paint black two simple roots, one of Dynkin mark 1 and one of Dynkin mark 2, that is

$$
\Pi \backslash \Pi_{0}=\left\{\alpha_{i}, \alpha_{j}: \operatorname{Mrk}\left(\alpha_{i}\right)=1, \operatorname{Mrk}\left(\alpha_{j}\right)=2\right\} .
$$

(c) Paint black two simple roots both of Dynkin mark 2, that is

$$
\Pi \backslash \Pi_{0}=\left\{\alpha_{i}, \alpha_{j}: \operatorname{Mrk}\left(\alpha_{i}\right)=\operatorname{Mrk}\left(\alpha_{j}\right)=2\right\} .
$$

We call the pairs $\left(\Pi, \Pi_{0}\right)$ arising form cases (b) and (c) as pairs of Type $A$ and Type $B$ respectively. We will use the same name for the corresponding painting Dynkin diagrams and for the flag manifolds determined by them. According to [ACh3, Propositions 5 and 6] if $M=G / K$ is of Type A or B then the corresponding isotropy decomposition is given as follows:

Type $\mathbf{A} \Rightarrow \mathfrak{m}=\mathfrak{m}_{1} \oplus \mathfrak{m}_{2} \oplus \mathfrak{m}_{3} \oplus \mathfrak{m}_{4} \quad$ or $\quad \mathfrak{m}=\mathfrak{m}_{1} \oplus \mathfrak{m}_{2} \oplus \mathfrak{m}_{3} \oplus \mathfrak{m}_{4} \oplus \mathfrak{m}_{5}$.

Type $\mathbf{B} \Rightarrow \mathfrak{m}=\mathfrak{m}_{1} \oplus \mathfrak{m}_{2} \oplus \mathfrak{m}_{3} \oplus \mathfrak{m}_{4} \oplus \mathfrak{m}_{5} \quad$ or $\quad \mathfrak{m}=\mathfrak{m}_{1} \oplus \mathfrak{m}_{2} \oplus \mathfrak{m}_{3} \oplus \mathfrak{m}_{4} \oplus \mathfrak{m}_{5} \oplus \mathfrak{m}_{6}$.

In Table 1 we give the pairs $\left(\Pi, \Pi_{0}\right)$ of Type $\mathrm{A}$ and $\mathrm{B}$, which determine flag manifolds $G / K$ with $\mathfrak{m}=$ $\mathfrak{m}_{1} \oplus \cdots \oplus \mathfrak{m}_{5}$. The explicit form of these flag manifolds is given in Table 2 .

Table 1. Pairs $\left(\Pi, \Pi_{0}\right)$ of Type A and B which determine flag manifolds with five isotropy summnads

\begin{tabular}{|r|l|l|}
\hline Classical Lie group $G$ & $B_{\ell}=\mathrm{SO}(2 \ell+1)$ & $D_{\ell}=\mathrm{SO}(2 \ell)$ \\
\hline Type A & $\Pi \backslash \Pi_{0}=\left\{\alpha_{1}, \alpha_{p+1}: 2 \leq p \leq \ell-1\right\}$ & $\Pi \backslash \Pi_{0}=\left\{\alpha_{1}, \alpha_{p+1}: 2 \leq p \leq \ell-3\right\}$ \\
Type B & $\Pi \backslash \Pi_{0}=\left\{\alpha_{p}, \alpha_{p+1}: 2 \leq p \leq \ell-1\right\}$ & $\Pi \backslash \Pi_{0}=\left\{\alpha_{p}, \alpha_{p+1}: 2 \leq p \leq \ell-3\right\}$ \\
\hline Exceptional Lie group $G$ & $\mathrm{E}_{6}$ & $\mathrm{E}_{7}$ \\
\hline Type A & $\Pi \backslash \Pi_{0}=\left\{\alpha_{1}, \alpha_{4}\right\}$ & $\Pi \backslash \Pi_{0}=\left\{\alpha_{1}, \alpha_{7}\right\}$ \\
Type A & $\Pi \backslash \Pi_{0}=\left\{\alpha_{2}, \alpha_{5}\right\}$ & \\
Type B & $\Pi \backslash \Pi_{0}=\left\{\alpha_{4}, \alpha_{6}\right\}$ & $\Pi \backslash \Pi_{0}=\left\{\alpha_{6}, \alpha_{7}\right\}$ \\
Type B & $\Pi \backslash \Pi_{0}=\left\{\alpha_{2}, \alpha_{6}\right\}$ & \\
\hline
\end{tabular}

\footnotetext{
${ }^{1}$ The Dynkin mark of a simple root $\alpha_{i} \in \Pi(i=1, \ldots, \ell)$ is the positive integer $m_{i}$ in the expression of the highest root $\widetilde{\alpha}=\sum_{k=1}^{\ell} m_{k} \alpha_{k}$ in terms of simple roots. We will denote by Mrk the function Mrk: $\Pi \rightarrow \mathbb{Z}^{+}, \alpha_{i} \mapsto m_{i}$.
} 
For any Lie group $G$ which appears in Table 1, one can see that the flag manifolds $G / K$ of Type A and $\mathrm{B}$ are equivalent to each other (since the isotropy subgroups are conjugate) 2 In fact, in Section 4 we will prove that there is an isometry arising from the action of the Weyl group of $G$ and makes the corresponding flag manifolds $G / K$ of Type A and B isometric to each other, as real manifolds. For this reason there are only four non isometric flag manifolds (as real manifolds) with $b_{2}(M)=2$ with five isotropy summands as shown in Table 2.

Table 2. Generalized flag manifolds with five isotropy summands and $b_{2}(M)=2$

\begin{tabular}{ll}
$M=G / K$ classical & $M=G / K$ exceptional \\
\hline $\mathrm{SO}(2 \ell+1) / \mathrm{U}(1) \times \mathrm{U}(p) \times \mathrm{SO}(2(\ell-p-1)+1)$ & $\mathrm{E}_{6} / \mathrm{SU}(4) \times \mathrm{SU}(2) \times \mathrm{U}(1)^{2}$ \\
$\mathrm{SO}(2 \ell) / \mathrm{U}(1) \times \mathrm{U}(p) \times \mathrm{SO}(2(\ell-p-1))$ & $\mathrm{E}_{7} / \mathrm{SU}(6) \times \mathrm{U}(1)^{2}$
\end{tabular}

The classification of flag manifolds with five isotropy summands is given in Section 4 .

The main difficulty in constructing the Einstein equation for a $G$-invariant metric on a flag manifold in Table 2 is the calculation of the non zero structure constants $\left[\begin{array}{c}k \\ i j\end{array}\right]$ of $G / K$ with respect to the decomposition (11) (see Section 2). A first step towards this procedure is to use the known Kähler-Einstein metric on any flag manifold. This metric can be computed by using the Koszul formula (see Section 5). Secondly, and this is the main contribution of the present paper, we take advantage of a fibration of a flag manifold over another flag manifold and use methods of Riemannian submersions to compare Ricci tensors of total space and base space. In this way we are able to calculate $\left[\begin{array}{l}k \\ i j\end{array}\right]$ in terms of the dimension of the submodules $\mathfrak{m}_{i}$ in the decomposition (1). We point out that this new technique can be useful for the study of homogeneous Einstein metrics for more general homogeneous spaces, whose isotropy representation satisfies certain conditions. In this way the Einstein equation reduces to a polynomial system of four equations in four unknowns. For the exceptional flag manifolds we classify all homogeneous Einstein metrics. For the classical flag manifolds a complete classification of homogeneous Einstein metrics in the general case is a difficult task, because the corresponding systems of equations depend on four positive parameters (which define the invariant Riemannian metric), the Einstein constant $\lambda>0$ and the positive integers $\ell$ and $p$. However, by using Gröbner bases we can show that the equations are reduced to a polynomial equation of one variable and then prove the existence of non Käler Einstein metrics. In fact, this is another contribution of the present paper, because we prove existence of real solutions for polynomial equations whose coefficients depend on parameters $(\ell$ and $p)$.

The paper is organized as follows: In Section 2 we discuss $G$-invariant metrics on homogeneous spaces $G / K$ and compare them with Riemannian submersion metrics for a fibration $L / K \rightarrow G / K \rightarrow G / L$. Then we give expression for the Ricci tensor for a submersion metric. In Section 3 we recall various facts about generalized flag manifolds which will be used in Section 4 for the classification of such spaces with five isotropy summands. Combined with the work ChSa these spaces are $\mathrm{E}_{8} /(\mathrm{U}(1) \times \mathrm{SU}(4) \times \mathrm{SU}(5))$ with second Betti number 1 and the spaces in Table 2 with second Betti number 2. In Section 5 we give the Kähler-Einstein metrics for flag manifolds with five isotropy summands and in Section 6 we compute the Ricci tensor for these spaces by using our method of Riemannian submersions and the known Kähler-Einstein metrics. Section 7 is devoted to the study of the algebraic systems of equations by using Gröbner bases techniques.

TheOrem A. Let $M=G / K$ be one of the flag manifolds $\mathrm{E}_{6} /(\mathrm{SU}(4) \times \mathrm{SU}(2) \times \mathrm{U}(1) \times \mathrm{U}(1))$ or $\mathrm{E}_{7} /(\mathrm{U}(1) \times$ $\mathrm{U}(6))$. Then $M$ admits exactly seven $G$-invariant Einstein metrics up to isometry. There are two KählerEinstein metrics and five non-Kähler Einstein metrics (up to scalar). These metrics are given in Theorems 7.1 and 7.2.

TheOrem B. Let $M=G / K$ be one of the flag manifolds $\mathrm{SO}(2 \ell+1) /(\mathrm{U}(1) \times \mathrm{U}(p) \times \mathrm{SO}(2(\ell-p-1)+1))$ $(\ell \geq 3,3 \leq p \leq \ell-1)$ or $\mathrm{SO}(2 \ell) /(\mathrm{U}(1) \times \mathrm{U}(p) \times \mathrm{SO}(2(\ell-p-1)))(\ell \geq 5,3 \leq p \leq \ell-3)$. Then $M$ admits at least two G-invariant non-Kähler Einstein metrics (cf. Theorem 7.3).

\footnotetext{
${ }^{2}$ Two flag manifolds $G / K$ and $G / K^{\prime}$ are called equivalent if there exists an automorphism $\phi \in \operatorname{Aut}(G)$ such that $\phi(K)=K^{\prime}$. Such an automorphism defines a diffeomorphism $\tilde{\phi}: G / K \rightarrow G / K^{\prime}$ given by $\tilde{\phi}(g K)=\phi(g) K^{\prime}$, which satisfies $\tilde{\phi}(g x)=\phi(g) \tilde{\phi}(x)$ for all $g \in G, x \in G / K$.
} 
Theorem C. Let $M=G / K$ be one of the flag manifolds $\mathrm{SO}(2 \ell+1) /(\mathrm{U}(1) \times \mathrm{U}(2) \times \mathrm{SO}(2 \ell-5))(\ell \geq 6)$ or $\mathrm{SO}(2 \ell) /(\mathrm{U}(1) \times \mathrm{U}(2) \times \mathrm{SO}(2(\ell-3)))(\ell \geq 7)$. Then $M$ admits at least four $G$-invariant non-Kähler Einstein metrics (cf. Theorem 7.4).

Note that the special case $\ell=3, p=2$ (the space $\mathrm{SO}(7) / \mathrm{U}(1) \times \mathrm{U}(2))$ was studied among other results in Chr2. This flag manifold admits (up to isometry) precisely three non-Kähler Einstein metrics and precisely two Kähler-Einstein metrics. For small values of $\ell$ and $p$ it is possible to obtain the precise number of all non isometric invariant Einstein metrics. We discuss this at the end of the paper (cf. Table 4).

\section{Reductive homogeneous spaces and Riemannian submersions}

In this section we describe the Einstein equation for any $G$-invariant metric on a compact reductive homogeneous manifold $G / K$, and give expression of the Ricci tensor of a submersion metric associated to a certain fibration $G / K \rightarrow G / L$. This expression will be used in Chapter 6 to calculate $\left[\begin{array}{c}k \\ i j\end{array}\right]$ for flag manifolds with five isotropy summands.

2.1. The Ricci tensor for a reductive homogeneous spaces. Let $G$ be a compact semisimple Lie group, $K$ a connected closed subgroup of $G$ and let $\mathfrak{g}$ and $\mathfrak{k}$ be the corresponding Lie algebras. The Killing form of $\mathfrak{g}$ is negative definite, so we can define an $\operatorname{Ad}(G)$-invariant inner product $B$ on $\mathfrak{g}$ given by $B=-$ Killing form of $\mathfrak{g}$. Let $\mathfrak{g}=\mathfrak{k} \oplus \mathfrak{m}$ be a reductive decomposition of $\mathfrak{g}$ with respect to $B$ so that $[\mathfrak{k}, \mathfrak{m}] \subset \mathfrak{m}$ and $\mathfrak{m} \cong T_{o}(G / K)$. We assume that $\mathfrak{m}$ admits a decomposition into mutually non equivalent irreducible $\operatorname{Ad}(K)$-modules as follows:

$$
\mathfrak{m}=\mathfrak{m}_{1} \oplus \cdots \oplus \mathfrak{m}_{q}
$$

Then any $G$-invariant metric on $G / K$ can be expressed as

$$
\langle,\rangle=\left.x_{1} B\right|_{\mathfrak{m}_{1}}+\cdots+\left.x_{q} B\right|_{\mathfrak{m}_{q}},
$$

for positive real numbers $\left(x_{1}, \cdots, x_{q}\right) \in \mathbb{R}_{+}^{q}$. Note that $G$-invariant symmetric covariant 2 -tensors on $G / K$ are of the same form as the Riemannian metrics (although they are not necessarilly positive definite). In particular, the Ricci tensor $r$ of a $G$-invariant Riemannian metric on $G / K$ is of the same form as (3), that is

$$
r=\left.y_{1} B\right|_{\mathfrak{m}_{1}}+\cdots+\left.y_{q} B\right|_{\mathfrak{m}_{q}}
$$

for some real numbers $y_{1}, \ldots, y_{q}$.

Let $\left\{e_{\alpha}\right\}$ be a $B$-orthonormal basis adapted to the decomposition of $\mathfrak{m}$, i.e. $e_{\alpha} \in \mathfrak{m}_{i}$ for some $i$, and $\alpha<\beta$ if $i<j$. We put $A_{\alpha \beta}^{\gamma}=B\left(\left[e_{\alpha}, e_{\beta}\right], e_{\gamma}\right)$ so that $\left[e_{\alpha}, e_{\beta}\right]=\sum_{\gamma} A_{\alpha \beta}^{\gamma} e_{\gamma}$ and set $\left[\begin{array}{c}k \\ i j\end{array}\right]=\sum\left(A_{\alpha \beta}^{\gamma}\right)^{2}$, where the sum is taken over all indices $\alpha, \beta, \gamma$ with $e_{\alpha} \in \mathfrak{m}_{i}, e_{\beta} \in \mathfrak{m}_{j}, e_{\gamma} \in \mathfrak{m}_{k}$ (cf. WaZi]). Then the positive numbers $\left[\begin{array}{c}k \\ i j\end{array}\right]$ are independent of the $B$-orthonormal bases chosen for $\mathfrak{m}_{i}, \mathfrak{m}_{j}, \mathfrak{m}_{k}$, and $\left[\begin{array}{c}k \\ i j\end{array}\right]=\left[\begin{array}{c}k \\ j i\end{array}\right]=\left[\begin{array}{c}j \\ k i\end{array}\right]$.

Let $d_{k}=\operatorname{dim} \mathfrak{m}_{k}$. Then we have the following:

Lemma 2.1. ([PaSa] $)$ The components $r_{1}, \ldots, r_{q}$ of the Ricci tensor $r$ of the metric $\langle$,$\rangle of the form (3)$ on $G / K$ are given by

$$
r_{k}=\frac{1}{2 x_{k}}+\frac{1}{4 d_{k}} \sum_{j, i} \frac{x_{k}}{x_{j} x_{i}}\left[\begin{array}{c}
k \\
j i
\end{array}\right]-\frac{1}{2 d_{k}} \sum_{j, i} \frac{x_{j}}{x_{k} x_{i}}\left[\begin{array}{c}
j \\
k i
\end{array}\right] \quad(k=1, \ldots, q),
$$

where the sum is taken over $i, j=1, \ldots, q$.

Since by assumption the submodules $\mathfrak{m}_{i}, \mathfrak{m}_{j}$ in the decomposition (2) are matually non equivalent for any $i \neq j$, it will be $r\left(\mathfrak{m}_{i}, \mathfrak{m}_{j}\right)=0$ whenever $i \neq j$. Thus by Lemma 2.1 it follows that $G$-invariant Einstein metrics on $M=G / K$ are exactly the positive real solutions $g=\left(x_{1}, \ldots, x_{q}\right) \in \mathbb{R}_{+}^{q}$ of the polynomial system $\left\{r_{1}=\lambda, r_{2}=\lambda, \ldots, r_{q}=\lambda\right\}$, where $\lambda \in \mathbb{R}_{+}$is the Einstein constant. 
2.2. Riemannian submersions. Let $G$ be a compact semisimple Lie group and $K, L$ two closed subgroups of $G$ with $K \subset L$. Then there is a natural fibration $\pi: G / K \rightarrow G / L$ with fiber $L / K$.

Let $\mathfrak{p}$ be the orthogonal complement of $\mathfrak{l}$ in $\mathfrak{g}$ with respect to $B$, and $\mathfrak{q}$ be the orthogonal complement of $\mathfrak{k}$ in $\mathfrak{l}$. Then we have $\mathfrak{g}=\mathfrak{l} \oplus \mathfrak{p}=\mathfrak{k} \oplus \mathfrak{q} \oplus \mathfrak{p}$. An $\operatorname{Ad}_{G}(L)$-invariant scalar product on $\mathfrak{p}$ defines a $G$-invariant metric $\check{g}$ on $G / L$, and an $\operatorname{Ad}_{L}(K)$-invariant scalar product on $\mathfrak{q}$ defines an $L$-invariant metric $\hat{g}$ on $L / K$. The orthogonal direct sum for these scalar products on $\mathfrak{q} \oplus \mathfrak{p}$ defines a $G$-invariant metric $g$ on $G / K$, called submersion metric.

Theorem 2.2. [Be, p. 257] The map $\pi$ is a Riemannian submersion from $(G / K, g)$ to $(G / L, \check{g})$ with totally geodesic fibers isometric to $(L / K, \hat{g})$.

Note that $\mathfrak{q}$ is the vertical subspace of the submersion and $\mathfrak{p}$ is the horizontal subspace.

For a Riemannian submersion, O'Neill $[\mathrm{ON}]$ has introduced two tensors $A$ and $T$. Since in our case the fibers are totally geodesic it is $T=0$. We also have that

$$
A_{X} Y=\frac{1}{2}[X, Y]_{\mathfrak{q}} \quad \text { for } X, Y \in \mathfrak{p} .
$$

Let $\left\{X_{i}\right\}$ be an orthonormal basis of $\mathfrak{p}$ and $\left\{U_{j}\right\}$ be an orthonormal basis of $\mathfrak{q}$. For $X, Y \in \mathfrak{p}$ we put $g\left(A_{X}, A_{Y}\right)=\sum_{i} g\left(A_{X} X_{i}, A_{Y} X_{i}\right)$. Then we have that

$$
g\left(A_{X}, A_{Y}\right)=\frac{1}{4} \sum_{i} \hat{g}\left(\left[X, X_{i}\right]_{\mathfrak{q}},\left[Y, X_{i}\right]_{\mathfrak{q}}\right)
$$

Let $r, \check{r}$ be the Ricci tensors of the metrics $g, \check{g}$ respectively. Then we have ([Be, p. 244])

$$
r(X, Y)=\check{r}(X, Y)-2 g\left(A_{X}, A_{Y}\right) \quad \text { for } X, Y \in \mathfrak{p} .
$$

We remark that there is a corresponding expression $r(U, V)$ for vertical vectors, but it does not contribute additional information in our approach.

Let

$$
\mathfrak{p}=\mathfrak{p}_{1} \oplus \cdots \oplus \mathfrak{p}_{\ell}, \quad \mathfrak{q}=\mathfrak{q}_{1} \oplus \cdots \oplus \mathfrak{q}_{s}
$$

be a decomposition of $\mathfrak{p}$ into irreducible $\operatorname{Ad}(L)$-modules and a decomposition of $\mathfrak{q}$ into irreducible $\operatorname{Ad}(K)$ modules respectively, and assume that the $\operatorname{Ad}(L)$-modules $\mathfrak{p}_{j}(j=1, \cdots, \ell)$ are mutually non equivalent. Note that each irreducible component $\mathfrak{p}_{j}$ as $\operatorname{Ad}(L)$-module can be decomposed into irreducible $\operatorname{Ad}(K)$ modules. To compute the values $\left[\begin{array}{l}k \\ i j\end{array}\right]$ for $G / K$, we use information from the Riemannian submersion $\pi:(G / K, g) \rightarrow(G / L, \breve{g})$ with totally geodesic fibers isometric to $(L / K, \hat{g})$. We consider a $G$-invariant metric on $G / K$ defined by a Riemannian submersion $\pi:(G / K, g) \rightarrow(G / L, \check{g})$ given by

$$
g=\left.y_{1} B\right|_{\mathfrak{p}_{1}}+\cdots+\left.y_{\ell} B\right|_{\mathfrak{p}_{\ell}}+\left.z_{1} B\right|_{\mathfrak{q}_{1}}+\cdots+\left.z_{s} B\right|_{\mathfrak{q}_{s}}
$$

for positive real numbers $y_{1}, \cdots, y_{\ell}, z_{1}, \cdots, z_{s}$.

Then we decompose each irreducible component $\mathfrak{p}_{j}$ into irreducible $\operatorname{Ad}(K)$-modules

$$
\mathfrak{p}_{j}=\mathfrak{m}_{j, 1} \oplus \cdots \oplus \mathfrak{m}_{j, k_{j}},
$$

where the $\operatorname{Ad}(K)$-modules $\mathfrak{m}_{j, t}\left(j=1, \cdots, \ell, t=1, \cdots, k_{j}\right)$ are mutually non equivalent and are chosen to be (up to reordering) submodules from the decomposition (2). Then the submersion metric (7) can be written as

$$
g=\left.y_{1} \sum_{t=1}^{k_{1}} B\right|_{\mathfrak{m}_{1, t}}+\cdots+\left.y_{\ell} \sum_{t=1}^{k_{\ell}} B\right|_{\mathfrak{m}_{\ell, t}}+\left.z_{1} B\right|_{\mathfrak{q}_{1}}+\cdots+\left.z_{s} B\right|_{\mathfrak{q}_{s}}
$$

and this is a special case of the $G$-invariant metric (3). 
Lemma 2.3. Let $d_{j, t}=\operatorname{dim} \mathfrak{m}_{j, t}$. The components $r_{(j, t)}\left(j=1, \cdots, \ell, t=1, \cdots, k_{j}\right)$ of the Ricci tensor $r$ for the metric (8) on $G / K$ are given by

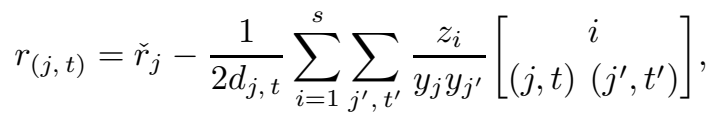

where $\check{r}_{j}$ are the components of Ricci tensor $\check{r}$ for the metric $\check{g}$ on $G / L$.

Proof. Let $\left\{e_{\alpha}^{(j, t)}, e_{\beta}^{(i)}\right\}$ be a $B$-orthonormal basis adapted to the decomposition of $\mathfrak{p} \oplus \mathfrak{q}=\sum_{j} \sum_{t=1}^{k_{j}} \mathfrak{m}_{j, t} \oplus \sum_{i} \mathfrak{q}_{i}$ (with $e_{\alpha}^{(j, t)} \in \mathfrak{m}_{j, t}$ and $e_{\beta}^{(i)} \in \mathfrak{q}_{i}$ ). Put $X_{\alpha}^{(j, t)}=\frac{1}{\sqrt{y_{j}}} e_{\alpha}^{(j, t)}$ and $X_{\beta}^{(i)}=\frac{1}{\sqrt{z_{i}}} e_{\beta}^{(i)}$. Then $\left\{X_{\alpha}^{(j, t)}, X_{\beta}^{(i)}\right\}$ is an orthonormal basis of $\mathfrak{p} \oplus \mathfrak{q}$ for the metric $g$. Then, by using equations (5) and (6), we obtain that

$$
\sum_{\gamma=1}^{d_{j, t}} r\left(X_{\gamma}^{(j, t)}, X_{\gamma}^{(j, t)}\right)=\sum_{\gamma=1}^{d_{j, t}} \check{r}\left(X_{\gamma}^{(j, t)}, X_{\gamma}^{(j, t)}\right)-\frac{1}{2} \sum_{i} \sum_{j^{\prime}, t^{\prime}} \frac{z_{i}}{y_{j} y_{j^{\prime}}}\left[\begin{array}{c}
i \\
(j, t)\left(j^{\prime}, t^{\prime}\right)
\end{array}\right] .
$$

Noting that $\left\{X_{\gamma}^{(j, t)}\right\}_{\gamma=1}^{d_{j, t}}$ is an orthonormal basis of $\mathfrak{m}_{j, t}$, we obtain our claim.

Notice that when metric (7) is viewed as a metric (3) then the horizontal part of $r_{(j, t)}$ equals to $\check{r}_{j}$ $(j=1, \ldots, \ell)$, i.e. it is independent of $t$.

\section{Generalized flag manifolds}

We recall some facts about generalized flag manifolds, concerning painted Dynkin diagrams, isotropy representation and $\mathfrak{t}$-roots. For simplicity we work with simple Lie algebras and groups (the results in the semisimple case are obtained by piecing together the simple factors).

3.1. Description of flag manifolds in terms of painted Dynkin diagrams. Flag manifolds can be described in terms of root systems as follows: Let $G$ be a compact connected simple Lie group with Lie algebra $\mathfrak{g}$, and let $\mathfrak{h}$ a maximal abelian subalgebra of $\mathfrak{g}$. We denote by $\mathfrak{g}^{\mathbb{C}}$ and $\mathfrak{h}^{\mathbb{C}}$ their complexifications and we assume that $\operatorname{dim}_{\mathbb{C}} \mathfrak{h}^{\mathbb{C}}=l=\operatorname{rank} G$. We identify an element of the root system $\Delta$ of $\mathfrak{g}^{\mathbb{C}}$ with respect to the Cartan subalgebra $\mathfrak{h}^{\mathbb{C}}$ with an element of $\mathfrak{h}_{0}=\sqrt{-1} \mathfrak{h}$, by the duality defined by the Killing form of $\mathfrak{g}^{\mathbb{C}}$. This means that for any $\alpha \in \Delta$ we can define $H_{\alpha} \in \mathfrak{h}_{0}$ by $\alpha(H)=B\left(H_{\alpha}, H\right)$ for any $H \in \mathfrak{h}^{\mathbb{C}}$. Consider the root space decomposition of $\mathfrak{g}^{\mathbb{C}}$ relative to $\mathfrak{h}^{\mathbb{C}}$, that is $\mathfrak{g}^{\mathbb{C}}=\mathfrak{h}^{\mathbb{C}} \oplus \sum_{\alpha \in \Delta} \mathfrak{g}_{\alpha}^{\mathbb{C}}$, and let $\Pi=\left\{\alpha_{1}, \ldots, \alpha_{l}\right\}$ be a system of simple roots $\Delta$. We denote by $\left\{\Lambda_{1}, \ldots, \Lambda_{l}\right\}$ the fundamental weights of $\mathfrak{g}^{\mathbb{C}}$ corresponding to $\Pi$, that is $\frac{2\left(\Lambda_{i}, \alpha_{j}\right)}{\left(\alpha_{j}, \alpha_{j}\right)}=\delta_{i j}$ for any $1 \leq i, j \leq l$.

Let $\Pi_{0}$ be a subset of $\Pi$ and set $\Pi_{\mathfrak{m}}=\Pi \backslash \Pi_{0}=\left\{\alpha_{i_{1}}, \ldots, \alpha_{i_{r}}\right\}$, where $1 \leq i_{1}<\cdots<i_{r} \leq l$. We put $\Delta_{0}=\Delta \cap\left\{\Pi_{0}\right\}_{\mathbb{Z}}=\left\{\beta \in \Delta: \beta=\sum_{\alpha_{i} \in \Pi_{0}} k_{i} \alpha_{i}, k_{i} \in \mathbb{Z}\right\}$, where $\left\{\Pi_{0}\right\}_{\mathbb{Z}}$ denotes the set of roots generated by $\Pi_{0}$ with integer coefficients (this is a the subspace of $\mathfrak{h}_{0}$ ). Then $\Delta_{0}$ is a root subsystem of $\Delta$, which means that for any $\alpha, \beta \in \Delta_{0}$ with $\alpha+\beta \in \Delta$ it is also $\alpha+\beta \in \Delta_{0}$. Thus $\Delta_{0}$ generates a maximal complex reductive Lie subalgebra $\mathfrak{k}^{\mathbb{C}}=\mathfrak{h}^{\mathbb{C}} \oplus \sum_{\beta \in \Delta_{0}} \mathfrak{g}_{\beta}^{\mathbb{C}}$ of $\mathfrak{g}^{\mathbb{C}}$, that is $\mathfrak{k}^{\mathbb{C}}=\mathfrak{z} \oplus \mathfrak{k}_{s s}^{\mathbb{C}}$, where $\mathfrak{z}$ is the center of $\mathfrak{k}^{\mathbb{C}}$ and $\mathfrak{k}_{s s}^{\mathbb{C}}=\left[\mathfrak{k}^{\mathbb{C}}, \mathfrak{k}^{\mathbb{C}}\right]$ is its semisimple part. In fact, $\Delta_{0}$ is the root system of $\mathfrak{k}_{s s}^{\mathbb{C}}$, and $\Pi_{0}$ is the correpsonding system of simple roots. Thus we can obtain the decomposition $\mathfrak{k}_{s s}^{\mathbb{C}}=\mathfrak{h}_{K}^{\mathbb{C}} \oplus \sum_{\alpha \in \Delta_{0}} \mathfrak{g}_{\alpha}^{\mathbb{C}}$. Here $\mathfrak{h}_{K}^{\mathbb{C}}=\operatorname{span}_{\mathbb{C}}\left\{H_{\alpha}: \alpha \in \Pi_{0}\right\} \subset \mathfrak{h}^{\mathbb{C}}$ is the Cartan subalgebra of $\mathfrak{k}_{s s}^{\mathbb{C}}$ in $\mathfrak{h}^{\mathbb{C}}$. Note that the center $\mathfrak{z}$ (always non trivial) can be considered as the orthogonal complement of $\mathfrak{h}_{K}^{\mathbb{C}}$ in $\mathfrak{h}^{\mathbb{C}}$ (with respect to the Killing form), that is $\mathfrak{h}^{\mathbb{C}}=\mathfrak{h}_{K}^{\mathbb{C}} \oplus \mathfrak{z}$.

Definition 3.1. The roots of the set $\Delta_{\mathfrak{m}}=\Delta \backslash \Delta_{0}$ are called complemetary roots.

Note that $\Delta_{\mathfrak{m}}$ is not a root system in general. Choose a system of positive roots $\Delta^{+}$for $\mathfrak{g}^{\mathbb{C}}$ with respect to $\Pi$ and set $\Delta_{\mathfrak{m}}^{ \pm}=\Delta^{ \pm} \backslash \Delta_{0}^{ \pm}$, where $\Delta_{0}^{ \pm}=\Delta^{ \pm} \cap\left\{\Pi_{0}\right\}_{\mathbb{Z}}$ and $\Delta^{-}=\left\{-\alpha: \alpha \in \Delta^{+}\right\}$. Then, the set $\Delta_{\Pi_{0}}=\Delta_{0}^{-} \cup \Delta^{+}=\Delta_{0} \cup\left(\Delta^{+} \backslash \Delta_{0}^{+}\right)=\Delta_{0} \cup \Delta_{\mathfrak{m}}^{+}$is a root subsystem of $\Delta$ ([Ale1, p. 16]) and the subalgebra

$$
\mathfrak{p}_{\Pi_{0}}=\mathfrak{h}^{\mathbb{C}} \oplus \sum_{\alpha \in \Delta_{0}^{-} \cup \Delta^{+}} \mathfrak{g}_{\alpha}^{\mathbb{C}}=\mathfrak{h}^{\mathbb{C}} \oplus \sum_{\alpha \in \Delta_{0} \cup \Delta_{\mathfrak{m}}^{+}} \mathfrak{g}_{\alpha}^{\mathbb{C}}=\mathfrak{h}^{\mathbb{C}} \oplus \sum_{\alpha \in \Delta_{0}} \mathfrak{g}_{\alpha}^{\mathbb{C}} \oplus \sum_{\alpha \in \Delta_{\mathfrak{m}}^{+}} \mathfrak{g}_{\alpha}^{\mathbb{C}}
$$


is a parabolic subalgebra of $\mathfrak{g}^{\mathbb{C}}$, since it contains the Borel subalgebra $\mathfrak{b}=\mathfrak{h}^{\mathbb{C}} \oplus \sum_{\alpha \in \Delta^{+}} \mathfrak{g}_{\alpha}^{\mathbb{C}} \subset \mathfrak{g}^{\mathbb{C}}$. In particular, we have a direct decomposition $\mathfrak{p}_{\Pi_{0}}=\mathfrak{k}^{\mathbb{C}} \oplus \mathfrak{r}$, where $\mathfrak{r}=\sum_{\alpha \in \Delta_{m}^{+}} \mathfrak{g}_{\alpha}^{\mathbb{C}}$ is the nilradical of $\mathfrak{p}$ (a regular nilpotent subalgebra of $\mathfrak{g}^{\mathbb{C}}$ ). It is known that any parabolic subalgebra is conjugate to a subalgebra of the form $\mathfrak{p}_{\Pi_{0}}$ for some subset $\Pi_{0} \subset \Pi$, (cf. [Ale1], GOV]). Note that the cases $\Pi_{0}=\emptyset$ and $\Pi_{0}=\Pi$ define the spaces $\mathfrak{b}$ and $\mathfrak{g}^{\mathbb{C}}$ respectively. In this way we can construct a flag manifold $M=G^{\mathbb{C}} / P$, where $G^{\mathbb{C}}$ is the simply connected complex simple Lie group whose Lie algebra is $\mathfrak{g}^{\mathbb{C}}$ and $P \subset G^{\mathbb{C}}$ is the parabolic subgroup generated by $\mathfrak{p}_{\Pi_{0}}$. Since $P$ is always connected, the flag manifold is a (compact) simply connected complex homogeneous manifold. The real representation $M=G / K=G / C(S)$ is obtained by the transitive action of $G$ on $M=G^{\mathbb{C}} / P$, where the close connected subgroup $K=P \cap G$ is identified with the centralizer $C(S)$ of a torus $S \subset G$ (cf. Ale1, GOV]). Thus we always have $\operatorname{rk} G=\operatorname{rk} K$.

Fix now a Weyl basis $E_{\alpha} \in \mathfrak{g}_{\alpha}^{\mathbb{C}}(\alpha \in \Delta)$ with

$$
\begin{aligned}
& {\left[E_{\alpha}, E_{-\alpha}\right]=-H_{\alpha}(\alpha \in \Delta)} \\
& {\left[E_{\alpha}, E_{\beta}\right]=\left\{\begin{array}{lll}
N_{\alpha, \beta} E_{\alpha+\beta} & \text { if } & \alpha+\beta \in \Delta \\
0 & \text { if } & \alpha+\beta \notin \Delta,
\end{array}\right.}
\end{aligned}
$$

where $N_{\alpha, \beta}=N_{-\alpha,-\beta} \in \mathbb{R}$. Then we have

$$
\mathfrak{g}=\mathfrak{h}+\sum_{\alpha \in \Delta}\left\{\mathbb{R}\left(E_{\alpha}+E_{-\alpha}\right)+\mathbb{R} \sqrt{-1}\left(E_{\alpha}-E_{-\alpha}\right)\right\}
$$

The Lie algebra $\mathfrak{k}=\mathfrak{p}_{\Pi_{0}} \cap \mathfrak{g}$ of the isotropy subgroup $K$ is a Lie subalgebra of $\mathfrak{g}$, given by

$$
\mathfrak{k}=\mathfrak{h}+\sum_{\alpha \in \Delta_{0}^{+}}\left\{\mathbb{R}\left(E_{\alpha}+E_{-\alpha}\right)+\mathbb{R} \sqrt{-1}\left(E_{\alpha}-E_{-\alpha}\right)\right\} .
$$

As a real reductive subalgebra, $\mathfrak{k}$ decomposes into a direct sum of its center $\mathfrak{t}$ and its semisimple part $[\mathfrak{k}, \mathfrak{k}]$. Note that

$$
\mathfrak{t}=\mathfrak{z} \cap \mathfrak{h}_{0}=\left\{H \in \mathfrak{h}_{0}:\left(H, \Pi_{0}\right)=0\right\},
$$

where ( , ) denotes the inner product on $\mathfrak{h}_{0}$ (or on the dual space $\mathfrak{h}_{0}^{*}$ ) induced by the Killing form and $\mathfrak{z}$ is the center of $\mathfrak{k}^{\mathbb{C}}$. One can also show that the fundamental weights $\left\{\Lambda_{i_{1}}, \cdots, \Lambda_{i_{r}}\right\}$ form a basis of $\mathfrak{t}$ and that $\mathfrak{t}$ is a real form of $\mathfrak{z}$. If we set $\mathfrak{s}=\sqrt{-1} \mathfrak{t}$ then $\mathfrak{k}$ is given by $\mathfrak{k}=\mathfrak{z}(\mathfrak{s})$ (the Lie algebra of the centralizer of a torus $S$ in $G$ ).

All information which is contained in the pair $\left(\Pi, \Pi_{0}\right)$ can be presented graphicaly by the painted Dynkin diagram of $M=G^{\mathbb{C}} / P=G / K$, which is defined as follows:

Definition 3.2. Let $\Gamma(\Pi)$ be the Dynkin diagram of $\Pi$. By painting black in $\Gamma(\Pi)$ the simple roots $\alpha_{i} \in$ $\Pi_{\mathfrak{m}}=\Pi \backslash \Pi_{0}$ we obtain the painted Dynkin diagram $\Gamma\left(\Pi_{\mathfrak{m}}\right)$ of $M$.

The isotropy subgroup $K$ can be determined from the painted Dynkin diagram $\Gamma\left(\Pi_{\mathfrak{m}}\right)$ as follows: its semisimple part is defined by the subdiagram of white roots (which is not necessarily connected), and each black root gives rise to a $\mathrm{U}(1)$-component which determines the center $Z(K)$ of $K$. We will often make use of the diffeomorphism $\mathrm{SU}(n) \times \mathrm{U}(1) \cong \mathrm{U}(n)$.

3.2. Isotropy summands, t-roots and $G$-invariant Riemannian metrics. Following the notation of the previous paragraph, we assume that a flag manifold $M=G^{\mathbb{C}} / P=G / K$ is defined by a subset $\Pi_{0} \subset \Pi$, such that $\Pi_{\mathfrak{m}}=\Pi \backslash \Pi_{0}=\left\{\alpha_{i_{1}}, \ldots, \alpha_{i_{r}}\right\}$, where $1 \leq i_{1}<\cdots<i_{r} \leq l$, and let $\mathfrak{g}=\mathfrak{k} \oplus \mathfrak{m}$ be a reductive decomposition of the Lie algebra $\mathfrak{g}$ with respect $B$. We identify the isotropy representation $\chi: K \rightarrow \mathrm{GL}(\mathfrak{m})$ of $G / K$ with the adjoint representation $\left.\mathrm{Ad}\right|_{K}$ restricted to $\mathfrak{m}$. In view of relations (11), (12) and the splitting $\Delta_{\mathfrak{m}}^{+}=\Delta^{+} \backslash \Delta_{0}^{+}$it follows that

$$
\mathfrak{m}=\sum_{\alpha \in \Delta_{\mathfrak{m}}^{+}}\left\{\mathbb{R}\left(E_{\alpha}+E_{-\alpha}\right) \oplus \mathbb{R} \sqrt{-1}\left(E_{\alpha}-E_{-\alpha}\right)\right\} .
$$

Thus a basis of $\mathfrak{m}$ consists of the vectors $\left\{A_{\alpha}=\left(E_{\alpha}+E_{-\alpha}\right), B_{\alpha}=\sqrt{-1}\left(E_{\alpha}-E_{-\alpha}\right): \alpha \in \Delta_{\mathfrak{m}}^{+}\right\}$. 
For integers $j_{1}, \ldots, j_{r}$ with $\left(j_{1}, \ldots, j_{r}\right) \neq(0, \ldots, 0)$ we set

$$
\Delta^{\mathfrak{m}}\left(j_{1}, \ldots, j_{r}\right)=\left\{\sum_{j=1}^{l} m_{j} \alpha_{j} \in \Delta^{+}: m_{i_{1}}=j_{1}, \ldots, m_{i_{r}}=j_{r}\right\} \subset \Delta^{+} .
$$

Then it is $\Delta_{\mathfrak{m}}^{+}=\Delta^{+} \backslash \Delta_{0}^{+}=\bigcup_{j_{1}, \ldots, j_{r}} \Delta^{\mathfrak{m}}\left(j_{1}, \ldots, j_{r}\right)$. For $\Delta^{\mathfrak{m}}\left(j_{1}, \ldots, j_{r}\right) \neq \emptyset$ we define an $\operatorname{Ad}(K)$-invariant subspace $\mathfrak{m}\left(j_{1}, \ldots, j_{r}\right)$ of $\mathfrak{g}$ by

$$
\mathfrak{m}\left(j_{1}, \ldots, j_{r}\right)=\sum_{\alpha \in \Delta^{\mathfrak{m}}\left(j_{1}, \ldots, j_{r}\right)}\left\{\mathbb{R} A_{\alpha}+\mathbb{R} B_{\alpha}\right\} .
$$

Thus we have a decomposition of $\mathfrak{m}$ into mutually non equivalent irreducible $\operatorname{Ad}_{G}(K)$-modules $\mathfrak{m}\left(j_{1}, \ldots, j_{r}\right)$ as $\mathfrak{m}=\sum_{j_{1}, \ldots, j_{r}} \mathfrak{m}\left(j_{1}, \ldots, j_{r}\right)$.

We consider the restriction map $\kappa: \mathfrak{h}_{0}^{*} \rightarrow \mathfrak{t}^{*},\left.\alpha \mapsto \alpha\right|_{\mathfrak{t}}$ and note that this is a linear map. We set $\Delta_{\mathfrak{t}}=\kappa(\Delta), \kappa\left(\Delta_{0}\right)=0$.

Definition 3.3. The elements of $\Delta_{\mathfrak{t}}$ are called $\mathfrak{t}$-roots.

Let $\mathfrak{m}^{\mathbb{C}}=T_{o}(G / K)^{\mathbb{C}}$ be the complexification of $\mathfrak{m}$. Then it is $\mathfrak{m}^{\mathbb{C}}=\sum_{\alpha \in \Delta_{\mathfrak{m}}} \mathfrak{g}_{\alpha}^{\mathbb{C}}$ and thus a basis of $\mathfrak{m}^{\mathbb{C}}$ is given by the root vectors $\left\{E_{\alpha}: \alpha \in \Delta_{\mathfrak{m}}\right\}$.

Proposition 3.4. ([Ale1], AlPe] There exists a 1-1 correspondence between $\mathfrak{t}$-roots $\xi$ and irreducible submodules $\mathfrak{m}_{\xi}$ of the $A d_{G}(K)$-module $\mathfrak{m}^{\mathbb{C}}$ given by

$$
\Delta_{\mathfrak{t}} \ni \xi \mapsto \mathfrak{m}_{\xi}=\sum_{\left\{\alpha \in \Delta_{\mathfrak{m}: \kappa(\alpha)=\xi\}}\right.} \mathfrak{g}_{\alpha}^{\mathbb{C}}
$$

By using Proposition 3.4 and the definition of $\mathfrak{t}$-roots, it follows that the $\operatorname{Ad}_{G}(K)$-module $\mathfrak{m}^{\mathbb{C}}$ admits the decomposition $\mathfrak{m}^{\mathbb{C}}=\sum_{\xi \in \Delta_{\mathfrak{t}}} \mathfrak{m}_{\xi}$. If we denote by $\Delta_{\mathfrak{t}}^{+}$the set of all positive $\mathfrak{t}$-roots (this is the restricton of the root system $\Delta^{+}$under the map $\kappa$ ), then the nilradical is given by $\mathfrak{r}=\sum_{\xi \in \Delta_{\mathfrak{t}}^{+}} \mathfrak{m}_{\xi}$.

In order to obtain a decomposition of the real $\operatorname{Ad}(K)$-module $\mathfrak{m}$ in terms of $\mathfrak{t}$-roots, we use the complex conjugation $\tau$ of $\mathfrak{g}^{\mathbb{C}}$ with respect to $\mathfrak{g}$ (note that $\tau$ interchanges $\mathfrak{g}_{\alpha}^{\mathbb{C}}$ and $\mathfrak{g}_{-\alpha}^{\mathbb{C}}$ ). For a complex subspace $W$ of $\mathfrak{g}^{\mathbb{C}}$ we denote by $W^{\tau}$ the set of all fixed points of $\tau$. Then

$$
\mathfrak{m}=\sum_{\xi \in \Delta_{\mathrm{t}}^{+}}\left(\mathfrak{m}_{\xi} \oplus \mathfrak{m}_{-\xi}\right)^{\tau}
$$

Let $\Delta_{\mathfrak{t}}^{+}=\left\{\xi_{1}, \ldots, \xi_{q}\right\}$. Then Proposition 3.4 and relations (13), (14) imply that each real irreducible $\operatorname{ad}(\mathfrak{k})$-submodule $\mathfrak{m}_{i}=\left(\mathfrak{m}_{\xi_{i}} \oplus \mathfrak{m}_{-\xi_{i}}\right)^{\tau}(1 \leq i \leq q)$ corresponding to the positive $\mathbf{t}$-root $\xi_{i}$ is given by

$$
\mathfrak{m}_{i}=\sum_{\left\{\alpha \in \Delta_{\mathfrak{m}}^{+}: \kappa(\alpha)=\xi_{i}\right\}}\left\{\mathbb{R}\left(E_{\alpha}+E_{-\alpha}\right)+\mathbb{R} \sqrt{-1}\left(E_{\alpha}-E_{-\alpha}\right)\right\} .
$$

The results obtained in the previous discussion are summarized in the following:

Proposition 3.5. Let $M=G / K$ be a generalized flag manifold defined by a subset $\Pi_{0} \subset \Pi$ such that $\Pi_{\mathfrak{m}}=\Pi \backslash \Pi_{0}=\left\{\alpha_{i_{1}}, \ldots, \alpha_{i_{r}}\right\}$ with $1 \leq i_{1} \leq \cdots \leq i_{r} \leq \ell$. Assume that $\mathfrak{g}=\mathfrak{k} \oplus \mathfrak{m}$ is a B-orthogonal reductive decomposition. Then

1) There exists a natural one-to-one correspondence between elements of the set $\Delta^{\mathfrak{m}}\left(j_{1}, \ldots, j_{r}\right) \neq \emptyset$ and the set of positive $\mathfrak{t}$-roots $\Delta_{\mathfrak{t}}^{+}=\left\{\xi_{1}, \ldots, \xi_{q}\right\}$. Thus there is a decomposition of $\mathfrak{m}$ into $q$ mutually non-equivalent irreducible $\operatorname{Ad}(K)$-modules

$$
\mathfrak{m}=\sum_{\xi \in \Delta_{\mathfrak{t}+}}\left(\mathfrak{m}_{\xi} \oplus \mathfrak{m}_{-\xi}\right)^{\tau}=\sum_{i=1}^{q}\left(\mathfrak{m}_{\xi_{i}} \oplus \mathfrak{m}_{-\xi_{i}}\right)^{\tau}=\sum_{j_{1}, \ldots, j_{r}} \mathfrak{m}\left(j_{1}, \ldots, j_{r}\right),
$$

for appropriate positive integers $j_{1}, \ldots, j_{r}$. 
2) The dimensions of the real $\operatorname{Ad}(K)$-modules $\mathfrak{m}_{i}(i=1, \ldots, q)$ corresponding to the $\mathfrak{t}$-root $\xi_{i} \in \Delta_{\mathfrak{t}}^{+}$are given by $\operatorname{dim}_{\mathbb{R}} \mathfrak{m}_{i}=2 \cdot\left|\left\{\alpha \in \Delta_{\mathfrak{m}}^{+}: \kappa(\alpha)=\xi_{i}\right\}\right|=2 \cdot\left|\Delta^{\mathfrak{m}}\left(j_{1}, \ldots, j_{r}\right)\right|$, for appropriate positive integers $j_{1}, \ldots, j_{r} 3$

3) Any $G$-invariant Riemannian metric $g$ on $G / K$ can be expressed as

$$
g=\left.\sum_{\xi \in \Delta_{\mathfrak{t}}^{+}} x_{\xi} B\right|_{\left(\mathfrak{m}_{\xi}+\mathfrak{m}_{-\xi}\right)^{\tau}}=\left.\sum_{i=1}^{q} x_{\xi_{i}} B\right|_{\left(\mathfrak{m}_{\xi_{i}}+\mathfrak{m}_{-\xi_{i}}\right)^{\tau}}=\left.\sum_{j_{1}, \ldots, j_{r}} x_{j_{1} \cdots j_{r}} B\right|_{\mathfrak{m}\left(j_{1}, \ldots, j_{r}\right)}
$$

for positive real numbers $x_{\xi}, x_{\xi_{i}}, x_{j_{1} \cdots j_{r}}$. Thus $G$-invariant Riemannian metrics on $M=G / K$ are parametrized by q real positive parameters.

We now show how we can find explicitly the set of $\mathfrak{t}$-roots $\Delta_{\mathfrak{t}}$. Let $\Pi_{\mathfrak{t}}=\left\{\bar{\alpha}_{i_{j}}=\left.\alpha_{i_{j}}\right|_{\mathfrak{t}}: \alpha_{i_{j}} \in \Pi_{\mathfrak{m}}\right\}$. This set is a basis of $\mathfrak{t}^{*}$ in the sense that any $\mathfrak{t}$-root can be written as a linear combination of its elements with integer coefficients of the same sign. In particular, by using the fact that $\kappa\left(\Delta_{0}\right)=0$ we have that

$$
\kappa(\alpha)=k_{i_{1}} \bar{\alpha}_{i_{1}}+\cdots+k_{i_{r}} \bar{\alpha}_{i_{r}}, \quad\left(\alpha \in \Delta_{\mathfrak{m}}^{+}\right) .
$$

Here the positive integers $k_{i_{j}}$ satisfy $0 \leq k_{i_{j}} \leq m_{i_{j}}$, where $m_{i_{j}}$ is the Dynkin mark of the simple root $\alpha_{i_{j}} \in \Pi_{\mathfrak{m}}$, and are not simultaneously zero. Therefore, by using the expressions of the complementary roots in terms of simple roots, and applying formula (17), we can easily determine all positive $\mathbf{t}$-roots. Elements of $\Pi_{\mathfrak{t}}$ are called simple $\mathfrak{t}$-roots and they generalize the notion of simple roots (this means that a simple $\mathfrak{t}$-root $\kappa\left(\alpha_{i_{j}}\right)=\left.\alpha_{i_{j}}\right|_{\mathfrak{t}}=\bar{\alpha}_{i_{j}} \in \Pi_{\mathfrak{t}}$ is a positive $\mathfrak{t}$-root, which can not be written as the sum of two positive $\mathfrak{t}$-roots).

Example 3.6. Flag manifolds of $C_{\ell}=\operatorname{Sp}(\ell)$ (ACS3]). Consider the flag manifolds $M=G / K=$ $\mathrm{Sp}(\ell) /(\mathrm{U}(p) \times \mathrm{U}(q) \times \mathrm{Sp}(\ell-p-q))$ with $\ell \geq 3$ and $1 \leq p, q, p+q \leq \ell-1$. This space is defined by the painted Dynkin diagram $\Gamma\left(\Pi_{\mathfrak{m}}\right)$ with $\Pi_{\mathfrak{m}}=\left\{\alpha_{p}, \alpha_{p+q}: \operatorname{Mrk}\left(\alpha_{p}\right)=\operatorname{Mrk}\left(\alpha_{p+q}\right)=2\right\}$ that is

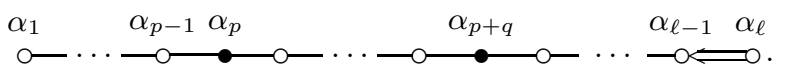

and thus $M$ is of Type B (cf. Introduction). We will show that $\mathfrak{m}=T_{o} M$ decomposes into a direct sum of six pairwise inequivalent $\operatorname{Ad}(K)$-submodules, thus $M$ does not appear in Table 2. Following the notation of [AlAr, p. 3781], we consider an orthonormal basis of $\mathbb{R}^{\ell}$ given by $\left\{e_{i}^{1}, e_{j}^{2}, \pi_{k}\right\}$ with $1 \leq i \leq p$ and $1 \leq j \leq q$ and $1 \leq k \leq \ell-p-q$. Then a system of positive roots for $C_{\ell}$ is given by

$$
\begin{aligned}
\Delta^{+}= & \left\{e_{i}^{1} \pm e_{j}^{1}: 1 \leq i<j \leq p\right\} \cup\left\{e_{i}^{1} \pm e_{j}^{2}: 1 \leq i \leq p, 1 \leq j \leq q\right\} \cup\left\{e_{i}^{2} \pm e_{j}^{2}: 1 \leq i<j \leq q\right\} \\
& \cup\left\{e_{i}^{1} \pm \pi_{k}: 1 \leq i \leq p, 1 \leq k \leq \ell-p-q\right\} \cup\left\{e_{j}^{2} \pm \pi_{k}: 1 \leq j \leq q, 1 \leq k \leq \ell-p-q\right\} \\
& \cup\left\{\pi_{i} \pm \pi_{j}: 1 \leq i<j \leq \ell-p-q\right\} \cup\left\{2 e_{i}^{1}, 2 e_{j}^{2}, 2 \pi_{k}\right\} .
\end{aligned}
$$

A basis of simple roots is given by

$$
\begin{aligned}
\Pi= & \left\{\alpha_{1}=e_{1}^{1}-e_{2}^{1}, \ldots, \alpha_{p-1}=e_{p-1}^{1}-e_{p}^{1}, \alpha_{p}=e_{p}^{1}-e_{1}^{2}\right\} \\
& \cup\left\{\alpha_{p+1}=e_{1}^{2}-e_{2}^{2}, \ldots, \alpha_{p+q-1}=e_{q-1}^{2}-e_{q}^{2}, \ldots, \alpha_{p+q}=e_{q}^{2}-\pi_{1}\right\} \\
& \cup\left\{\phi_{1}=\pi_{1}-\pi_{2}, \ldots, \phi_{\ell-p-q-1}=\pi_{\ell-p-q-1}-\pi_{\ell-p-q}, \phi_{\ell-p-q}=2 \pi_{\ell-p-q}\right\} .
\end{aligned}
$$

The root system of the semisimple part of the isotropy subgroup $K$ is given by

$$
\Delta_{0}^{+}=\left\{e_{i}^{1}-e_{j}^{1}: 1 \leq i<j \leq p\right\} \cup\left\{e_{i}^{2}-e_{j}^{2}: 1 \leq i<j \leq q\right\} \cup\left\{\pi_{i} \pm \pi_{j}, 2 \pi_{k}: 1 \leq i<j \leq \ell-p-q\right\},
$$

thus the positive complementary roots are $\Delta_{\mathfrak{m}}^{+}=\left\{e_{i}^{1}+e_{j}^{1}, e_{i}^{1} \pm e_{j}^{2}, e_{i}^{2}+e_{j}^{2}, e_{i}^{1} \pm \pi_{k}, e_{j}^{2} \pm \pi_{k}, 2 e_{i}^{1}, 2 e_{j}^{2}\right\}$. Let $\alpha=\sum_{k=1}^{\ell} c_{k} \alpha_{k} \in \Delta_{\mathfrak{m}}^{+}$. Since $\Pi_{\mathfrak{m}}=\left\{\alpha_{p}, \alpha_{p+q}\right\}$, by applying relation (17) we obtain that $\kappa(\alpha)=$ $c_{p} \bar{\alpha}_{p}+c_{p+q} \bar{\alpha}_{p+q} \in \Delta_{\mathfrak{t}}^{+}$. Here the coefficients $c_{p}, c_{p+q}$ are such that $0 \leq c_{p}, c_{p+q} \leq 2$, and they are not

\footnotetext{
${ }^{3}$ We denote by $|S|$ the cardinality of a finite set $S$.
} 
simultaneously equal to zero. In particular, by expressing the positive complementary roots in terms of the simple roots we obtain that

$$
\begin{aligned}
& \kappa\left(e_{i}^{1}+e_{j}^{1}\right)=\kappa\left(\alpha_{i}+\cdots+\alpha_{j}+2 \alpha_{j+1}+\cdots+2 \alpha_{p}+\cdots+2 \alpha_{p+q}+2 \phi_{1}+\cdots+\phi_{\ell-p-q}\right) \\
& =2 \bar{\alpha}_{p}+2 \bar{\alpha}_{q}, \\
& \kappa\left(e_{i}^{1}+e_{j}^{2}\right)=\kappa\left(\alpha_{i}+\cdots+\alpha_{p}+\cdots+\alpha_{p+j}+2 \alpha_{p+j+1}+\cdots+2 \alpha_{p+q}+2 \phi_{1}+\cdots+\phi_{\ell-p-q}\right) \\
& =\bar{\alpha}_{p}+2 \bar{\alpha}_{p+q}, \\
& \kappa\left(e_{i}^{1}-e_{j}^{2}\right)=\kappa\left(\alpha_{i}+\cdots+\alpha_{p}+\cdots+\alpha_{p+j}\right)=\bar{\alpha}_{p}, \\
& \kappa\left(e_{i}^{2}+e_{j}^{2}\right) \stackrel{i \gg p}{=} \kappa\left(\alpha_{i}+\cdots+\alpha_{j}+2 \alpha_{j+1}+\cdots+2 \alpha_{p+q}+2 \phi_{1}+\cdots+\phi_{\ell-p-q}\right)=2 \bar{\alpha}_{p+q}, \\
& \kappa\left(e_{i}^{1}-\pi_{k}\right)=\kappa\left(\alpha_{i}+\cdots+\alpha_{p}+\cdots+\alpha_{p+q}+\phi_{1}+\cdots+\phi_{k-1}\right)=\bar{\alpha}_{p}+\bar{\alpha}_{p+q} \\
& \kappa\left(e_{i}^{1}+\pi_{k}\right)=\kappa\left(\alpha_{i}+\cdots+\alpha_{p}+\cdots+\alpha_{p+q}+\phi_{1}+\cdots+\phi_{k-1}+2 \phi_{k}+\cdots+\phi_{\ell-p-q}\right)=\bar{\alpha}_{p}+\bar{\alpha}_{p+q} \text {, } \\
& \kappa\left(e_{j}^{2}-\pi_{k}\right) \stackrel{j \geq p}{=} \kappa\left(\alpha_{j}+\cdots \alpha_{p+q}+\phi_{1}+\cdots+\phi_{k-1}\right)=\bar{\alpha}_{p+q}, \\
& \kappa\left(e_{j}^{2}+\pi_{k}\right) \stackrel{j \gg p}{=} \kappa\left(\alpha_{j}+\cdots \alpha_{p+q}+\phi_{1}+\cdots+\phi_{k-1}+2 \phi_{k}+\cdots+\phi_{\ell-p-q}\right)=\bar{\alpha}_{p+q}, \\
& \kappa\left(2 e_{i}^{1}\right)=\kappa\left(2 \alpha_{i}+\cdots+2 \alpha_{p}+\cdots+2 \alpha_{p+q}+2 \phi_{1}+\cdots+2 \phi_{\ell-p-q-1}+\phi_{\ell-p-q}\right)=2 \bar{\alpha}_{p}+2 \bar{\alpha}_{p+q}, \\
& \kappa\left(2 e_{j}^{2}\right) \stackrel{j \gg p}{=} \kappa\left(2 \alpha_{j}+\cdots+2 \alpha_{p+q}+2 \phi_{1}+\cdots+2 \phi_{\ell-p-q-1}+\phi_{\ell-p-q}\right)=2 \bar{\alpha}_{p+q} .
\end{aligned}
$$

Thus the set of $\mathfrak{t}$-roots is given by $\Delta_{\mathfrak{t}}^{+}=\left\{\bar{\alpha}_{p}, \bar{\alpha}_{p+q}, \bar{\alpha}_{p}+\bar{\alpha}_{p+q}, 2 \bar{\alpha}_{p+q}, \bar{\alpha}_{p}+2 \bar{\alpha}_{p+q}, 2 \bar{\alpha}_{p}+2 \bar{\alpha}_{p+q}\right\}$ and according to Proposition 3.5 (1) we obtain the decomposition $\mathfrak{m}=\mathfrak{m}_{1} \oplus \mathfrak{m}_{2} \oplus \mathfrak{m}_{3} \oplus \mathfrak{m}_{4} \oplus \mathfrak{m}_{5} \oplus \mathfrak{m}_{6}=$ $\mathfrak{m}(1,0) \oplus \mathfrak{m}(0,1) \oplus \mathfrak{m}(1,1) \oplus \mathfrak{m}(0,2) \oplus \mathfrak{m}(1,2) \oplus \mathfrak{m}(2,2)$. Note that the exception $p+q=\ell$ determines the space $M=\mathrm{Sp}(\ell) /(\mathrm{U}(p) \times \mathrm{U}(q))$ with four isotropy summands, since in this case the $\mathfrak{t}$-roots are given by $\Delta_{\mathrm{t}}^{+}=\left\{\bar{\alpha}_{p}, \bar{\alpha}_{\ell}, \bar{\alpha}_{p}+\bar{\alpha}_{\ell}, 2 \bar{\alpha}_{p}+\bar{\alpha}_{\ell}\right\}([\mathrm{ACh} 3])$.

\section{FlaG MANifoldS With Five isotropy SummnadS}

4.1. On the isotropy represantation of flag manifolds. Proposition 3.5 provides all the necessary ingredients for the classification of flag manifolds with a certain number of isotropy summands. However, we essentially need to work on a case by case basis, which means that in the Dynkin diagram $\Gamma(\Pi)$ of each simple Lie group $G$ we need to paint black all possible combinations of roots of certain Dynkin marks. A systematic approach for flag manifolds determined by a classical Lie group is given in [Ale2, but be aware of certain misprints. Recall that isotropy irreducible flag manifolds are the isotropy irreducible Hermitian symmetric spaces of compact type, and are determined by painting black exactly one simple root of Dynkin mark 1. Flag manifolds with two isotropy summnads were classified in [ACh1]. These spaces are determined by pairs $\left(\Pi, \Pi_{0}\right)$ such that $\Pi \backslash \Pi_{0}=\left\{\alpha_{p}: \operatorname{Mrk}\left(\alpha_{p}\right)=2\right\}$. Flag manifolds with three isotropy summands were classified in $\left[\mathrm{Kim}\right.$, where it was shown that such spaces are defined by pairs $\left(\Pi, \Pi_{0}\right)$ with that $\Pi \backslash \Pi_{0}=\left\{\alpha_{p}\right.$ : $\left.\operatorname{Mrk}\left(\alpha_{p}\right)=3\right\}$ or $\Pi \backslash \Pi_{0}=\left\{\alpha_{i}, \alpha_{j}: \operatorname{Mrk}\left(\alpha_{i}\right)=\operatorname{Mrk}\left(\alpha_{j}\right)=1\right\}$. Finally, the classification of all flag manifolds with four isotropy summands was given in ACh3, where it was shown that such spaces are determined by pairs $\left(\Pi, \Pi_{0}\right)$ such that $\Pi \backslash \Pi_{0}=\left\{\alpha_{p}: \operatorname{Mrk}\left(\alpha_{p}\right)=4\right\}$ or $\Pi \backslash \Pi_{0}=\left\{\alpha_{i}, \alpha_{j}: \operatorname{Mrk}\left(\alpha_{i}\right)=1, \operatorname{Mrk}\left(\alpha_{j}\right)=2\right\}$ (however the correpsondence with the second type of pairs is not one-to-one, see the Introduction).

In this section we will prove that the only generalized flag manifolds $G / K$ (different from the space $\left.\mathrm{E}_{8} / \mathrm{U}(1) \times \mathrm{SU}(4) \times \mathrm{SU}(5)\right)$, whose isotropy representation decomposes into five isotropy summands are the spaces determined by the pairs $\left(\Pi, \Pi_{0}\right)$ of Types $A$ and B presented in Table 1 in the Introduction. Furthermore, we will show that these pairs define isometric flag manifolds (as real manifolds), in the sense that there is an isometry which permutes the associated isotropy summands and identifies the different reductive decompositions (which are defined by the different pairs $\left(\Pi, \Pi_{0}\right)$ ). Therefore, our study focuces at the spaces listed in Table 2. This isometry (which is induced by the action of the associated Weyl group on the root system of $G$ ) enables us to study the classification problem of homogeneous Einstein metrics only for one possible pair $\left(\Pi, \Pi_{0}\right)$, therefore we will only work with flag manifolds of Type A. 
4.2. The classification of flag manifolds with five isotropy summnads. As mentioned in the Introduction, all flag manifolds of Types A and B are such that $b_{2}(M)=2$, which means that $\Pi_{\mathfrak{m}}=\Pi \backslash \Pi_{0}$ contains only two simple roots. For convenience of the reader in Table 3 we present all possible pairs $\left(\Pi, \Pi_{0}\right)$ which determine flag manifolds with $b_{2}(M)=2$ (for completeness we also include those which determine flag manifolds with $b_{2}(M)=1$ ). In this table, the first column contains the first Betti number, the second column indicates the Dynkin marks of the roots painted black, the third column shows the number $q$ of isotropy summands of the flag manifolds obtained (in some cases there are more than one possibilities), the fourth column shows for which Lie groups can arise such pairs (and thus such flag manifolds), and the last column gives references for the homogeneous Einstein metrics on the corresponding spaces.

Table 3. The isotropy representation and Einstein metrics on flag manifolds $M=G / K$ with $b_{2}(M)=1$ or 2

\begin{tabular}{|c|c|c|c|c|}
\hline$b_{2}(M)$ & Dynkin marks of $\Pi \backslash \Pi_{0}$ & $\mathfrak{m}=\bigoplus_{i=1}^{q} \mathfrak{m}_{q}$ & Type of $G$ & Einstein metrics \\
\hline 1 & $\operatorname{Mrk}\left(\alpha_{p}\right)=1$ & $q=1$ & Irred. Symmetric space & {$[$ Hel] } \\
\hline 1 & $\operatorname{Mrk}\left(\alpha_{p}\right)=2$ & $q=2$ & $B_{\ell}, C_{\ell}, D_{\ell}, \mathrm{G}_{2}, \mathrm{~F}_{4}, \mathrm{E}_{6}, \mathrm{E}_{7}, \mathrm{E}_{8}$ & ACh2 \\
\hline 1 & $\operatorname{Mrk}\left(\alpha_{p}\right)=3$ & $q=3$ & $\mathrm{G}_{2}, \mathrm{~F}_{4}, \mathrm{E}_{6}, \mathrm{E}_{7}, \mathrm{E}_{8}$ & $\mathrm{Kim}, \mathrm{AnCh}$ \\
\hline 1 & $\operatorname{Mrk}\left(\alpha_{p}\right)=4$ & $q=4$ & $\mathrm{~F}_{4}, \mathrm{E}_{7}, \mathrm{E}_{8}$ & ACh3 \\
\hline 1 & $\operatorname{Mrk}\left(\alpha_{p}\right)=5$ & $q=5$ & $\mathrm{E}_{8}$ & $\mathrm{ChSa}$ \\
\hline 1 & $\operatorname{Mrk}\left(\alpha_{p}\right)=6$ & $q=6$ & $\mathrm{E}_{8}$ & $\mathrm{ChSa}$, open \\
\hline 2 & $\operatorname{Mrk}\left(\alpha_{p}\right)=1, \operatorname{Mrk}\left(\alpha_{q}\right)=1$ & $q=3$ & $A_{\ell}, D_{\ell}, \mathrm{E}_{6}$ & Kim \\
\hline 2 & $\operatorname{Mrk}\left(\alpha_{p}\right)=1, \operatorname{Mrk}\left(\alpha_{q}\right)=2$ & $q=4,5$ & $B_{\ell}, C_{\ell}, D_{\ell}, \mathrm{E}_{6}, \mathrm{E}_{7}$ & ACh3, ACS1, ACS2 \\
\hline 2 & $\operatorname{Mrk}\left(\alpha_{p}\right)=1, \operatorname{Mrk}\left(\alpha_{q}\right)=3$ & $q=6$ & $\mathrm{E}_{6}, \mathrm{E}_{7}$ & open \\
\hline 2 & $\operatorname{Mrk}\left(\alpha_{p}\right)=1, \operatorname{Mrk}\left(\alpha_{q}\right)=4$ & $q=8$ & $\mathrm{E}_{7}$ & open \\
\hline 2 & $\operatorname{Mrk}\left(\alpha_{p}\right)=2, \operatorname{Mrk}\left(\alpha_{q}\right)=2$ & $q=5,6$ & $B_{\ell}, C_{\ell}, D_{\ell}, \mathrm{F}_{4}, \mathrm{E}_{6}, \mathrm{E}_{7}, \mathrm{E}_{8}$ & ACS3, Chr2, open \\
\hline 2 & $\operatorname{Mrk}\left(\alpha_{p}\right)=2, \operatorname{Mrk}\left(\alpha_{q}\right)=3$ & $q=6,7,8$ & $\mathrm{G}_{2}, \mathrm{~F}_{4}, \mathrm{E}_{6}, \mathrm{E}_{7}, \mathrm{E}_{8}$ & ACS4, open \\
\hline 2 & $\operatorname{Mrk}\left(\alpha_{p}\right)=2, \operatorname{Mrk}\left(\alpha_{q}\right)=4$ & $q=7,8,9$ & $\mathrm{~F}_{4}, \mathrm{E}_{7}, \mathrm{E}_{8}$ & open \\
\hline 2 & $\operatorname{Mrk}\left(\alpha_{p}\right)=2, \operatorname{Mrk}\left(\alpha_{q}\right)=5$ & $q=10$ & $\mathrm{E}_{8}$ & open \\
\hline 2 & $\operatorname{Mrk}\left(\alpha_{p}\right)=2, \operatorname{Mrk}\left(\alpha_{q}\right)=6$ & $q=11$ & $\mathrm{E}_{8}$ & open \\
\hline 2 & $\operatorname{Mrk}\left(\alpha_{p}\right)=3, \operatorname{Mrk}\left(\alpha_{q}\right)=3$ & $q=8,9$ & $\mathrm{E}_{7}, \mathrm{E}_{8}$ & open \\
\hline 2 & $\operatorname{Mrk}\left(\alpha_{p}\right)=3, \operatorname{Mrk}\left(\alpha_{q}\right)=4$ & $q=8,9,10$ & $\mathrm{~F}_{4}, \mathrm{E}_{7}, \mathrm{E}_{8}$ & open \\
\hline 2 & $\operatorname{Mrk}\left(\alpha_{p}\right)=3, \operatorname{Mrk}\left(\alpha_{q}\right)=5$ & $q=10,11$ & $\mathrm{E}_{8}$ & open \\
\hline 2 & $\operatorname{Mrk}\left(\alpha_{p}\right)=3, \operatorname{Mrk}\left(\alpha_{q}\right)=6$ & $q=10,14$ & $\mathrm{E}_{8}$ & open \\
\hline 2 & $\operatorname{Mrk}\left(\alpha_{p}\right)=4, \operatorname{Mrk}\left(\alpha_{q}\right)=4$ & $q=12$ & $\mathrm{E}_{8}$ & open \\
\hline 2 & $\operatorname{Mrk}\left(\alpha_{p}\right)=4, \operatorname{Mrk}\left(\alpha_{q}\right)=5$ & $q=10,11$ & $\mathrm{E}_{8}$ & open \\
\hline 2 & $\operatorname{Mrk}\left(\alpha_{p}\right)=4, \operatorname{Mrk}\left(\alpha_{q}\right)=6$ & $q=11,14$ & $\mathrm{E}_{8}$ & open \\
\hline 2 & $\operatorname{Mrk}\left(\alpha_{p}\right)=5, \operatorname{Mrk}\left(\alpha_{q}\right)=6$ & $q=12$ & $\mathrm{E}_{8}$ & open \\
\hline
\end{tabular}

In order to show that flag manifolds with five isotropy summnands (different from the space $\mathrm{E}_{8} / \mathrm{U}(1) \times$ $\mathrm{SU}(4) \times \mathrm{SU}(5)$ with $\left.b_{2}(M)=1\right)$ are determined only by the pairs $\left(\Pi, \Pi_{0}\right)$ presented in Table 1 of the Introduction, we proceed into two steps. First we show that the spaces determined by the pairs in Table 1 have in fact five isotropy summands. Next, we prove that the other existing pairs $\left(\Pi, \Pi_{0}\right)$ of Type B determine flag manifolds whose positive $\mathfrak{t}$-root system $\Delta_{\mathfrak{t}}^{+}$contains more than five elements. Note that pairs of Type A are excluded form the study due to [ACh3, Prop. 5] and the first step. All other pairs (П, $\left.\Pi_{0}\right)$ such that $\Pi \backslash \Pi_{0}=\left\{\alpha_{i}, \alpha_{j}\right\}$ and different from Types A and B can be treated in a similar manner, so we refer to [ACh3, Prop. 6] for further details and Table 3 the final results.

We need the following useful

Lemma 4.1. Generalized flag manifolds $M=G / K$ with $b_{2}(M) \geq 3$ have more than five isotropy summands.

Proof. At first we consider the case of $b_{2}(M)=3$ and the simple Lie groups $A_{\ell}$. We assume that the subset $\Pi_{0} \subset \Pi$ is such that $\Pi_{\mathfrak{m}}=\Pi \backslash \Pi_{0}=\left\{\alpha_{i}, \alpha_{j}, \alpha_{k}\right\}$ where $i, j, k$ are different each other. Then $\mathfrak{t}$ is 3 -dimensional and a $\mathfrak{t}$-basis is given by $\Pi_{\mathfrak{t}}=\left\{\bar{\alpha}_{i}=\left.\alpha_{i}\right|_{\mathfrak{t}}, \bar{\alpha}_{j}=\left.\alpha_{j}\right|_{\mathfrak{t}}, \bar{\alpha}_{k}=\left.\alpha_{k}\right|_{\mathfrak{t}}\right\}$ with $\operatorname{Mrk}\left(\alpha_{i}\right)=\operatorname{Mrk}\left(\alpha_{j}\right)=\operatorname{Mrk}\left(\alpha_{k}\right)=1$. Let $\alpha=\sum_{p=1}^{\ell} c_{p} \alpha_{p} \in \Delta_{\mathfrak{m}}^{+}$be a positive complementary root. Then, by applying (17) we conclude that any positive $\mathfrak{t}$-root is given by $\kappa(\alpha)=c_{i} \bar{\alpha}_{i}+c_{j} \bar{\alpha}_{j}+c_{k} \bar{\alpha}_{k}$, where $0 \leq c_{i}, c_{j}, c_{k} \leq 1$ cannot be simultaneosuly equal to zero and we see that the system $\Delta_{\mathrm{t}}^{+}$consists of the $\mathrm{t}$-roots $\bar{\alpha}_{i}, \bar{\alpha}_{j}, \bar{\alpha}_{k}, \bar{\alpha}_{i}+\bar{\alpha}_{j}, \bar{\alpha}_{j}+\bar{\alpha}_{k}, \bar{\alpha}_{i}+\bar{\alpha}_{j}+\bar{\alpha}_{k}$. 
Thus $\left|\Delta_{\mathfrak{t}}^{+}\right|=6$ and $\mathfrak{m}=T_{o} M$ decomposes into more than five isotropy summands. If $b_{2}(M)>3$, then the system $\Delta_{\mathrm{t}}^{+}$contains the $\mathrm{t}$-roots of the form $\bar{\alpha}_{i}, \bar{\alpha}_{j}, \bar{\alpha}_{k}, \bar{\alpha}_{i}+\bar{\alpha}_{j}, \bar{\alpha}_{j}+\bar{\alpha}_{k}, \bar{\alpha}_{i}+\bar{\alpha}_{j}+\bar{\alpha}_{k}$, and hence $\left|\Delta_{\mathrm{t}}^{+}\right|>5$. Now consider the case when the Dynkin diagram of a simple Lie algebra contains the Dynkin subdiagram of type $A_{m}$ and it contains these roots $\left\{\alpha_{i}, \alpha_{j}, \alpha_{k}\right\}$. Then we find that $\left|\Delta_{\mathfrak{t}}^{+}\right|>5$. The other cases are $D_{\ell}$ with $\left\{\alpha_{i}, \alpha_{\ell-1}, \alpha_{\ell}\right\}, \mathrm{E}_{6}, \mathrm{E}_{7}$ and $\mathrm{E}_{8}$. But for the case of $D_{\ell}$ with $\left\{\alpha_{i}, \alpha_{\ell-1}, \alpha_{\ell}\right\}$ we see that $\left|\Delta_{\mathfrak{t}}^{+}\right|>5$. If $\mathrm{E}_{6}, \mathrm{E}_{7}$ or $\mathrm{E}_{8}$ contains the Dynkin subdiagram of type $D_{m}$ which contains these roots $\left\{\alpha_{i}, \alpha_{m-1}, \alpha_{m}\right\}$, then it follows that $\left|\Delta_{\mathfrak{t}}^{+}\right|>5$. For the case $\mathrm{E}_{6}$ with $\left\{\alpha_{1}, \alpha_{5}, \alpha_{6}\right\}$ we get also $\left|\Delta_{\mathfrak{t}}^{+}\right|>5$. If $\mathrm{E}_{7}$ or $\mathrm{E}_{8}$ contains the Dynkin subdiagram of type $\mathrm{E}_{6}$ which contains these roots $\left\{\alpha_{i}, \alpha_{j}, \alpha_{k}\right\}$, then we see that $\left|\Delta_{\mathfrak{t}}^{+}\right|>5$. The remaining cases are $\mathrm{E}_{7}$ with $\left\{\alpha_{1}, \alpha_{6}, \alpha_{7}\right\}$ and $\mathrm{E}_{8}$ with $\left\{\alpha_{i}, \alpha_{7}, \alpha_{8}\right\}$ where $i=1,2,3$. For these cases one can easily prove that $\left|\Delta_{\mathfrak{t}}^{+}\right|>5$.

Thus flag manifolds with five isotropy summands are determined by pairs $\left(\Pi, \Pi_{0}\right)$ with $\left|\Pi \backslash \Pi_{0}\right|=2$.

Proposition 4.2. Let $G$ be a compact connected simple Lie group and let $\Pi=\left\{\alpha_{1}, \ldots, \alpha_{\ell}\right\}$ be a system of simple roots of the associated root system of $G$. Consider a subset $\Pi_{0} \subset \Pi$ of simple roots, such that $\Pi \backslash \Pi_{0}$ contains exactly two simple roots. Then, the only pairs $\left(\Pi, \Pi_{0}\right)$ which determine flag manifolds $G / K$ whose isotropy representation decomposes into five pairwise inquivalent irreducible $\operatorname{Ad}(K)$-submodules are the pairs of Type $A$ and $B$ presented in Table 1 of the Introduction.

Proof. Step 1. We follow the notation of [AlAr] or [ACh3] (see also [GOV]) for the root systems of the simple Lie algebras, their fundamental systems of simple roots and the associated expressions of the highest $\operatorname{root} \tilde{\alpha}$,

Case of $B_{\ell}=\mathrm{SO}(2 \ell+1)$ : Type A. Let $\Pi \backslash \Pi_{0}=\Pi_{\mathfrak{n}}=\left\{\alpha_{1}, \alpha_{p+1}: 2 \leq p \leq \ell-1\right\}$. This choice corresponds to the painted Dynkin diagram

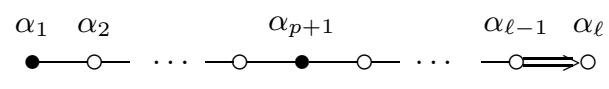

which determines the flag manifold $M=\mathrm{SO}(2 \ell+1) /(\mathrm{U}(1) \times \mathrm{U}(p) \times \mathrm{SO}(2(\ell-p-1)+1))$ with $2 \leq p \leq \ell-1$ and $\ell \geq 3$. Let $\mathfrak{n}$ be a $B$-orthogonal complement of the isotropy subalgebra $\mathfrak{k}$ in $\mathfrak{g}=\mathfrak{s o}(2 \ell+1)$, that is $\mathfrak{g}=\mathfrak{k} \oplus \mathfrak{n}$ with $[\mathfrak{k}, \mathfrak{n}] \subset \mathfrak{n}$. We will prove that the $\operatorname{Ad}(K)$-module $\mathfrak{n} \cong T_{o} M$ decomposes into a direct sum of five non equivalent $\operatorname{Ad}(K)$ - submodules $\mathfrak{n}_{i}(i=1, \ldots, 5)$ whose dimensions are given by (21).

Let $\left\{e_{1}^{1}, e_{i}^{2}, \pi_{j}\right\}$ be an orthonormal basis of $\mathbb{R}^{\ell}$ with $1 \leq i \leq p$ and $1 \leq j \leq \ell-p-1$. The positive root system $\Delta^{+}$of $\mathrm{SO}(2 \ell+1)$ is given by (see $[\mathrm{AlAr}$ )

$$
\begin{aligned}
\Delta^{+}= & \left\{e_{1}^{1} \pm e_{i}^{2}: 1 \leq i \leq p\right\} \cup\left\{e_{i}^{2} \pm e_{j}^{2}: 1 \leq i<j \leq p\right\} \cup\left\{e_{1}^{1} \pm \pi_{j}: 1 \leq j \leq \ell-p-1\right\} \\
& \cup\left\{e_{i}^{2} \pm \pi_{j},: 1 \leq i \leq p, 1 \leq j \leq \ell-p-1\right\} \cup\left\{\pi_{i} \pm \pi_{j}: 1 \leq i<j \leq \ell-p-1\right\} \\
& \cup\left\{e_{1}^{1}, e_{i}^{2}: 1 \leq i \leq p\right\} \cup\left\{\pi_{j}: 1 \leq j \leq \ell-p-1\right\}
\end{aligned}
$$

We will denote a basis of simple roots by

$$
\begin{array}{r}
\Pi(\mathfrak{n})=\left\{\alpha_{1}=e_{1}^{1}-e_{2}^{1}, \alpha_{2}=e_{1}^{2}-e_{2}^{2}, \ldots, \alpha_{p}=e_{p-1}^{2}-e_{p}^{2}, \alpha_{p+1}=e_{p}^{2}-\pi_{1},\right. \\
\left.\phi_{1}=\pi_{1}-\pi_{2}, \ldots, \phi_{\ell-p-2}=\pi_{\ell-p-2}-\pi_{\ell-p-1}, \phi_{\ell-p-1}=\pi_{\ell-p-1}\right\} .
\end{array}
$$

It is $\Delta_{o}^{+}=\left\{e_{i}^{2}-e_{j}^{2}, \pi_{i} \pm \pi_{j}, \pi_{j}\right\}$ and thus the positive complementary roots are given by $\Delta_{\mathfrak{n}}^{+}=\left\{e_{1}^{1} \pm e_{i}^{2}, e_{i}^{2}+\right.$ $\left.e_{j}^{2}, e_{1}^{1} \pm \pi_{j}, e_{i}^{2} \pm \pi_{j}, e_{1}^{1}, e_{i}^{2}\right\}$. According to (17) for any $\alpha=\sum_{k=1}^{\ell} c_{k} \alpha_{k} \in \Delta_{\mathfrak{n}}^{+}$it will be $\kappa(\alpha)=c_{1} \bar{\alpha}_{1}+c_{p+1} \bar{\alpha}_{p+1}$ with $0 \leq c_{1} \leq 1$ and $0 \leq c_{p+1} \leq 2$. In particular, by expressing the complementary roots in terms of simple 
roots we obtain that

$$
\begin{aligned}
\kappa\left(e_{1}^{1}-e_{i}^{2}\right) & =\kappa\left(\alpha_{1}+\alpha_{2}+\cdots+\alpha_{i}\right)=\bar{\alpha}_{1}, \\
\kappa\left(e_{1}^{1}+e_{i}^{2}\right) & =\kappa\left(\alpha_{1}+\cdots+\alpha_{i}+2 \alpha_{i+1}+\cdots 2 \alpha_{p+1}+2 \phi_{1}+\cdots+2 \phi_{\ell-p-1}\right)=\bar{\alpha}_{1}+2 \bar{\alpha}_{p+1}, \\
\kappa\left(e_{i}^{2}+e_{j}^{2}\right) & =\kappa\left(\alpha_{i+1}+\cdots \alpha_{j}+2 \alpha_{j+1}+\cdots 2 \alpha_{p+1}+2 \phi_{1}+\cdots+2 \phi_{\ell-p-1}\right)=2 \bar{\alpha}_{p+1}, \\
\kappa\left(e_{1}^{1}-\pi_{j}\right) & =\kappa\left(\alpha_{1}+\cdots+\alpha_{p+1}+\phi_{1}+\cdots \phi_{j-1}\right)=\bar{\alpha}_{1}+\bar{\alpha}_{p+1} \\
\kappa\left(e_{1}^{1}+\pi_{j}\right) & =\kappa\left(\alpha_{1}+\cdots+\alpha_{p+1}+\phi_{1}+\cdots+\phi_{j-1}+2 \phi_{j}+2 \phi_{j+1}+\cdots+2 \phi_{\ell-p-1}\right)=\bar{\alpha}_{1}+\bar{\alpha}_{p+1}, \\
\kappa\left(e_{i}^{2}+\pi_{j}\right) & =\kappa\left(\alpha_{i+1}+\cdots+\alpha_{p+1}+\phi_{1}+\cdots+\phi_{j-1}+2 \phi_{j}+\cdots+2 \phi_{\ell-p-1}\right)=\bar{\alpha}_{p+1}, \\
\kappa\left(e_{i}^{2}-\pi_{j}\right) & =\kappa\left(\alpha_{i+1}+\cdots+\alpha_{p+1}+\phi_{1}+\cdots+\phi_{j-1}\right)=\bar{\alpha}_{p+1}, \\
\kappa\left(e_{1}^{1}\right) & =\kappa\left(\alpha_{1}+\cdots+\phi_{\ell-p-1}\right)=\bar{\alpha}_{1}+\bar{\alpha}_{p+1}, \\
\kappa\left(e_{i}^{2}\right) & =\kappa\left(\alpha_{i+1}+\cdots+\alpha_{p+1}+\phi_{1}+\cdots+\phi_{\ell-p-1}\right)=\bar{\alpha}_{p+1} .
\end{aligned}
$$

Let $\Delta(\mathfrak{n})_{\mathfrak{t}}^{+}$be the associated system of positive $\mathfrak{t}$-roots. Then it follows that $\Delta(\mathfrak{n})_{\mathfrak{t}}^{+}=\left\{\bar{\alpha}_{1}, \bar{\alpha}_{p+1}, \bar{\alpha}_{1}+\right.$ $\left.\bar{\alpha}_{p+1}, 2 \bar{\alpha}_{p+1}, \bar{\alpha}_{1}+2 \bar{\alpha}_{p+1}\right\}$, and thus according to Proposition 3.5 (1) we obtain the decomposition

$$
\mathfrak{n}=\mathfrak{n}_{1} \oplus \mathfrak{n}_{2} \oplus \mathfrak{n}_{3} \oplus \mathfrak{n}_{4} \oplus \mathfrak{n}_{5}=\mathfrak{n}(1,0) \oplus \mathfrak{n}(0,1) \oplus \mathfrak{n}(1,1) \oplus \mathfrak{n}(0,2) \oplus \mathfrak{n}(1,2)
$$

where the submodules $\mathfrak{n}_{i}$ are given by

$$
\begin{aligned}
& \mathfrak{n}_{1}=\mathfrak{n}(1,0)=\sum_{\alpha \in \Delta^{\mathfrak{n}}(1,0)}\left\{\mathbb{R} A_{\alpha}+\mathbb{R} B_{\alpha}\right\}=\sum_{\alpha \in \Delta_{\mathfrak{n}}^{+}: \kappa(\alpha)=\bar{\alpha}_{1}}\left\{\mathbb{R} A_{\alpha}+\mathbb{R} B_{\alpha}\right\} \\
& \mathfrak{n}_{2}=\mathfrak{n}(0,1)=\sum_{\alpha \in \Delta^{\mathfrak{n}}(0,1)}\left\{\mathbb{R} A_{\alpha}+\mathbb{R} B_{\alpha}\right\}=\sum_{\alpha \in \Delta_{\mathfrak{n}}^{+}: \kappa(\alpha)=\bar{\alpha}_{p+1}}\left\{\mathbb{R} A_{\alpha}+\mathbb{R} B_{\alpha}\right\} \\
& \mathfrak{n}_{3}=\mathfrak{n}(1,1)=\sum_{\alpha \in \Delta^{\mathfrak{n}}(1,1)}\left\{\mathbb{R} A_{\alpha}+\mathbb{R} B_{\alpha}\right\}=\sum_{\alpha \in \Delta_{\mathfrak{n}}^{+}:} \sum_{\kappa(\alpha)=\bar{\alpha}_{1}+\bar{\alpha}_{p+1}}\left\{\mathbb{R} A_{\alpha}+\mathbb{R} B_{\alpha}\right\} \\
& \mathfrak{n}_{4}=\mathfrak{n}(0,2)=\sum_{\alpha \in \Delta^{\mathfrak{n}}(0,2)}\left\{\mathbb{R} A_{\alpha}+\mathbb{R} B_{\alpha}\right\}=\sum_{\alpha \in \Delta_{\mathfrak{n}}^{+}: \kappa(\alpha)=2 \bar{\alpha}_{p+1}}\left\{\mathbb{R} A_{\alpha}+\mathbb{R} B_{\alpha}\right\} \\
& \mathfrak{n}_{5}=\mathfrak{n}(1,2)=\sum_{\alpha \in \Delta^{\mathfrak{n}}(1,2)}\left\{\mathbb{R} A_{\alpha}+\mathbb{R} B_{\alpha}\right\}=\sum_{\alpha \in \Delta_{\mathfrak{n}}^{+}: \kappa(\alpha)=\bar{\alpha}_{1}+2 \bar{\alpha}_{p+1}}\left\{\mathbb{R} A_{\alpha}+\mathbb{R} B_{\alpha}\right\}
\end{aligned}
$$

The sets $\Delta^{\mathfrak{n}}\left(j_{1}, j_{2}\right)$ are given by

$$
\begin{aligned}
\Delta^{\mathfrak{n}}(1,0) & =\left\{e_{1}^{1}-e_{i}^{2}: 1 \leq i \leq p\right\} \\
\Delta^{\mathfrak{n}}(0,1) & =\left\{e_{i}^{2} \pm \pi_{j}: 1 \leq i \leq p, 1 \leq j \leq \ell-p-1\right\} \cup\left\{e_{i}^{2}: 1 \leq i \leq p\right\} \\
\Delta^{\mathfrak{n}}(1,1) & =\left\{e_{1}^{1} \pm \pi_{j}, e_{1}^{1}: 1 \leq j \leq \ell-p-1\right\} \\
\Delta^{\mathfrak{n}}(0,2) & =\left\{e_{i}^{2}+e_{j}^{2}: 1 \leq i<j \leq p\right\} \\
\Delta^{\mathfrak{n}}(1,2) & =\left\{e_{1}^{1}+e_{i}^{2}: 1 \leq i \leq p\right\}
\end{aligned}
$$

Thus by applying Proposition 3.5 (2), we conclude that the dimensions of these submodules are given as follows:

$$
\begin{aligned}
& \operatorname{dim}_{\mathbb{R}} \mathfrak{n}_{1}=2 \cdot\left|\left\{\alpha \in \Delta_{\mathfrak{n}}^{+}: \kappa(\alpha)=\bar{\alpha}_{1}\right\}\right|=2 \cdot\left|\Delta^{\mathfrak{n}}(1,0)\right|=2 p \\
& \operatorname{dim}_{\mathbb{R}} \mathfrak{n}_{2}=2 \cdot\left|\left\{\alpha \in \Delta_{\mathfrak{n}}^{+}: \kappa(\alpha)=\bar{\alpha}_{p+1}\right\}\right|=2 \cdot\left|\Delta^{\mathfrak{n}}(0,1)\right|=2 p(2 \ell-2 p-1), \\
& \operatorname{dim}_{\mathbb{R}} \mathfrak{n}_{3}=2 \cdot\left|\left\{\alpha \in \Delta_{\mathfrak{n}}^{+}: \kappa(\alpha)=\bar{\alpha}_{1}+\bar{\alpha}_{p+1}\right\}\right|=2 \cdot\left|\Delta^{\mathfrak{n}}(1,1)\right|=2(2 \ell-2 p-1), \\
& \operatorname{dim}_{\mathbb{R}} \mathfrak{n}_{4}=2 \cdot\left|\left\{\alpha \in \Delta_{\mathfrak{n}}^{+}: \kappa(\alpha)=2 \bar{\alpha}_{p+1}\right\}\right|=2 \cdot\left|\Delta^{\mathfrak{n}}(0,2)\right|=p(p-1), \\
& \operatorname{dim}_{\mathbb{R}} \mathfrak{n}_{5}=2 \cdot\left|\left\{\alpha \in \Delta_{\mathfrak{n}}^{+}: \kappa(\alpha)=\bar{\alpha}_{1}+2 \bar{\alpha}_{p+1}\right\}\right|=2 \cdot\left|\Delta^{\mathfrak{n}}(1,2)\right|=2 p
\end{aligned}
$$

Note that for $p=\ell-1$, that is $\Pi_{\mathfrak{n}}=\left\{\alpha_{1}, \alpha_{\ell}\right\}$, we have the following painted Dynkin diagram

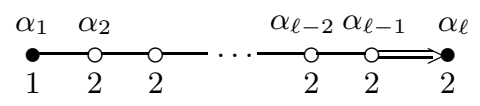

The corresponding flag manifold $M=\mathrm{SO}(2 \ell+1) / \mathrm{U}(1) \times \mathrm{U}(\ell-1)(\ell \geq 3)$ has also five isotropy summands. However, note that the relation $\left(\alpha_{1}, \alpha_{1}\right)=\left(\alpha_{p+1}, \alpha_{p+1}\right)(3 \leq p \leq \ell-2)$ is no longer true. It means that for the case $p=\ell-1$ the painted black simple roots have different lengths.

Case of $B_{\ell}=\mathrm{SO}(2 \ell+1)$ : Type B. We now assume that the pair $\left(\Pi, \Pi_{0}\right)$ is of Type $\mathrm{B}$, that is $\Pi \backslash \Pi_{0}=$ $\Pi_{\mathfrak{m}}=\left\{\alpha_{p}, \alpha_{p+1}: 2 \leq p \leq \ell-1\right\}$. This choice corrsponds to the following painted Dynkin diagram: 


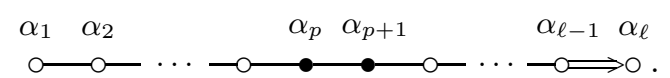

which also defines the flag manifold $M=\mathrm{SO}(2 \ell+1) /(\mathrm{U}(1) \times \mathrm{U}(p) \times \mathrm{SO}(2(\ell-p-1)+1))$ with $2 \leq p \leq \ell-1$ and $\ell \geq 3$. Let $\mathfrak{g}=\mathfrak{k} \oplus \mathfrak{m}$ be a reductive decomposition of $\mathfrak{g}=\mathfrak{s o}(2 \ell+1)$, with respect to $B$. Similarly with Type $\mathrm{A}$, we consider an orthonormal basis of $\mathbb{R}^{\ell}$ given by $\left\{e_{i}^{1}, e_{1}^{2}, \pi_{j}\right\}$ with $1 \leq i \leq p$ and $1 \leq j \leq \ell-p-1$. Then, a system of positive roots is given by

$$
\begin{aligned}
\Delta^{+}= & \left\{e_{i}^{1} \pm e_{j}^{1}: 1 \leq i<j \leq p\right\} \cup\left\{e_{i}^{1} \pm e_{1}^{2}: 1 \leq i \leq p\right\} \cup\left\{e_{i}^{1} \pm \pi_{j}: 1 \leq i \leq p, 1 \leq j \leq \ell-p-1\right\} \\
& \cup\left\{e_{1}^{2} \pm \pi_{j}: 1 \leq j \leq \ell-p-1\right\} \cup\left\{\pi_{i} \pm \pi_{j}: 1 \leq i<j \leq \ell-p-1\right\} \\
& \cup\left\{e_{1}^{2}, e_{i}^{1}: 1 \leq i \leq p\right\} \cup\left\{\pi_{j}: 1 \leq j \leq \ell-p-1\right\} .
\end{aligned}
$$

In this case, we denote a base of simple roots by

$$
\begin{gathered}
\Pi(\mathfrak{m})=\left\{\alpha_{1}=e_{1}^{1}-e_{2}^{1}, \alpha_{2}=e_{2}^{1}-e_{3}^{1}, \ldots, \alpha_{p-1}=e_{p-1}^{1}-e_{p}^{1}, \alpha_{p}=e_{p}^{1}-e_{1}^{2}, \alpha_{p+1}=e_{1}^{2}-\pi_{1},\right. \\
\left.\phi_{1}=\pi_{1}-\pi_{2}, \ldots, \phi_{\ell-p-2}=\pi_{\ell-p-2}-\pi_{\ell-p-1}, \phi_{\ell-p-1}=\pi_{\ell-p-1}\right\} .
\end{gathered}
$$

The root system of the semisimple part of the isotropy subgroup $K$ is given by $\Delta_{0}^{+}=\left\{e_{i}^{1}-e_{j}^{1}, \pi_{i} \pm \pi_{j}, \pi_{j}\right.$ : $i<j\}$ and thus the positive complementary roots are of the form

$$
\Delta_{\mathfrak{m}}^{+}=\left\{e_{i}^{1}+e_{j}^{1}, e_{i}^{1} \pm e_{1}^{2}, e_{i}^{1} \pm \pi_{j}, e_{1}^{2} \pm \pi_{j}, e_{i}^{1}, e_{1}^{2}: i<j\right\} .
$$

Choose $\alpha=\sum_{k=1}^{\ell} c_{k} \alpha_{k} \in \Delta_{\mathfrak{m}}^{+}$. Since $\Pi_{\mathfrak{m}}=\left\{\alpha_{p}, \alpha_{p+1}\right\}$, by applying relation (17) we obtain that $\kappa(\alpha)=$ $c_{p} \bar{\alpha}_{p}+c_{p+1} \bar{\alpha}_{p+1} \in \Delta(\mathfrak{m})_{\mathfrak{t}}^{+}$where $\Delta(\mathfrak{m})_{\mathfrak{t}}^{+}$is the associated system of positive $\mathfrak{t}$-roots. Here the coefficients $c_{p}, c_{p+1}$ are such that $0 \leq c_{p} \leq 2$, and $0 \leq c_{p+1} \leq 2$, and they are not simultaneously equal to zero. By expressing the complementary roots in terms of simple roots we conclude that $\Delta(\mathfrak{m})_{\mathfrak{t}}^{+}=\left\{\bar{\alpha}_{p}, \bar{\alpha}_{p+1}, \bar{\alpha}_{p}+\right.$ $\left.\bar{\alpha}_{p+1}, \bar{\alpha}_{p}+2 \bar{\alpha}_{p+1}, 2 \bar{\alpha}_{p}+2 \bar{\alpha}_{p+1}\right\}$. Indeed, it is

$$
\begin{aligned}
\kappa\left(e_{i}^{1}-e_{1}^{2}\right) & =\kappa\left(\alpha_{i}+\cdots+\alpha_{p}\right)=\bar{\alpha}_{p}, \\
\kappa\left(e_{i}^{1}+e_{1}^{2}\right) & =\kappa\left(\alpha_{i}+\cdots+\alpha_{p}+2 \alpha_{p+1}+2 \phi_{1}+\cdots+\cdots+2 \phi_{\ell-p-1}\right)=\bar{\alpha}_{p}+2 \bar{\alpha}_{p+1}, \\
\kappa\left(e_{i}^{1}+e_{j}^{1}\right) & =\kappa\left(\alpha_{i}+\cdots+2 \alpha_{j}+\cdots+2 \alpha_{p}+2 \alpha_{p+1}+2 \phi_{1}+\cdots+2 \phi_{\ell-p-1}\right)=2 \bar{\alpha}_{p}+2 \bar{\alpha}_{p+1}, \\
\kappa\left(e_{i}^{1}-\pi_{j}\right) & =\kappa\left(\alpha_{i}+\cdots+\alpha_{p}+\alpha_{p+1}+\phi_{1}+\cdots+\phi_{j}\right)=\bar{\alpha}_{p}+\bar{\alpha}_{p+1} \\
\kappa\left(e_{i}^{1}+\pi_{j}\right) & =\kappa\left(\alpha_{i}+\cdots+\alpha_{p}+\alpha_{p+1}+\phi_{1}+\cdots+\phi_{j}+2 \phi_{j+1}+\cdots+2 \phi_{\ell-p-1}\right)=\bar{\alpha}_{p}+\bar{\alpha}_{p+1}, \\
\kappa\left(e_{1}^{2}-\pi_{j}\right) & =\kappa\left(\alpha_{p+1}+\phi_{1}+\cdots+\phi_{j}\right)=\bar{\alpha}_{p+1}, \\
\kappa\left(e_{1}^{2}+\pi_{j}\right) & =\kappa\left(\alpha_{p+1}+\phi_{1}+\cdots+\phi_{j}+2 \phi_{j+1}+\cdots+2 \phi_{\ell-p-1}\right)=\bar{\alpha}_{p+1}, \\
\kappa\left(e_{1}^{2}\right) & =\kappa\left(\alpha_{p+1}+\phi_{1}+\cdots+\phi_{\ell-p-1}\right)=\bar{\alpha}_{p+1}, \\
\kappa\left(e_{i}^{1}\right) & =\kappa\left(\alpha_{i}+\cdots+\alpha_{p}+\alpha_{p+1}+\phi_{1}+\cdots+\phi_{\ell-p-1}\right)=\bar{\alpha}_{p}+\bar{\alpha}_{p+1} .
\end{aligned}
$$

Thus, by applying Proposition 3.5 (1) we conclude that the associated isotropy representation decomposes into a direct sum of five isotropy summands, i.e.

$$
\mathfrak{m}=\mathfrak{m}_{1} \oplus \mathfrak{m}_{2} \oplus \mathfrak{m}_{3} \oplus \mathfrak{m}_{4} \oplus \mathfrak{m}_{5}=\mathfrak{m}(1,0) \oplus \mathfrak{m}(0,1) \oplus \mathfrak{m}(1,1) \oplus \mathfrak{m}(1,2) \oplus \mathfrak{m}(2,2)
$$

By applying Proposition 3.5 (2) we obtain that

$$
\begin{aligned}
& \operatorname{dim}_{\mathbb{R}} \mathfrak{m}_{1}=2 \cdot\left|\left\{\alpha \in \Delta_{\mathfrak{m}}^{+}: \kappa(\alpha)=\bar{\alpha}_{p}\right\}\right|=2 \cdot\left|\Delta^{\mathfrak{m}}(1,0)\right|=2 p \\
& \operatorname{dim}_{\mathbb{R}} \mathfrak{m}_{2}=2 \cdot\left|\left\{\alpha \in \Delta_{\mathfrak{m}}^{+}: \kappa(\alpha)=\bar{\alpha}_{p+1}\right\}\right|=2 \cdot\left|\Delta^{\mathfrak{m}}(0,1)\right|=2(2 \ell-2 p-1), \\
& \operatorname{dim}_{\mathbb{R}} \mathfrak{m}_{3}=2 \cdot\left|\left\{\alpha \in \Delta_{\mathfrak{m}}^{+}: \kappa(\alpha)=\bar{\alpha}_{p}+\bar{\alpha}_{p+1}\right\}\right|=2 \cdot\left|\Delta^{\mathfrak{m}}(1,1)\right|=2 p(2 \ell-2 p-1), \\
& \operatorname{dim}_{\mathbb{R}} \mathfrak{m}_{4}=2 \cdot\left|\left\{\alpha \in \Delta_{\mathfrak{m}}^{+}: \kappa(\alpha)=\bar{\alpha}_{p}+2 \bar{\alpha}_{p+1}\right\}\right|=2 \cdot\left|\Delta^{\mathfrak{m}}(1,2)\right|=2 p \\
& \operatorname{dim}_{\mathbb{R}} \mathfrak{m}_{5}=2 \cdot\left|\left\{\alpha \in \Delta_{\mathfrak{m}}^{+}: \kappa(\alpha)=2 \bar{\alpha}_{p}+2 \bar{\alpha}_{p+1}\right\}\right|=2 \cdot\left|\Delta^{\mathfrak{m}}(2,2)\right|=p(p-1)
\end{aligned}
$$

Note that for $p=1$ the space $M=\mathrm{SO}(2 \ell+1) /(\mathrm{U}(1) \times \mathrm{U}(1) \times \mathrm{SO}(2(\ell-2)+1))$ has four isotropy summands (ACh3]). On the other hand, the case $p=\ell-1$ corresponds to the painted Dynkin diagram $\Gamma\left(\Pi_{\mathfrak{m}}\right)$ with $\Pi_{\mathfrak{m}}=\left\{\alpha_{\ell-1}, \alpha_{\ell}\right\}(\ell \geq 3)$, that is

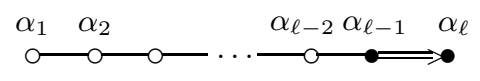


The corresponding flag manifold $M=\mathrm{SO}(2 \ell+1) / \mathrm{U}(\ell-1) \times \mathrm{U}(1)(\ell \geq 3)$ satisfies decomposition (22) as well, but in this case the painted black roots are such that $\left(\alpha_{\ell-1}, \alpha_{\ell-1}\right)=2\left(\alpha_{\ell}, \alpha_{\ell}\right)$, i.e. they have different length.

Case of $D_{\ell}=\mathrm{SO}(2 \ell)$ : Type A. Let $\Pi \backslash \Pi_{0}=\Pi_{\mathfrak{n}}=\left\{\alpha_{1}, \alpha_{p+1}: 2 \leq p \leq \ell-3\right\}$. This choice corresponds to the painted Dynkin diagram

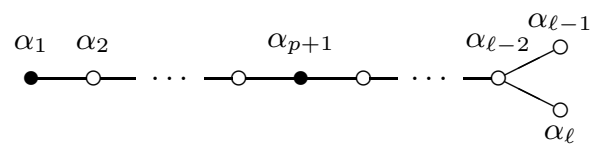

which determines the flag manifold $M=\mathrm{SO}(2 \ell) /(\mathrm{U}(1) \times \mathrm{U}(p) \times \mathrm{SO}(2(\ell-p-1)))$ with $2 \leq p \leq \ell-3$ and $\ell \geq 5$.

Similarly with the previous cases we obtain that the positive $\mathfrak{t}$-roots are given by $\Delta(\mathfrak{n})_{\mathfrak{t}}^{+}=\left\{\bar{\alpha}_{1}, \bar{\alpha}_{p+1}, \bar{\alpha}_{1}+\right.$ $\left.\bar{\alpha}_{p+1}, 2 \bar{\alpha}_{p+1}, \bar{\alpha}_{1}+2 \bar{\alpha}_{p+1}\right\}$ and according to Proposition 3.5 (1) we obtain the decomposition (18) where the submodules $\mathfrak{n}_{i}$ are determined by (19).

By applying Proposition 3.5 (2) we obtain the dimensions of $\mathfrak{n}_{i}$ :

$$
\left.\begin{array}{rl}
\operatorname{dim}_{\mathbb{R}} \mathfrak{n}_{1} & =2 \cdot\left|\left\{\alpha \in \Delta_{\mathfrak{m}}^{+}: \kappa(\alpha)=\bar{\alpha}_{1}\right\}\right|=2 p, \\
\operatorname{dim}_{\mathbb{R}} \mathfrak{n}_{2} & =2 \cdot\left|\left\{\alpha \in \Delta_{\mathfrak{m}}^{+}: \kappa(\alpha)=\bar{\alpha}_{p+1}\right\}\right|=4 p(\ell-p-1), \\
\operatorname{dim}_{\mathbb{R}} \mathfrak{n}_{3}=2 \cdot\left|\left\{\alpha \in \Delta_{\mathfrak{m}}^{+}: \kappa(\alpha)=\bar{\alpha}_{1}+\bar{\alpha}_{p+1}\right\}\right|=4(\ell-p-1), & \\
\operatorname{dim}_{\mathbb{R}} \mathfrak{n}_{4}=2 \cdot\left|\left\{\alpha \in \Delta_{\mathfrak{m}}^{+}: \kappa(\alpha)=2 \bar{\alpha}_{p+1}\right\}\right|=p(p-1), \\
\operatorname{dim}_{\mathbb{R}} \mathfrak{n}_{5}=2 \cdot\left|\left\{\alpha \in \Delta_{\mathfrak{m}}^{+}: \kappa(\alpha)=\bar{\alpha}_{1}+2 \bar{\alpha}_{p+1}\right\}\right|=2 p .
\end{array}\right\}
$$

Case of $D_{\ell}=\mathrm{SO}(2 \ell)$ : Type B. We now examine the pair $\left(\Pi, \Pi_{0}\right)$ of Type B corresponding to $D_{\ell}$, that is $\Pi \backslash \Pi_{0}=\Pi_{\mathfrak{m}}=\left\{\alpha_{p}, \alpha_{p+1}: 2 \leq p \leq \ell-3\right\}$. This choice corresponds to the painted Dynkin diagram

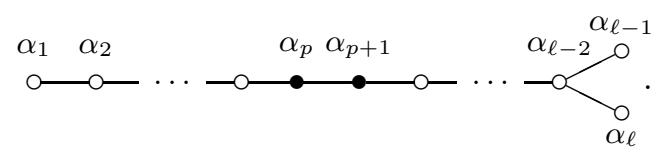

which also determines the flag manifold $M=\mathrm{SO}(2 \ell) /(\mathrm{U}(1) \times \mathrm{U}(p) \times \mathrm{SO}(2(\ell-p-1)))$, with $2 \leq p \leq \ell-3$ and $\ell \geq 5$. It follows that $\Delta(\mathfrak{m})_{\mathfrak{t}}^{+}=\left\{\bar{\alpha}_{p}, \bar{\alpha}_{p+1}, \bar{\alpha}_{p}+\bar{\alpha}_{p+1}, \bar{\alpha}_{p}+2 \bar{\alpha}_{p+1}, 2 \bar{\alpha}_{p}+2 \bar{\alpha}_{p+1}\right\}$, and by using Proposition 3.5 we conclude that the dimensions of these submodules are given as follows:

$$
\left.\begin{array}{l}
\operatorname{dim}_{\mathbb{R}} \mathfrak{m}_{1}=2 \cdot\left|\left\{\alpha \in \Delta_{\mathfrak{m}}^{+}: \kappa(\alpha)=\bar{\alpha}_{p}\right\}\right|=2 p, \\
\operatorname{dim}_{\mathbb{R}} \mathfrak{m}_{2}=2 \cdot\left|\left\{\alpha \in \Delta_{\mathfrak{m}}^{+}: \kappa(\alpha)=\bar{\alpha}_{p+1}\right\}\right|=4(\ell-p-1), \\
\operatorname{dim}_{\mathbb{R}} \mathfrak{m}_{3}=2 \cdot\left|\left\{\alpha \in \Delta_{\mathfrak{m}}^{+}: \kappa(\alpha)=\bar{\alpha}_{p}+\bar{\alpha}_{p+1}\right\}\right|=4 p(\ell-p-1), \\
\operatorname{dim}_{\mathbb{R}} \mathfrak{m}_{4}=2 \cdot\left|\left\{\alpha \in \Delta_{\mathfrak{m}}^{+}: \kappa(\alpha)=\bar{\alpha}_{p}+2 \bar{\alpha}_{p+1}\right\}\right|=2 p, \\
\operatorname{dim}_{\mathbb{R}} \mathfrak{m}_{5}=2 \cdot\left|\left\{\alpha \in \Delta_{\mathfrak{m}}^{+}: \kappa(\alpha)=2 \bar{\alpha}_{p}+2 \bar{\alpha}_{p+1}\right\}\right|=p(p-1) .
\end{array}\right\}
$$

Case of $\mathrm{E}_{6}:$ Type A. The highest root $\tilde{\alpha}$ of $\mathrm{E}_{6}$ is given by $\tilde{\alpha}=\alpha_{1}+2 \alpha_{2}+3 \alpha_{3}+2 \alpha_{4}+\alpha_{5}+2 \alpha_{6}$. Thus, for $\mathrm{E}_{6}$ we find two pairs $\left(\Pi, \Pi_{0}\right)$ of Type $\mathrm{A}$, which determine flag manifolds with five isotropy summands, namely the choices $\Pi \backslash \Pi_{0}=\left\{\alpha_{1}, \alpha_{4}\right\}$ and $\Pi \backslash \Pi_{0}=\left\{\alpha_{2}, \alpha_{5}\right\}$. They correspond to the painted Dynkin diagrams
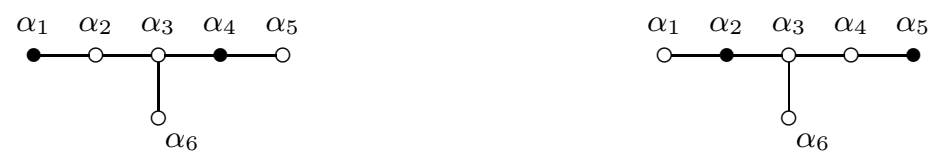

which both define the flag manifold $\mathrm{E}_{6} / \mathrm{SU}(4) \times \mathrm{SU}(2) \times \mathrm{U}(1)^{2}$. However, there is an outer automorphism of $\mathrm{E}_{6} \sqrt{4}$ which makes these painted Dynkin diagrams equivalent (see $\mathrm{BFR}$ ). Thus we will not distinguish these two pairs $\left(\Pi, \Pi_{0}\right)$ and we will work with the first one. Let $\mathfrak{n}$ be the $B$-orthogonal complement of the isotropy subalgebra $\mathfrak{k}$ in $\mathfrak{e}_{6}$. For the root system of $\mathrm{E}_{6}$ we use the notation of $[\mathrm{AlAr}$, where all positive roots

\footnotetext{
${ }^{4}$ The group of outer automorphisms of a simple Lie algebra is precisely the group of graph automorphisms of the associated Dynkin diagram. It is known that for the exceptional simple Lie algebras over $\mathbb{C}$, outer automorphisms exist only for $\mathrm{E}_{6}$.
} 
are given as linear combinations of the simple roots $\Pi=\left\{\alpha_{1}, \ldots, \alpha_{6}\right\}$. The root system of the semisimple part of the isotropy subgroup $K$ is given by $\Delta_{0}^{+}=\left\{\alpha_{2}, \alpha_{3}, \alpha_{5}, \alpha_{6}, \alpha_{2}+\alpha_{3}, \alpha_{3}+\alpha_{6}, \alpha_{2}+\alpha_{3}+\alpha_{6}\right\}$, thus

$$
\Delta_{\mathfrak{n}}^{+}=\left\{\begin{array}{lll}
\alpha_{1}+2 \alpha_{2}+3 \alpha_{3}+2 \alpha_{4}+\alpha_{5}+2 \alpha_{6} & \alpha_{1}+\alpha_{2}+\alpha_{3}+\alpha_{4}+\alpha_{6} & \alpha_{2}+2 \alpha_{3}+2 \alpha_{4}+\alpha_{5}+\alpha_{6} \\
\alpha_{1}+2 \alpha_{2}+3 \alpha_{3}+2 \alpha_{4}+\alpha_{5}+\alpha_{6} & \alpha_{1}+\alpha_{2}+\alpha_{3}+\alpha_{6} & \alpha_{2}+2 \alpha_{3}+\alpha_{4}+\alpha_{5}+\alpha_{6} \\
\alpha_{1}+2 \alpha_{2}+2 \alpha_{3}+2 \alpha_{4}+\alpha_{5}+\alpha_{6} & \alpha_{1}+\alpha_{2}+\alpha_{3}+\alpha_{4}+\alpha_{5} & \alpha_{2}+2 \alpha_{3}+\alpha_{4}+\alpha_{6} \\
\alpha_{1}+2 \alpha_{2}+2 \alpha_{3}+\alpha_{4}+\alpha_{5}+\alpha_{6} & \alpha_{1}+\alpha_{2}+\alpha_{3}+\alpha_{4} & \alpha_{2}+\alpha_{3}+\alpha_{4}+\alpha_{5}+\alpha_{6} \\
\alpha_{1}+2 \alpha_{2}+2 \alpha_{3}+\alpha_{4}+\alpha_{6} & \alpha_{3}+\alpha_{4}+\alpha_{5} & \alpha_{2}+\alpha_{3}+\alpha_{4}+\alpha_{6} \\
\alpha_{1}+\alpha_{2}+2 \alpha_{3}+2 \alpha_{4}+\alpha_{5}+\alpha_{6} & \alpha_{3}+\alpha_{4}+\alpha_{6} & \alpha_{2}+\alpha_{3}+\alpha_{4}+\alpha_{5} \\
\alpha_{1}+\alpha_{2}+2 \alpha_{3}+\alpha_{4}+\alpha_{5}+\alpha_{6} & \alpha_{3}+\alpha_{4} & \alpha_{2}+\alpha_{3}+\alpha_{4} \\
\alpha_{1}+\alpha_{2}+2 \alpha_{3}+\alpha_{4}+\alpha_{6} & \alpha_{4}+\alpha_{5} & \alpha_{1}+\alpha_{2}+\alpha_{3} \\
\alpha_{1}+\alpha_{2}+\alpha_{3}+\alpha_{4}+\alpha_{5}+\alpha_{6} & \alpha_{4} & \alpha_{1}+\alpha_{2} \\
\alpha_{1} & \alpha_{3}+\alpha_{4}+\alpha_{5}+\alpha_{6} &
\end{array}\right.
$$

Let $\alpha=\sum_{k=1}^{6} c_{k} \alpha_{k} \in \Delta_{\mathfrak{n}}^{+}$. Since $\Pi_{\mathfrak{n}}=\left\{\alpha_{1}, \alpha_{4}\right\}$, by applying relation (17) we obtain that $\kappa(\alpha)=c_{1} \bar{\alpha}_{1}+c_{4} \bar{\alpha}_{4}$, where the numbers $c_{1}, c_{4}$ are such that $0 \leq c_{1}, c_{4} \leq 2$. So, by using (26), we easily conclude that the positive t-roots are given by $\Delta(\mathfrak{n})_{\mathfrak{t}}^{+}=\left\{\bar{\alpha}_{1}, \bar{\alpha}_{4}, \bar{\alpha}_{1}+\bar{\alpha}_{4}, 2 \bar{\alpha}_{4}, \bar{\alpha}_{1}+2 \bar{\alpha}_{4}\right\}$, and thus according to Proposition 3.5 (1), we obtain the decomposition (18) where the sumbodules $\mathfrak{n}_{i}$ are defined by (19). The sets $\Delta^{\mathfrak{n}}\left(j_{1}, j_{2}\right)$ are given explicitly as follows:

$$
\begin{aligned}
\Delta^{\mathfrak{n}}(1,0)= & \left\{\alpha_{1}, \alpha_{1}+\alpha_{2}, \alpha_{1}+\alpha_{2}+\alpha_{3}, \alpha_{1}+\alpha_{2}+\alpha_{3}+\alpha_{6}\right\} \\
\Delta^{\mathfrak{n}}(0,1)= & \left\{\alpha_{4}, \alpha_{3}+\alpha_{4}, \alpha_{4}+\alpha_{5}, \alpha_{2}+\alpha_{3}+\alpha_{4}, \alpha_{2}+\alpha_{3}+\alpha_{4}+\alpha_{5}, \alpha_{3}+\alpha_{4}+\alpha_{5}, \alpha_{3}+\alpha_{4}+\alpha_{5}+\alpha_{6}, \alpha_{3}+\alpha_{4}+\alpha_{6}\right. \\
& \left.\alpha_{2}+\alpha_{3}+\alpha_{4}+\alpha_{6}, \alpha_{2}+2 \alpha_{3}+\alpha_{4}+\alpha_{6}, \alpha_{2}+\alpha_{3}+\alpha_{4}+\alpha_{5}+\alpha_{6}, \alpha_{2}+2 \alpha_{3}+\alpha_{4}+\alpha_{5}+\alpha_{6}\right\} \\
\Delta^{\mathfrak{n}}(1,1)= & \left\{\alpha_{1}+\alpha_{2}+\alpha_{3}+\alpha_{4}, \alpha_{1}+\alpha_{2}+\alpha_{3}+\alpha_{4}+\alpha_{5}, \alpha_{1}+\alpha_{2}+\alpha_{3}+\alpha_{4}+\alpha_{6}, \alpha_{1}+\alpha_{2}+2 \alpha_{3}+\alpha_{4}+\alpha_{6},\right. \\
& \alpha_{1}+\alpha_{2}+\alpha_{3}+\alpha_{4}+\alpha_{5}+\alpha_{6}, \alpha_{1}+\alpha_{2}+2 \alpha_{3}+\alpha_{4}+\alpha_{5}+\alpha_{6}, \alpha_{1}+2 \alpha_{2}+2 \alpha_{3}+\alpha_{4}+\alpha_{5}+\alpha_{6}, \\
& \left.\alpha_{1}+2 \alpha_{2}+2 \alpha_{3}+\alpha_{4}+\alpha_{6}\right\} \\
\Delta^{\mathfrak{n}}(0,2)= & \left\{\alpha_{2}+2 \alpha_{3}+2 \alpha_{4}+\alpha_{5}+\alpha_{6}\right\} \\
\Delta^{\mathfrak{n}}(1,2)= & \left\{\alpha_{1}+\alpha_{2}+\alpha_{3}+2 \alpha_{4}+\alpha_{6}, \alpha_{1}+2 \alpha_{2}+3 \alpha_{3}+2 \alpha_{4}+\alpha_{5}+\alpha_{6}, \alpha_{1}+2 \alpha_{2}+2 \alpha_{3}+2 \alpha_{4}+\alpha_{5}+\alpha_{6},\right. \\
& \left.\alpha_{1}+2 \alpha_{2}+3 \alpha_{3}+2 \alpha_{4}+\alpha_{5}+2 \alpha_{6}\right\}
\end{aligned}
$$

By applying Proposition 3.5 (2) we easily conclude that

$$
\left.\begin{array}{rl}
\operatorname{dim}_{\mathbb{R}} \mathfrak{n}_{1}=2 \cdot\left|\left\{\alpha \in \Delta_{\mathfrak{n}}^{+}: \kappa(\alpha)=\bar{\alpha}_{1}\right\}\right|=2 \cdot\left|\Delta^{\mathfrak{n}}(1,0)\right|=2 \cdot 4=8, \\
\operatorname{dim}_{\mathbb{R}} \mathfrak{n}_{2}=2 \cdot\left|\left\{\alpha \in \Delta_{\mathfrak{n}}^{+}: \kappa(\alpha)=\bar{\alpha}_{4}\right\}\right|=2 \cdot\left|\Delta^{\mathfrak{n}}(0,1)\right|=2 \cdot 12=24, \\
\operatorname{dim}_{\mathbb{R}} \mathfrak{n}_{3}=2 \cdot\left|\left\{\alpha \in \Delta_{\mathfrak{n}}^{+}: \kappa(\alpha)=\bar{\alpha}_{1}+\bar{\alpha}_{4}\right\}\right|=2 \cdot\left|\Delta^{\mathfrak{n}}(1,1)\right|=2 \cdot 8=16, \\
\operatorname{dim}_{\mathbb{R}} \mathfrak{n}_{4}=2 \cdot\left|\left\{\alpha \in \Delta_{\mathfrak{n}}^{+}: \kappa(\alpha)=2 \bar{\alpha}_{4}\right\}\right|=2 \cdot\left|\Delta^{\mathfrak{n}}(0,2)\right|=2 \cdot 1=2, \\
\operatorname{dim}_{\mathbb{R}} \mathfrak{n}_{5}=2 \cdot\left|\left\{\alpha \in \Delta_{\mathfrak{n}}^{+}: \kappa(\alpha)=\bar{\alpha}_{1}+2 \bar{\alpha}_{4}\right\}\right|=2 \cdot\left|\Delta^{\mathfrak{n}}(1,2)\right|=2 \cdot 4=8 .
\end{array}\right\}
$$

Case of $\mathrm{E}_{6}$ : Type B. The flag manifold $\mathrm{E}_{6} / \mathrm{SU}(4) \times \mathrm{SU}(2) \times \mathrm{U}(1)^{2}$ is also defined by two pairs $\left(\Pi, \Pi_{0}\right)$ of Type B, given by $\Pi \backslash \Pi_{0}=\left\{\alpha_{4}, \alpha_{6}\right\}$ and $\Pi \backslash \Pi_{0}=\left\{\alpha_{2}, \alpha_{6}\right\}$. They correspond to the painted Dynkin diagrams
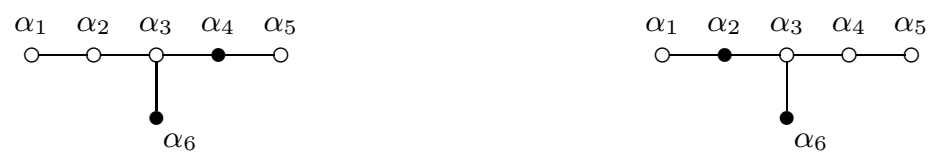

Note that there is also an outer automorphism of $\mathrm{E}_{6}$ which makes these painted Dynkin diagrams equivalent ( $\mathrm{BFR}]$ ), and thus we can work with the first pair $\left(\Pi, \Pi_{0}\right)$ only. By similar method we obtain that the positive $\mathfrak{t}$-roots are $\Delta(\mathfrak{m})_{\mathfrak{t}}^{+}=\left\{\bar{\alpha}_{6}, \bar{\alpha}_{4}, \bar{\alpha}_{6}+\bar{\alpha}_{4}, \bar{\alpha}_{6}+2 \bar{\alpha}_{4}, 2 \bar{\alpha}_{6}+2 \bar{\alpha}_{4}\right\}$ and thus according to Proposition 
3.5 (1), we obtain the decomposition (22) where the dimensions of the submodules $\mathfrak{m}_{i}$ are given as follows:

$$
\left.\begin{array}{l}
\operatorname{dim}_{\mathbb{R}} \mathfrak{m}_{1}=2 \cdot\left|\left\{\alpha \in \Delta_{\mathfrak{m}}^{+}: \kappa(\alpha)=\bar{\alpha}_{6}\right\}\right|=2 \cdot\left|\Delta^{\mathfrak{m}}(1,0)\right|=2 \cdot 4=8, \\
\operatorname{dim}_{\mathbb{R}} \mathfrak{m}_{2}=2 \cdot\left|\left\{\alpha \in \Delta_{\mathfrak{m}}^{+}: \kappa(\alpha)=\bar{\alpha}_{4}\right\}\right|=2 \cdot\left|\Delta^{\mathfrak{m}}(0,1)\right|=2 \cdot 8=16, \\
\operatorname{dim}_{\mathbb{R}} \mathfrak{m}_{3}=2 \cdot\left|\left\{\alpha \in \Delta_{\mathfrak{m}}^{+}: \kappa(\alpha)=\bar{\alpha}_{6}+\bar{\alpha}_{4}\right\}\right|=2 \cdot\left|\Delta^{\mathfrak{m}}(1,1)\right|=2 \cdot 12=24, \\
\operatorname{dim}_{\mathbb{R}} \mathfrak{m}_{4}=2 \cdot\left|\left\{\alpha \in \Delta_{\mathfrak{m}}^{+}: \kappa(\alpha)=\bar{\alpha}_{6}+2 \bar{\alpha}_{4}\right\}\right|=2 \cdot\left|\Delta^{\mathfrak{m}}(1,2)\right|=2 \cdot 4=8, \\
\operatorname{dim}_{\mathbb{R}} \mathfrak{m}_{5}=2 \cdot\left|\left\{\alpha \in \Delta_{\mathfrak{m}}^{+}: \kappa(\alpha)=2 \bar{\alpha}_{6}+2 \bar{\alpha}_{4}\right\}\right|=2 \cdot\left|\Delta^{\mathfrak{m}}(2,2)\right|=2 \cdot 1=2 .
\end{array}\right\}
$$

Case of $\mathrm{E}_{7}:$ Type A. Recall that the highest root $\tilde{\alpha}$ of $\mathrm{E}_{7}$ is given by $\tilde{\alpha}=\alpha_{1}+2 \alpha_{2}+3 \alpha_{3}+4 \alpha_{4}+$ $3 \alpha_{5}+2 \alpha_{6}+2 \alpha_{7}$. Consider the pair $\left(\Pi, \Pi_{0}\right)$ with $\Pi \backslash \Pi_{0}=\left\{\alpha_{1}, \alpha_{7}\right\}$. This choise corresponds to the painted Dynkin diagram

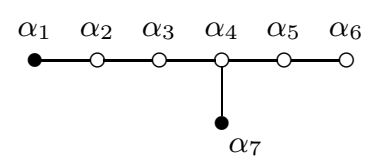

which determines the flag manifold $\mathrm{E}_{7} / \mathrm{SU}(6) \times \mathrm{U}(1)^{2}$. Let $\mathfrak{n}$ be a $B$-ortogonal complement of $\mathfrak{e}_{7}$. By using the expressions of positive roots of $\mathrm{E}_{7}$ in terms of the simple roots $\Pi=\left\{\alpha_{1}, \alpha_{2}, \alpha_{3}, \alpha_{4}, \alpha_{5}, \alpha_{6}, \alpha_{7}\right\}$ (see FrdV] or [Chr1] ) and by applying (17), we easily conclude that the positive $\mathfrak{t}$-roots are given by $\Delta(\mathfrak{n})_{\mathfrak{t}}^{+}=$ $\left\{\bar{\alpha}_{1}, \bar{\alpha}_{7}, \bar{\alpha}_{1}+\bar{\alpha}_{7}, 2 \bar{\alpha}_{7}, \bar{\alpha}_{1}+2 \bar{\alpha}_{7}\right\}$. Thus according to Proposition 3.5 (1) we obtain the decomposition (18), and the dimensions of the submodules $\mathfrak{n}_{i}$ are given as follows:

$$
\left.\begin{array}{rl}
\operatorname{dim}_{\mathbb{R}} \mathfrak{n}_{1}=2 \cdot\left|\left\{\alpha \in \Delta_{\mathfrak{n}}^{+}: \kappa(\alpha)=\bar{\alpha}_{1}\right\}\right|=2 \cdot\left|\Delta^{\mathfrak{n}}(1,0)\right|=2 \cdot 6, \\
\operatorname{dim}_{\mathbb{R}} \mathfrak{n}_{2}=2 \cdot\left|\left\{\alpha \in \Delta_{\mathfrak{n}}^{+}: \kappa(\alpha)=\bar{\alpha}_{7}\right\}\right|=2 \cdot\left|\Delta^{\mathfrak{n}}(0,1)\right|=2 \cdot 20, \\
\operatorname{dim}_{\mathbb{R}} \mathfrak{n}_{3}=2 \cdot\left|\left\{\alpha \in \Delta_{\mathfrak{n}}^{+}: \kappa(\alpha)=\bar{\alpha}_{1}+\bar{\alpha}_{7}\right\}\right|=2 \cdot\left|\Delta^{\mathfrak{n}}(1,1)\right|=2 \cdot 15, \\
\operatorname{dim}_{\mathbb{R}} \mathfrak{n}_{4}=2 \cdot\left|\left\{\alpha \in \Delta_{\mathfrak{n}}^{+}: \kappa(\alpha)=2 \bar{\alpha}_{7}\right\}\right|=2 \cdot\left|\Delta^{\mathfrak{n}}(0,2)\right|=2 \cdot 1, \\
\operatorname{dim}_{\mathbb{R}} \mathfrak{n}_{5}=2 \cdot\left|\left\{\alpha \in \Delta_{\mathfrak{n}}^{+}: \kappa(\alpha)=\bar{\alpha}_{1}+2 \bar{\alpha}_{7}\right\}\right|=2 \cdot\left|\Delta^{\mathfrak{n}}(1,2)\right|=2 \cdot 6 .
\end{array}\right\}
$$

Case of $\mathrm{E}_{7}$ : Type B. The flag manifold $\mathrm{E}_{7} / \mathrm{SU}(6) \times \mathrm{U}(1)^{2}$ is also defined by a pair $\left(\Pi, \Pi_{0}\right)$ of Type B, explicitely given by $\Pi \backslash \Pi_{0}=\left\{\alpha_{6}, \alpha_{7}\right\}$. It corrresponds to the painted Dynkin diagram

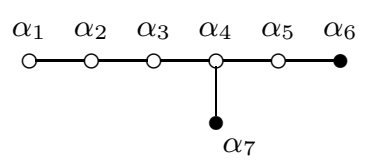

In this case the positive $\mathfrak{t}$-roots are given by $\Delta(\mathfrak{m})_{\mathfrak{t}}^{+}=\left\{\bar{\alpha}_{6}, \bar{\alpha}_{7}, \bar{\alpha}_{6}+\bar{\alpha}_{7}, \bar{\alpha}_{6}+2 \bar{\alpha}_{7}, 2 \bar{\alpha}_{6}+2 \bar{\alpha}_{7}\right\}$ and according to Proposition 3.5 (1), the $B$-orthogonal complement $\mathfrak{m}$ decomposes as (22), where the submodules $\mathfrak{m}_{i}$ have dimensions

$$
\left.\begin{array}{rl}
\operatorname{dim}_{\mathbb{R}} \mathfrak{m}_{1}=2 \cdot\left|\left\{\alpha \in \Delta_{\mathfrak{m}}^{+}: \kappa(\alpha)=\bar{\alpha}_{6}\right\}\right|=2 \cdot\left|\Delta^{\mathfrak{m}}(1,0)\right|=2 \cdot 6, \\
\operatorname{dim}_{\mathbb{R}} \mathfrak{m}_{2}=2 \cdot\left|\left\{\alpha \in \Delta_{\mathfrak{m}}^{+}: \kappa(\alpha)=\bar{\alpha}_{7}\right\}\right|=2 \cdot\left|\Delta^{\mathfrak{m}}(0,1)\right|=2 \cdot 15, \\
\operatorname{dim}_{\mathbb{R}} \mathfrak{m}_{3}=2 \cdot\left|\left\{\alpha \in \Delta_{\mathfrak{m}}^{+}: \kappa(\alpha)=\bar{\alpha}_{6}+\bar{\alpha}_{7}\right\}\right|=2 \cdot\left|\Delta^{\mathfrak{m}}(1,1)\right|=2 \cdot 20, \\
\operatorname{dim}_{\mathbb{R}} \mathfrak{m}_{4}=2 \cdot\left|\left\{\alpha \in \Delta_{\mathfrak{m}}^{+}: \kappa(\alpha)=\bar{\alpha}_{6}+2 \bar{\alpha}_{7}\right\}\right|=2 \cdot\left|\Delta^{\mathfrak{m}}(1,2)\right|=2 \cdot 6, \\
\operatorname{dim}_{\mathbb{R}} \mathfrak{m}_{5}=2 \cdot\left|\left\{\alpha \in \Delta_{\mathfrak{m}}^{+}: \kappa(\alpha)=2 \bar{\alpha}_{6}+2 \bar{\alpha}_{7}\right\}\right|=2 \cdot\left|\Delta^{\mathfrak{m}}(2,2)\right|=2 \cdot 1 .
\end{array}\right\}
$$

Step 2. By using [ACh3, Prop. 5] and Step 1 of the proof we have completed the study of all possible pairs $\left(\Pi, \Pi_{0}\right)$ of Type A. On the other hand, and due to the form of the highest root of the classical simple Lie groups, we have also studied all possible classical flag manifolds of Types A and B (the symplectic Lie group $\operatorname{Sp}(\ell)$ was treated in Example 3.6). Thus we now focus on pairs $\left(\Pi, \Pi_{0}\right)$ of Type B corresponding to exceptional Lie groups, which define flag manifolds with more than five isotropy summands. Hence these are not listed in Table 1.

Case of $\mathrm{E}_{6}$. For this Lie group there exists one more pair $\left(\Pi, \Pi_{0}\right)$ of Type B given by $\Pi \backslash \Pi_{0}=\left\{\alpha_{2}, \alpha_{4}\right\}$, which determines the flag manifold $\mathrm{E}_{6} / \mathrm{SU}(3) \times \mathrm{SU}(2) \times \mathrm{SU}(2) \times \mathrm{U}(1)^{2}$. The isotropy representation of this space decompsoses into six isotropy summands, since we find six positive $\mathfrak{t}$-roots given by $\left\{\bar{\alpha}_{2}, \bar{\alpha}_{4}, \bar{\alpha}_{2}+\right.$ $\left.\bar{\alpha}_{4}, \bar{\alpha}_{2}+2 \bar{\alpha}_{4}, 2 \bar{\alpha}_{2}+\bar{\alpha}_{4}, 2 \bar{\alpha}_{2}+2 \bar{\alpha}_{4}\right\}$. 
Case of $E_{7}$. In this case there are two more pairs $\left(\Pi, \Pi_{0}\right)$ of Type B, namely the pairs $\Pi \backslash \Pi_{0}=\left\{\alpha_{2}, \alpha_{7}\right\}$ and $\Pi \backslash \Pi_{0}=\left\{\alpha_{2}, \alpha_{6}\right\}$ which determine the flag manifolds $\mathrm{E}_{7} / \mathrm{SU}(5) \times \mathrm{SU}(2) \times \mathrm{U}(1)^{2}$ and $\mathrm{E}_{7} / \mathrm{SO}(8) \times \mathrm{SU}(2) \times$ $\mathrm{U}(1)^{2}$ respectively. Both of these flag manifolds have six isotropy summands, since the associated positive t-roots are given by $\left\{\bar{\alpha}_{2}, \bar{\alpha}_{7}, \bar{\alpha}_{2}+\bar{\alpha}_{7}, 2 \bar{\alpha}_{2}+\bar{\alpha}_{7}, \bar{\alpha}_{2}+2 \bar{\alpha}_{7}, 2 \bar{\alpha}_{2}+2 \bar{\alpha}_{7}\right\}$ and $\left\{\bar{\alpha}_{2}, \bar{\alpha}_{6}, \bar{\alpha}_{2}+\bar{\alpha}_{6}, 2 \bar{\alpha}_{2}, 2 \bar{\alpha}_{2}+\right.$ $\left.\bar{\alpha}_{6}, 2 \bar{\alpha}_{2}+2 \bar{\alpha}_{6}\right\}$ respectively.

Case of $\mathrm{E}_{8}$. The highest root $\tilde{\alpha}$ of $\mathrm{E}_{8}$ is given by $\tilde{\alpha}=2 \alpha_{1}+3 \alpha_{2}+4 \alpha_{3}+5 \alpha_{4}+6 \alpha_{5}+4 \alpha_{6}+2 \alpha_{7}+3 \alpha_{8}$. Thus for $\mathrm{E}_{8}$ there exists only a pair $\left(\Pi, \Pi_{0}\right)$ of Type $\mathrm{B}$, given by $\Pi \backslash \Pi_{0}=\left\{\alpha_{1}, \alpha_{7}\right\}$. It determines the flag manifold $\mathrm{E}_{8} / \mathrm{SO}(12) \times \mathrm{U}(1)^{2}$ which has six isotropy summands. Indeed, by expressing the positive roots in terms of simple roots (see [Chr1] or [FrdV]), and by applying (17) we obtain six positive t-roots, namely $\left\{\bar{\alpha}_{1}, \bar{\alpha}_{7}, \bar{\alpha}_{1}+\bar{\alpha}_{7}, 2 \bar{\alpha}_{1}+\bar{\alpha}_{7}, 2 \bar{\alpha}_{7}, 2 \bar{\alpha}_{1}+2 \bar{\alpha}_{7}\right\}$.

Case of $\mathrm{F}_{4}$. The highest root $\tilde{\alpha}$ of $\mathrm{F}_{4}$ is given by $\tilde{\alpha}=2 \alpha_{1}+3 \alpha_{2}+4 \alpha_{3}+2 \alpha_{4}$. Thus, the unique pair ( $\left.\Pi, \Pi_{0}\right)$ of Type B is given by $\Pi \backslash \Pi_{0}=\left\{\alpha_{1}, \alpha_{4}\right\}$. It determines the flag manifold $\mathrm{F}_{4} / \mathrm{SO}(5) \times \mathrm{U}(1)^{2}$ with six isotropy summands. Indeed, by using the expressions of positive roots in terms of simple roots (see $\mathrm{AlAr}$ ) and by applying (17) we obtain six positive $t$-roots, namely $\left\{\bar{\alpha}_{1}, \bar{\alpha}_{4}, \bar{\alpha}_{1}+\bar{\alpha}_{4}, 2 \bar{\alpha}_{1}+\bar{\alpha}_{4}, 2 \bar{\alpha}_{1}, 2 \bar{\alpha}_{1}+2 \bar{\alpha}_{4}\right\}$.

The following corollary in now immediate.

Corollary 4.3. The only generalized flag manifolds $M$ with $b_{2}(M)=2$ whose isotropy representation decomposes into five isotropy summands are those listed in Table 1.

Corollary 4.4. Let $M=G / K$ be a flag manifold of Type $A$ with isotropy representation $\mathfrak{n}=\mathfrak{n}_{1} \oplus \mathfrak{n}_{2} \oplus$ $\mathfrak{n}_{3} \oplus \mathfrak{n}_{4} \oplus \mathfrak{n}_{5}$. Then the $\operatorname{Ad}(K)$-modules $\mathfrak{n}_{i}$ satisfy the relations $\left[\mathfrak{n}_{1}, \mathfrak{n}_{2}\right]=\mathfrak{n}_{3},\left[\mathfrak{n}_{1}, \mathfrak{n}_{4}\right]=\mathfrak{n}_{5},\left[\mathfrak{n}_{2}, \mathfrak{n}_{2}\right] \subset \mathfrak{n}_{4} \oplus \mathfrak{k}$, $\left[\mathfrak{n}_{2}, \mathfrak{n}_{3}\right]=\mathfrak{n}_{1} \oplus \mathfrak{n}_{5},\left[\mathfrak{n}_{1}, \mathfrak{n}_{3}\right]=\mathfrak{n}_{2},\left[\mathfrak{n}_{2}, \mathfrak{n}_{4}\right]=\mathfrak{n}_{2},\left[\mathfrak{n}_{1}, \mathfrak{n}_{5}\right]=\mathfrak{n}_{4},\left[\mathfrak{n}_{4}, \mathfrak{n}_{5}\right]=\mathfrak{n}_{1},\left[\mathfrak{n}_{2}, \mathfrak{n}_{5}\right]=\mathfrak{n}_{3}$, and $\left[\mathfrak{n}_{3}, \mathfrak{n}_{5}\right]=\mathfrak{n}_{2}$.

Proof. It is immediate by considering the $T$-root systems of the flag manifolds of Type A in the proof of Proposition 4.2

4.3. The isometry between flag manifolds of Type A and Type B. By using the analysis given in the previous paragraph, we will prove that for any simple Lie group $G$ appearing in Table 1, there is an isometry which makes the corresponding flag manifolds $G / K$ with five isotropy summands of Type A and B, isometric as real manifolds. We will show that this isometry is obtained in a canonical way from the Weyl group $\mathcal{W}$ corresponding to (the root system of) $G$.

Theorem 4.5. For any Lie group $G$ appearing in Table 1, the correpsonding pairs $\left(\Pi, \Pi_{0}\right)$ of Type $A$ and $B$, define isometric flag manifolds (as real manifolds).

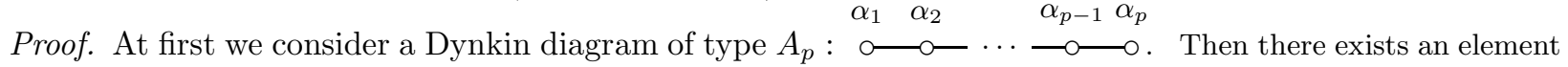
$w_{0}$ of the Weyl group $\mathcal{W}$ of $\mathrm{SU}(p+1)$ with $w_{0}\left(\alpha_{i}\right)=-\alpha_{p+1-i}$ for $i=1, \ldots, p$. In fact, $w_{0}$ is given by

$$
w_{0}=s_{\alpha_{k+1}} \cdot s_{\alpha_{k}+\alpha_{k+1}+\alpha_{k+2}} \cdots s_{\alpha_{2}+\cdots+\alpha_{k+1}+\cdots+\alpha_{p-1}} \cdot s_{\alpha_{1}+\cdots+\alpha_{k+1}+\cdots+\alpha_{p}} \text { for } p=2 k+1
$$

and

$$
w_{0}=s_{\alpha_{k}+\alpha_{k+1}} \cdot s_{\alpha_{k}+\alpha_{k+1}+\alpha_{k+2}} \cdots s_{\alpha_{2}+\cdots+\alpha_{k+1}+\cdots+\alpha_{p-1}} \cdot s_{\alpha_{1}+\cdots+\alpha_{k+1}+\cdots+\alpha_{p}} \text { for } p=2 k,
$$

where $s_{\beta}$ is the reflection defined by a $\operatorname{root} \beta$.

For a moment we write $\Pi_{0}(A)$ and $\Pi_{0}(B)$ for $\Pi_{0}$ of Type $\mathrm{A}$ and $\Pi_{0}$ of Type $\mathrm{B}$ and also write $\Delta_{0}(A)$ and $\Delta_{0}(B)$ for $\Delta_{0}$ of Type A and $\Delta_{0}$ of Type B respectively. Now for $B_{\ell}$ and $D_{\ell}$ we see that the pair $\left(\Pi, \Pi_{0}\right)$ is given by $\Pi_{\mathfrak{n}}=\Pi \backslash \Pi_{0}=\left\{\alpha_{1}, \alpha_{p+1}\right\}$ (Type A) and $\Pi \backslash \Pi_{0}=\Pi_{\mathfrak{m}}=\left\{\alpha_{p}, \alpha_{p+1}\right\}$ (Type B) and thus the painted Dynkin diagram of Type A and Type B contain a Dynkin subdiagram of type $A_{p}$, where $p=2, \ldots, \ell-1$ for $B_{\ell}$ and $p=2, \ldots, \ell-3$ for $D_{\ell}$. We regard $w_{0}$ as an element of the Weyl group of type $B_{\ell}$ and $D_{\ell}$. Then we have $w_{0}\left(\alpha_{p+1}\right)=\alpha_{p+1}+\left(\alpha_{1}+\cdots+\alpha_{p}\right)$ and $w_{0}\left(\alpha_{p+k}\right)=\alpha_{p+k}$ for $k=2, \ldots, \ell-p$, and it follows that $w_{0}\left(\Delta_{0}(A)\right)=\Delta_{0}(B)$ and

$$
\begin{aligned}
& w_{0}\left(\Delta^{\mathfrak{n}}(1,0)\right)=-\Delta^{\mathfrak{m}}(1,0), \quad w_{0}\left(\Delta^{\mathfrak{n}}(0,1)\right)=\Delta^{\mathfrak{m}}(1,1), \quad w_{0}\left(\Delta^{\mathfrak{n}}(1,1)\right)=\Delta^{\mathfrak{m}}(0,1), \\
& w_{0}\left(\Delta^{\mathfrak{n}}(0,2)\right)=\Delta^{\mathfrak{m}}(2,2), \quad w_{0}\left(\Delta^{\mathfrak{n}}(1,2)\right)=\Delta^{\mathfrak{m}}(1,2) .
\end{aligned}
$$

For $\mathrm{E}_{6}$ the pair $\left(\Pi, \Pi_{0}\right)$ is given by $\Pi_{\mathfrak{n}}=\Pi \backslash \Pi_{0}=\left\{\alpha_{1}, \alpha_{4}\right\}$ (Type A) and $\Pi \backslash \Pi_{0}=\Pi_{\mathfrak{m}}=\left\{\alpha_{4}, \alpha_{6}\right\}$ (Type B) and thus the painted Dynkin diagrams of Type A and Type B contain a Dynkin subdiagram of type $A_{4}$ : 
\begin{tabular}{cccc}
$\alpha_{1}$ & $\alpha_{2}$ & $\alpha_{3}$ & $\alpha_{6}$ \\
\hdashline & - & 0 & 0
\end{tabular} . Let $w_{0}$ be the element of the Weyl group $\mathcal{W}$ of SU(5) given by $w_{0}=s_{\alpha_{2}+\alpha_{3}} \cdot s_{\alpha_{1}+\alpha_{2}+\alpha_{3}+\alpha_{6}}$. We regard $w_{0}$ as an element of the Weyl group of type $\mathrm{E}_{6}$. Then we have that $w_{0}\left(\alpha_{1}\right)=-\alpha_{6}, w_{0}\left(\alpha_{2}\right)=-\alpha_{3}$, $w_{0}\left(\alpha_{3}\right)=-\alpha_{2}, w_{0}\left(\alpha_{6}\right)=-\alpha_{1}, w_{0}\left(\alpha_{4}\right)=\alpha_{1}+2 \alpha_{2}+2 \alpha_{3}+\alpha_{4}+\alpha_{6}$ and $w_{0}\left(\alpha_{5}\right)=\alpha_{5}$. Thus we get $w_{0}\left(\Delta_{0}(A)\right)=\Delta_{0}(B)$ and

$$
\begin{aligned}
& w_{0}\left(\Delta^{\mathfrak{n}}(1,0)\right)=-\Delta^{\mathfrak{m}}(1,0), \quad w_{0}\left(\Delta^{\mathfrak{n}}(0,1)\right)=\Delta^{\mathfrak{m}}(1,1), \quad w_{0}\left(\Delta^{\mathfrak{n}}(1,1)\right)=\Delta^{\mathfrak{m}}(0,1), \\
& w_{0}\left(\Delta^{\mathfrak{n}}(0,2)\right)=\Delta^{\mathfrak{m}}(2,2), \quad w_{0}\left(\Delta^{\mathfrak{n}}(1,2)\right)=\Delta^{\mathfrak{m}}(1,2) .
\end{aligned}
$$

For $\mathrm{E}_{7}$ we see that the pair $\left(\Pi, \Pi_{0}\right)$ is given by $\Pi_{\mathfrak{n}}=\Pi \backslash \Pi_{0}=\left\{\alpha_{1}, \alpha_{7}\right\}$ (Type A) and $\Pi \backslash \Pi_{0}=\Pi_{\mathfrak{m}}=\left\{\alpha_{6}, \alpha_{7}\right\}$ (Type B) and thus the painted Dynkin diagrams of Type A and Type B contain a Dynkin subdiagram of type $A_{6}$ :

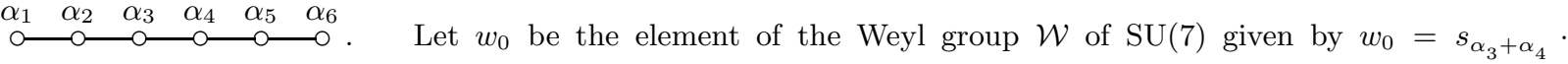
$s_{\alpha_{2}+\alpha_{3}+\alpha_{4}+\alpha_{5}} \cdot s_{\alpha_{1}+\alpha_{2}+\alpha_{3}+\alpha_{4}+\alpha_{5}+\alpha_{6}}$. We regard $w_{0}$ as an element of the Weyl group of type $E_{7}$. Then we have that $w_{0}\left(\alpha_{1}\right)=-\alpha_{6}, w_{0}\left(\alpha_{2}\right)=-\alpha_{5}, w_{0}\left(\alpha_{3}\right)=-\alpha_{4}, w_{0}\left(\alpha_{4}\right)=-\alpha_{3}, w_{0}\left(\alpha_{5}\right)=-\alpha_{2}, w_{0}\left(\alpha_{6}\right)=-\alpha_{1}$, $w_{0}\left(\alpha_{7}\right)=\alpha_{1}+2 \alpha_{2}+3 \alpha_{3}+3 \alpha_{4}+2 \alpha_{5}+\alpha_{6}+\alpha_{7}$. TIt follows that $w_{0}\left(\Delta_{0}(A)\right)=\Delta_{0}(B)$ and

$$
\begin{aligned}
& w_{0}\left(\Delta^{\mathfrak{n}}(1,0)\right)=-\Delta^{\mathfrak{m}}(1,0), \quad w_{0}\left(\Delta^{\mathfrak{n}}(0,1)\right)=\Delta^{\mathfrak{m}}(1,1), \quad w_{0}\left(\Delta^{\mathfrak{n}}(1,1)\right)=\Delta^{\mathfrak{m}}(0,1), \\
& w_{0}\left(\Delta^{\mathfrak{n}}(0,2)\right)=\Delta^{\mathfrak{m}}(2,2), \quad w_{0}\left(\Delta^{\mathfrak{n}}(1,2)\right)=\Delta^{\mathfrak{m}}(1,2) .
\end{aligned}
$$

Hence we get an isometry between the corresponding tangent spaces $\mathfrak{n}$ (Type A) and $\mathfrak{m}$ (Type B) and, therefore we obtain an isometry between flag manifolds of Type A and Type B.

\section{KÄHLER-EINSTEIN METRICS}

In computing the Ricci tensor for a generalized flag manifold $M=G / K$ by using Riemannian submersions we will use the well known fact that $M$ admits a finite number of $G$-invariant Kähler-Einstein metrics. Recall that if $M=G / K$ is determined by a pair $\left(\Pi, \Pi_{K}\right)$ with reductive decomposition $\mathfrak{g}=\mathfrak{k} \oplus \mathfrak{m}$, then $G$-invariant complex structures are in one-to-one correspondence with invariant orderings $\Delta_{\mathfrak{m}}^{+}$in $\Delta_{\mathfrak{m}}($ Ale1], [Bor, p. 625]). Put $Z_{\mathfrak{t}}=\left\{\Lambda \in \mathfrak{t} \mid \frac{2(\Lambda, \alpha)}{(\alpha, \alpha)} \in \mathbb{Z}\right.$ for each $\left.\alpha \in \Delta\right\}$. Then $Z_{\mathfrak{t}}$ is a lattice of $\mathfrak{t}$ generated by the fundamental weights $\left\{\Lambda_{i_{1}}, \cdots, \Lambda_{i_{r}}\right\}$. Set $Z_{\mathfrak{t}}^{+}=\left\{\lambda \in Z_{\mathfrak{t}} \mid(\lambda, \alpha)>0\right.$ for $\alpha \in \Pi \backslash \Pi$. Then we have $Z_{\mathfrak{t}}^{+}=\sum_{\alpha \in \Pi \backslash \Pi_{0}} \mathbb{Z}^{+} \Lambda_{\alpha}$ and define the element $\delta_{\mathfrak{m}}=\frac{1}{2} \sum_{\alpha \in \Delta_{\mathfrak{m}}^{+}} \alpha \in \sqrt{-1} \mathfrak{h}$. Put $k_{\alpha}=\frac{2\left(2 \delta_{\mathfrak{m}}, \alpha\right)}{(\alpha, \alpha)}$ for $\alpha \in \Pi \backslash \Pi$. Then $2 \delta_{\mathfrak{m}}=\sum_{\alpha \in \Pi \backslash \Pi_{0}} k_{\alpha} \Lambda_{\alpha}=k_{\alpha_{i_{1}}} \Lambda_{\alpha_{i_{1}}}+\cdots+k_{\alpha_{i_{r}}} \Lambda_{\alpha_{i_{r}}}$ and each $k_{\alpha_{i_{s}}}$ is a positive integer. We have the following:

Proposition 5.1. The $G$-invariant metric $g_{2 \delta_{\mathfrak{m}}}$ on $G / K$ corresponding to $2 \delta_{\mathfrak{m}}$ is a Kähler Einstein metric which is given by

$$
g_{2 \delta_{\mathfrak{m}}}=\left.\sum_{j_{1}, \cdots, j_{r}}\left(\sum_{\ell=1}^{r} k_{\alpha_{i_{\ell}}} j_{\ell} \frac{\left(\alpha_{j_{\ell}}, \alpha_{j_{\ell}}\right)}{2}\right) B\right|_{\mathfrak{m}\left(j_{1}, \cdots, j_{r}\right)}
$$

Example 5.2. Case of $B_{\ell}=\mathrm{SO}(2 \ell+1)$ : Type A. Let $\Pi \backslash \Pi_{0}=\Pi_{\mathfrak{n}}=\left\{\alpha_{1}, \alpha_{p+1}: 2 \leq p \leq \ell-1\right\}$. For the flag manifold $M=\mathrm{SO}(2 \ell+1) /(\mathrm{U}(1) \times \mathrm{U}(p) \times \mathrm{SO}(2(\ell-p-1)+1))$ with $2 \leq p \leq \ell-1$ and $\ell \geq 3$, we see that $2 \delta_{\mathfrak{n}}=(p+1) \Lambda_{\alpha_{1}}+(2 \ell-p-2) \Lambda_{\alpha_{p+1}}$. Thus the Kähler Einstein metric $g_{2 \delta_{\mathfrak{n}}}$ on $G / K$ is given by

$$
\begin{array}{r}
g_{2 \delta_{\mathfrak{n}}}=\left.(p+1) B\right|_{\mathfrak{n}(1,0)}+\left.(2 \ell-p-2) B\right|_{\mathfrak{n}(0,1)}+\left.(2 \ell-1) B\right|_{\mathfrak{n}(1,1)} \\
+\left.2(2 \ell-p-2) B\right|_{\mathfrak{n}(0,2)}+\left.(4 \ell-p-3) B\right|_{\mathfrak{n}(1,2)} .
\end{array}
$$

Example 5.3. Case of $D_{\ell}=\mathrm{SO}(2 \ell)$ : Type A. Let $\Pi \backslash \Pi_{0}=\Pi_{\mathfrak{n}}=\left\{\alpha_{1}, \alpha_{p+1}: 2 \leq p \leq \ell-3\right\}$. For the flag manifold $M=\mathrm{SO}(2 \ell) /(\mathrm{U}(1) \times \mathrm{U}(p) \times \mathrm{SO}(2(\ell-p-1)))$ with $2 \leq p \leq \ell-3$ and $\ell \geq 5$, we see that $2 \delta_{\mathfrak{n}}=(p+1) \Lambda_{\alpha_{1}}+(2 \ell-p-3) \Lambda_{\alpha_{p+1}}$. Thus the Kähler Einstein metric $g_{2 \delta_{\mathfrak{n}}}$ on $G / K$ is given by

$$
\begin{array}{r}
g_{2 \delta_{\mathfrak{n}}}=\left.(p+1) B\right|_{\mathfrak{n}(1,0)}+\left.(2 \ell-p-3) B\right|_{\mathfrak{n}(0,1)}+\left.(2 \ell-2) B\right|_{\mathfrak{n}(1,1)} \\
+\left.2(2 \ell-p-3) B\right|_{\mathfrak{n}(0,2)}+\left.(4 \ell-p-5) B\right|_{\mathfrak{n}(1,2)} .
\end{array}
$$


Example 5.4. Case of $\mathrm{E}_{6}$ : Type A. Let $\Pi \backslash \Pi_{0}=\Pi_{\mathfrak{n}}=\left\{\alpha_{1}, \alpha_{4}\right\}$. For the flag manifold $M=\mathrm{E}_{6} /(\mathrm{U}(4) \times$ $\mathrm{U}(2))$ we see that $2 \delta_{\mathfrak{n}}=5 \Lambda_{\alpha_{1}}+7 \Lambda_{\alpha_{4}}$. Thus the Kähler Einstein metric $g_{2 \delta_{\mathfrak{n}}}$ on $G / K$ is given by

$$
g_{2 \delta_{\mathfrak{n}}}=\left.5 B\right|_{\mathfrak{n}(1,0)}+\left.7 B\right|_{\mathfrak{n}(0,1)}+\left.12 B\right|_{\mathfrak{n}(1,1)}+\left.14 B\right|_{\mathfrak{n}(0,2)}+\left.19 B\right|_{\mathfrak{n}(1,2)} .
$$

Example 5.5. Case of $\mathrm{E}_{6}$ : Type B. Let $\Pi \backslash \Pi_{0}=\Pi_{\mathfrak{m}}=\left\{\alpha_{6}, \alpha_{4}\right\}$. For the flag manifold $M=\mathrm{E}_{6} /(\mathrm{U}(4) \times$ $\mathrm{U}(2))$ we see that $2 \delta_{\mathfrak{m}}=5 \Lambda_{\alpha_{6}}+6 \Lambda_{\alpha_{4}}$. Thus the Kähler Einstein metric $g_{2 \delta_{\mathfrak{m}}}$ on $G / K$ is given by

$$
g_{2 \delta_{\mathfrak{m}}}=\left.5 B\right|_{\mathfrak{m}(1,0)}+\left.6 B\right|_{\mathfrak{m}(0,1)}+\left.11 B\right|_{\mathfrak{m}(1,1)}+\left.17 B\right|_{\mathfrak{m}(1,2)}+\left.22 B\right|_{\mathfrak{m}(2,2)} .
$$

Example 5.6. Case of $\mathrm{E}_{7}$ : Type A. Let $\Pi \backslash \Pi_{0}=\Pi_{\mathfrak{n}}=\left\{\alpha_{1}, \alpha_{7}\right\}$. For the flag manifold $M=\mathrm{E}_{7} /(\mathrm{U}(1) \times$ $\mathrm{U}(6))$ we see that $2 \delta_{\mathfrak{n}}=7 \Lambda_{\alpha_{1}}+11 \Lambda_{\alpha_{7}}$. Thus the Kähler Einstein metric $g_{2 \delta_{\mathfrak{n}}}$ on $G / K$ is given by

$$
g_{2 \delta_{\mathfrak{n}}}=\left.7 B\right|_{\mathfrak{n}(1,0)}+\left.11 B\right|_{\mathfrak{n}(0,1)}+\left.18 B\right|_{\mathfrak{n}(1,1)}+\left.22 B\right|_{\mathfrak{n}(0,2)}+\left.29 B\right|_{\mathfrak{n}(1,2)} .
$$

Example 5.7. Case of $\mathrm{E}_{7}$ : Type B. Let $\Pi \backslash \Pi_{0}=\Pi_{\mathfrak{n}}=\left\{\alpha_{6}, \alpha_{7}\right\}$. For the flag manifold $M=\mathrm{E}_{7} /(\mathrm{U}(1) \times$ $\mathrm{U}(6))$ we see that $2 \delta_{\mathfrak{m}}=7 \Lambda_{\alpha_{6}}+10 \Lambda_{\alpha_{7}}$. Thus the Kähler Einstein metric $g_{2 \delta_{\mathfrak{m}}}$ on $G / K$ is given by

$$
g_{2 \delta_{\mathfrak{m}}}=\left.7 B\right|_{\mathfrak{m}(1,0)}+\left.10 B\right|_{\mathfrak{m}(0,1)}+\left.17 B\right|_{\mathfrak{m}(1,1)}+\left.27 B\right|_{\mathfrak{m}(1,2)}+\left.34 B\right|_{\mathfrak{m}(2,2)} .
$$

\section{The Ricci tensor on Flag MANifolds With Five isotropy SUmmandS}

We now proceed to the calculation of the Ricci tensor $r$ corresponding to a $G$-invariant metric (3) on $G / K$ of Type A. In order to apply Lemma 2.1 we first need to find the non zero structure constants $\left[\begin{array}{c}k \\ i j\end{array}\right]$ of $G / K$. Due to the bracket relations in Corollary 4.4 we obtain that the non zero structure constant are

$$
\left[\begin{array}{c}
3 \\
12
\end{array}\right],\left[\begin{array}{c}
4 \\
22
\end{array}\right],\left[\begin{array}{c}
5 \\
23
\end{array}\right],\left[\begin{array}{c}
5 \\
14
\end{array}\right] \text {. }
$$

We write $G$-invariant metrics $g$ on $G / K$ as

$$
g=\left.x_{1} B\right|_{\mathfrak{n}_{1}}+\left.x_{2} B\right|_{\mathfrak{n}_{2}}+\left.x_{3} B\right|_{\mathfrak{n}_{3}}+\left.x_{4} B\right|_{\mathfrak{n}_{4}}+\left.x_{5} B\right|_{\mathfrak{n}_{5}}
$$

where $x_{j}(j=1, \ldots, 5)$ are positive numbers.

Put $d_{i}=\operatorname{dim} \mathfrak{n}_{i}$ for $i=1, \ldots, 5$. From Lemma 2.1 we obtain the following proposition.

Proposition 6.1. The components $r_{i}(i=1, \ldots, 5)$ of the Ricci tensor for a $G$-invariant Riemannian metric (31) on $G / K$ are given as follows:

$$
\begin{aligned}
& r_{1}=\frac{1}{2 x_{1}}+\frac{1}{2 d_{1}}\left[\begin{array}{c}
3 \\
12
\end{array}\right]\left(\frac{x_{1}}{x_{2} x_{3}}-\frac{x_{2}}{x_{1} x_{3}}-\frac{x_{3}}{x_{1} x_{2}}\right)+\frac{1}{2 d_{1}}\left[\begin{array}{c}
5 \\
14
\end{array}\right]\left(\frac{x_{1}}{x_{4} x_{5}}-\frac{x_{5}}{x_{1} x_{4}}-\frac{x_{4}}{x_{1} x_{5}}\right), \\
& r_{2}=\frac{1}{2 x_{2}}+\frac{1}{2 d_{2}}\left[\begin{array}{c}
3 \\
12
\end{array}\right]\left(\frac{x_{2}}{x_{1} x_{3}}-\frac{x_{1}}{x_{2} x_{3}}-\frac{x_{3}}{x_{1} x_{2}}\right)-\frac{1}{2 d_{2}}\left[\begin{array}{c}
4 \\
22
\end{array}\right] \frac{x_{4}}{x_{2}{ }^{2}}+\frac{1}{2 d_{2}}\left[\begin{array}{c}
5 \\
23
\end{array}\right]\left(\frac{x_{2}}{x_{3} x_{5}}-\frac{x_{5}}{x_{2} x_{3}}-\frac{x_{3}}{x_{2} x_{5}}\right), \\
& r_{3}=\frac{1}{2 x_{3}}+\frac{1}{2 d_{3}}\left[\begin{array}{c}
3 \\
12
\end{array}\right]\left(\frac{x_{3}}{x_{1} x_{2}}-\frac{x_{2}}{x_{1} x_{3}}-\frac{x_{1}}{x_{2} x_{3}}\right)+\frac{1}{2 d_{3}}\left[\begin{array}{c}
5 \\
23
\end{array}\right]\left(\frac{x_{3}}{x_{2} x_{5}}-\frac{x_{5}}{x_{2} x_{3}}-\frac{x_{2}}{x_{3} x_{5}}\right), \\
& r_{4}=\frac{1}{2 x_{4}}+\frac{1}{2 d_{4}}\left[\begin{array}{c}
5 \\
14
\end{array}\right]\left(\frac{x_{4}}{x_{1} x_{5}}-\frac{x_{5}}{x_{1} x_{4}}-\frac{x_{1}}{x_{4} x_{5}}\right)+\frac{1}{4 d_{4}}\left[\begin{array}{c}
4 \\
22
\end{array}\right]\left(-\frac{2}{x_{4}}+\frac{x_{4}}{x_{2}{ }^{2}}\right), \\
& r_{5}=\frac{1}{2 x_{5}}+\frac{1}{2 d_{5}}\left[\begin{array}{c}
5 \\
23
\end{array}\right]\left(\frac{x_{5}}{x_{2} x_{3}}-\frac{x_{2}}{x_{3} x_{5}}-\frac{x_{3}}{x_{2} x_{5}}\right)+\frac{1}{2 d_{5}}\left[\begin{array}{c}
5 \\
14
\end{array}\right]\left(\frac{x_{5}}{x_{1} x_{4}}-\frac{x_{1}}{x_{4} x_{5}}-\frac{x_{4}}{x_{1} x_{5}}\right) .
\end{aligned}
$$

Let $\mathfrak{k}$ be the subalgebra of $\mathfrak{g}$ corresponding to the Lie subgroup $K$. We consider a subspace $\mathfrak{l}=\mathfrak{k} \oplus \mathfrak{n}_{1}$ of $\mathfrak{g}$. Then $\mathfrak{l}$ is a subalgebra of $\mathfrak{g}$ and we have a natural fibration $\pi: G / K \rightarrow G / L$ with fiber $L / K$. We decompose $\mathfrak{p}=\mathfrak{p}_{1} \oplus \mathfrak{p}_{2}$ and $\mathfrak{q}=\mathfrak{q}_{1}$, where $\mathfrak{p}_{1}=\mathfrak{n}_{2} \oplus \mathfrak{n}_{3}=\mathfrak{m}_{1,1} \oplus \mathfrak{m}_{1,2}, \mathfrak{p}_{2}=\mathfrak{n}_{4} \oplus \mathfrak{n}_{5}=\mathfrak{m}_{2,1} \oplus \mathfrak{m}_{2,2}$ and $\mathfrak{q}_{1}=\mathfrak{n}_{1}$. We consider a $G$-invariant metric on $G / K$ defined by a Riemannian submersion $\pi:(G / K, g) \rightarrow(G / L, \check{g})$ given by

$$
g=\left.y_{1} B\right|_{\mathfrak{p}_{1}}+\left.y_{2} B\right|_{\mathfrak{p}_{2}}+\left.z_{1} B\right|_{\mathfrak{q}_{1}}
$$


and the metric $\check{g}$ on $G / L$

$$
\check{g}=\left.y_{1} B\right|_{\mathfrak{p}_{1}}+\left.y_{2} B\right|_{\mathfrak{p}_{2}}
$$

for positive real numbers $y_{1}, y_{2}, z_{1}$. Note that, when we write the metric (33) as in the form (31), we have

$$
g=\left.y_{1} B\right|_{\mathfrak{n}_{2}}+\left.y_{1} B\right|_{\mathfrak{n}_{3}}+\left.y_{2} B\right|_{\mathfrak{n}_{4}}+\left.y_{2} B\right|_{\mathfrak{n}_{5}}+\left.z_{1} B\right|_{\mathfrak{n}_{1}} .
$$

From (32) we obtain the components $r_{i}$ of the Ricci tensor for the metric (34) on $G / K$ as follows:

$$
\begin{gathered}
r_{3}=\frac{1}{2 y_{1}}-\frac{1}{2 d_{3}}\left[\begin{array}{c}
3 \\
12
\end{array}\right] \frac{z_{1}}{y_{1}{ }^{2}}-\frac{1}{2 d_{3}}\left[\begin{array}{c}
5 \\
23
\end{array}\right] \frac{y_{2}}{y_{1}^{2}}, \\
r_{4}=\frac{1}{2 y_{2}}-\frac{1}{2 d_{4}}\left[\begin{array}{c}
5 \\
14
\end{array}\right] \frac{z_{1}}{y_{2}{ }^{2}}+\frac{1}{4 d_{4}}\left[\begin{array}{c}
4 \\
22
\end{array}\right]\left(\frac{y_{2}}{y_{1}^{2}}-\frac{2}{y_{2}}\right) .
\end{gathered}
$$

We put $\tilde{d}_{1}=\operatorname{dim} \mathfrak{p}_{1}$ and $\tilde{d}_{2}=\operatorname{dim} \mathfrak{p}_{2}$. Then $\tilde{d}_{1}=d_{2}+d_{3}$ and $\tilde{d}_{2}=d_{4}+d_{5}$. By Lemma 2.1 (cf. also ACS3, p. 10]) the components $\check{r}_{1}, \check{r}_{2}$ of Ricci tensor $\check{r}$ of a $G$-invariant metric $\check{g}=\left.y_{1} B\right|_{\mathfrak{p}_{1}}+\left.y_{2} B\right|_{\mathfrak{p}_{2}}$ are given by

$$
\left\{\begin{array}{l}
\check{r}_{1}=\frac{1}{2 y_{1}}-\frac{y_{2}}{2 \tilde{d}_{1} y_{1}^{2}}\left[\left[\begin{array}{c}
2 \\
11
\end{array}\right]\right] \\
\check{r}_{2}=\frac{1}{2 y_{2}}-\frac{1}{2 \tilde{d}_{2} y_{2}}\left[\left[\begin{array}{c}
2 \\
11
\end{array}\right]\right]+\frac{y_{2}}{4 \tilde{d}_{2} y_{1}^{2}}\left[\left[\begin{array}{c}
2 \\
11
\end{array}\right]\right],
\end{array}\right.
$$

where $\left[\left[\begin{array}{c}2 \\ 11\end{array}\right]\right]=\frac{\tilde{d}_{1} \tilde{d}_{2}}{\tilde{d}_{1}+4 \tilde{d}_{2}}$.

Note that, in the notation of Lemma 2.3, we have that $r_{(1,1)}=r_{2}, r_{(1,2)}=r_{3}, r_{(2,1)}=r_{4}$ and $r_{(2,2)}=r_{5}$. From Lemma 2.3 we see that the horizontal part of $r_{(1,2)}\left(=r_{3}\right)$ equals to $\check{r}_{1}$ and the horizontal part of $r_{(2,1)}\left(=r_{4}\right)$ equals to $\check{r}_{2}$, and thus we get

$$
\left[\begin{array}{c}
5 \\
23
\end{array}\right]=d_{3} \frac{1}{\tilde{d}_{1}}\left[\left[\begin{array}{c}
2 \\
11
\end{array}\right]\right]=\frac{d_{3}\left(d_{4}+d_{5}\right)}{\left(d_{2}+d_{3}\right)+4\left(d_{4}+d_{5}\right)}, \quad\left[\begin{array}{c}
4 \\
22
\end{array}\right]=d_{4} \frac{1}{\tilde{d}_{2}}\left[\left[\begin{array}{c}
2 \\
11
\end{array}\right]\right]=\frac{d_{4}\left(d_{2}+d_{3}\right)}{\left(d_{2}+d_{3}\right)+4\left(d_{4}+d_{5}\right)} .
$$

We determine the structure constants $\left[\begin{array}{c}k \\ i j\end{array}\right]$ in each case.

Case of $B_{\ell}=\mathrm{SO}(2 \ell+1)$ : Type A.

In this case $G=\mathrm{SO}(2 \ell+1), K=\mathrm{U}(1) \times \mathrm{U}(p) \times \mathrm{SO}(2(\ell-p-1)+1), L=\mathrm{U}(p+1) \times \mathrm{SO}(2(\ell-p-1)+1)$ and we have $d_{1}=2 p, d_{2}=2 p(2 \ell-2 p-1), d_{3}=2(2 \ell-2 p-1), d_{4}=p(p-1), d_{5}=2 p$. Thus, from (36), we see that

$$
\left[\begin{array}{c}
5 \\
23
\end{array}\right]=\frac{(2 \ell-2 p-1) p}{2 \ell-1}, \quad\left[\begin{array}{c}
4 \\
22
\end{array}\right]=\frac{(2 \ell-2 p-1) p(p-1)}{2 \ell-1} .
$$

Since the Kähler Einstein metric $g_{2 \delta_{\mathfrak{n}}}$ on $G / K$ is given by

$$
g_{2 \delta_{\mathfrak{n}}}=\left.(p+1) B\right|_{\mathfrak{n}_{1}}+\left.(2 \ell-p-2) B\right|_{\mathfrak{n}_{2}}+\left.(2 \ell-1) B\right|_{\mathfrak{n}_{3}}+\left.2(2 \ell-p-2) B\right|_{\mathfrak{n}_{4}}+\left.(4 \ell-p-3) B\right|_{\mathfrak{n}_{5}},
$$

we substitute the values $x_{1}=p+1, x_{2}=2 \ell-p-2, x_{3}=2 \ell-1, x_{4}=2(2 \ell-p-2), x_{5}=4 \ell-p-3$ into (32). Consider the components $r_{2}, r_{3}, r_{4}$ and $r_{5}$ of the Ricci tensor for these values. Then, from $r_{2}-r_{3}=0$ and $r_{4}-r_{5}=0$, we obtain

$$
\left[\begin{array}{c}
3 \\
12
\end{array}\right]=\frac{(2 \ell-2 p-1) p}{2 \ell-1}, \quad\left[\begin{array}{c}
5 \\
14
\end{array}\right]=\frac{p(p-1)}{2 \ell-1} .
$$

Case of $D_{\ell}=\mathrm{SO}(2 \ell)$ : Type A.

In this case $G=\mathrm{SO}(2 \ell), K=\mathrm{U}(1) \times \mathrm{U}(p) \times \mathrm{SO}(2(\ell-p-1)), L=\mathrm{U}(p+1) \times \mathrm{SO}(2(\ell-p-1))$ and we have $d_{1}=2 p, d_{2}=4 p(\ell-p-1), d_{3}=4(\ell-p-1), d_{4}=p(p-1), d_{5}=2 p$. Thus, from (36), we see that

$$
\left[\begin{array}{c}
5 \\
23
\end{array}\right]=\frac{(\ell-p-1) p}{\ell-1}, \quad\left[\begin{array}{c}
4 \\
22
\end{array}\right]=\frac{(\ell-p-1) p(p-1)}{\ell-1} .
$$

Since the Kähler Einstein metric $g_{2 \delta_{\mathfrak{n}}}$ on $G / K$ is given by

$$
g_{2 \delta_{\mathfrak{n}}}=\left.(p+1) B\right|_{\mathfrak{n}_{1}}+\left.(2 \ell-p-3) B\right|_{\mathfrak{n}_{2}}+\left.(2 \ell-2) B\right|_{\mathfrak{n}_{3}}+\left.2(2 \ell-p-3) B\right|_{\mathfrak{n}_{4}}+\left.(4 \ell-p-5) B\right|_{\mathfrak{n}_{5}},
$$


we substitute the values $x_{1}=p+1, x_{2}=2 \ell-p-3, x_{3}=2 \ell-2, x_{4}=2(2 \ell-p-3), x_{5}=4 \ell-p-5$ into (32). Consider the components $r_{2}, r_{3}, r_{4}$ and $r_{5}$ of the Ricci tensor for these values. Then, from $r_{2}-r_{3}=0$ and $r_{4}-r_{5}=0$, we obtain

$$
\left[\begin{array}{c}
3 \\
12
\end{array}\right]=\frac{(\ell-p-1) p}{\ell-1}, \quad\left[\begin{array}{c}
5 \\
14
\end{array}\right]=\frac{p(p-1)}{2(\ell-1)}
$$

Note that we can put the cases of $B_{\ell}$ and $D_{\ell}$ together. Consider $G=\mathrm{SO}(m)$ and $K=\mathrm{U}(1) \times \mathrm{U}(p) \times$ $\mathrm{SO}(m-2(p+1))$. Then we have $d_{1}=2 p, d_{2}=2 p(m-2(p+1)), d_{3}=2(m-2(p+1)), d_{4}=p(p-1), d_{5}=2 p$ thus it follows that

$$
\left[\begin{array}{c}
5 \\
23
\end{array}\right]=\frac{(m-2(p+1)) p}{m-2}, \quad\left[\begin{array}{c}
4 \\
22
\end{array}\right]=\frac{(m-2(p+1)) p(p-1)}{m-2}
$$

and

$$
\left[\begin{array}{c}
3 \\
12
\end{array}\right]=\frac{(m-2(p+1)) p}{m-2}, \quad\left[\begin{array}{c}
5 \\
14
\end{array}\right]=\frac{p(p-1)}{m-2} .
$$

Case of $\mathrm{E}_{6}:$ Type A.

In this case $G=\mathrm{E}_{6}, K=\mathrm{U}(1) \times \mathrm{U}(1) \times \mathrm{SU}(2) \times \mathrm{SU}(4), L=\mathrm{U}(5) \times \mathrm{SU}(2)$ and we have $d_{1}=8, d_{2}=$ $24, d_{3}=16, d_{4}=2, d_{5}=8$. Thus, from (136), we see that

$$
\left[\begin{array}{c}
5 \\
23
\end{array}\right]=2, \quad\left[\begin{array}{c}
4 \\
22
\end{array}\right]=1 .
$$

Since the Kähler Einstein metric $g_{2 \delta_{\mathfrak{n}}}$ on $G / K$ is given by

$$
g_{2 \delta_{\mathfrak{n}}}=\left.5 B\right|_{\mathfrak{n}_{1}}+\left.7 B\right|_{\mathfrak{n}_{2}}+\left.12 B\right|_{\mathfrak{n}_{3}}+\left.14 B\right|_{\mathfrak{n}_{4}}+\left.19 B\right|_{\mathfrak{n}_{5}},
$$

we substitute the values $x_{1}=5, x_{2}=7, x_{3}=12, x_{4}=14, x_{5}=19$ into (32). Consider the components $r_{2}$, $r_{3}, r_{4}$ and $r_{5}$ of the Ricci tensor for these values. Then, from $r_{2}-r_{3}=0$ and $r_{4}-r_{5}=0$, we obtain

$$
\left[\begin{array}{c}
3 \\
12
\end{array}\right]=2, \quad\left[\begin{array}{c}
5 \\
14
\end{array}\right]=\frac{1}{3} \text {. }
$$

Case of $\mathrm{E}_{7}:$ Type A.

In this case $G=\mathrm{E}_{7}, K=\mathrm{U}(1) \times \mathrm{U}(1) \times \mathrm{SU}(6), L=\mathrm{U}(7)$ and we have $d_{1}=12, d_{2}=40, d_{3}=30, d_{4}=$ $2, d_{5}=12$. Thus, from (36), we see that

$$
\left[\begin{array}{c}
5 \\
23
\end{array}\right]=\frac{10}{3}, \quad\left[\begin{array}{c}
4 \\
22
\end{array}\right]=\frac{10}{9} .
$$

Since the Kähler Einstein metric $g_{2 \delta_{\mathfrak{n}}}$ on $G / K$ is given by

$$
g_{2 \delta_{\mathfrak{n}}}=\left.7 B\right|_{\mathfrak{n}_{1}}+\left.11 B\right|_{\mathfrak{n}_{2}}+\left.18 B\right|_{\mathfrak{n}_{3}}+\left.22 B\right|_{\mathfrak{n}_{4}}+\left.29 B\right|_{\mathfrak{n}_{5}},
$$

we substitute the values $x_{1}=7, x_{2}=11, x_{3}=18, x_{4}=22, x_{5}=29$ into (32). Consider the components $r_{2}$, $r_{3}, r_{4}$ and $r_{5}$ of the Ricci tensor for these values. Then, from $r_{2}-r_{3}=0$ and $r_{4}-r_{5}=0$, we obtain

$$
\left[\begin{array}{c}
3 \\
12
\end{array}\right]=\frac{10}{3}, \quad\left[\begin{array}{c}
5 \\
14
\end{array}\right]=\frac{1}{3} \text {. }
$$

\section{EINSTEIN METRICS ON FLAG MANIFOLDS WITH FIVE ISOTROPY SUMMNADS}

We consider the system of equations:

$$
r_{1}=r_{5}, \quad r_{2}=r_{3}, \quad r_{3}=r_{4}, \quad r_{4}=r_{5} .
$$

Case of $\mathrm{E}_{6}:$ Type A. 
The components $r_{i}(i=1, \ldots, 5)$ of the Ricci tensor for a $G$-invariant Riemannian metric (31) on $G / K$ are now given as follows:

$$
\begin{aligned}
& r_{1}=\frac{1}{2 x_{1}}+\frac{1}{8}\left(\frac{x_{1}}{x_{2} x_{3}}-\frac{x_{2}}{x_{1} x_{3}}-\frac{x_{3}}{x_{1} x_{2}}\right)+\frac{1}{48}\left(\frac{x_{1}}{x_{4} x_{5}}-\frac{x_{5}}{x_{1} x_{4}}-\frac{x_{4}}{x_{1} x_{5}}\right), \\
& r_{2}=\frac{1}{2 x_{2}}+\frac{1}{24}\left(\frac{x_{2}}{x_{1} x_{3}}-\frac{x_{1}}{x_{2} x_{3}}-\frac{x_{3}}{x_{1} x_{2}}\right)-\frac{1}{48} \frac{x_{4}}{x_{2}^{2}}+\frac{1}{24}\left(\frac{x_{2}}{x_{3} x_{5}}-\frac{x_{5}}{x_{2} x_{3}}-\frac{x_{3}}{x_{2} x_{5}}\right), \\
& r_{3}=\frac{1}{2 x_{3}}+\frac{1}{16}\left(\frac{x_{3}}{x_{1} x_{2}}-\frac{x_{2}}{x_{1} x_{3}}-\frac{x_{1}}{x_{2} x_{3}}\right)+\frac{1}{16}\left(\frac{x_{3}}{x_{2} x_{5}}-\frac{x_{5}}{x_{2} x_{3}}-\frac{x_{2}}{x_{3} x_{5}}\right), \\
& r_{4}=\frac{1}{2 x_{4}}+\frac{1}{12}\left(\frac{x_{4}}{x_{1} x_{5}}-\frac{x_{5}}{x_{1} x_{4}}-\frac{x_{1}}{x_{4} x_{5}}\right)+\frac{1}{8}\left(-\frac{2}{x_{4}}+\frac{x_{4}}{x_{2}^{2}}\right), \\
& r_{5}=\frac{1}{2 x_{5}}+\frac{1}{8}\left(\frac{x_{5}}{x_{2} x_{3}}-\frac{x_{2}}{x_{3} x_{5}}-\frac{x_{3}}{x_{2} x_{5}}\right)+\frac{1}{48}\left(\frac{x_{5}}{x_{1} x_{4}}-\frac{x_{1}}{x_{4} x_{5}}-\frac{x_{4}}{x_{1} x_{5}}\right) .
\end{aligned}
$$

From $r_{1}-r_{5}=0$, we see that

$$
\left(x_{1}-x_{5}\right)\left(x_{1} x_{2} x_{3}+3 x_{1} x_{4} x_{5}+3 x_{2}^{2} x_{4}-12 x_{2} x_{3} x_{4}+x_{2} x_{3} x_{5}+3 x_{3}^{2} x_{4}\right)=0 .
$$

Case of $x_{5}=x_{1}$. We normalize our equations by setting $x_{1}=1$. We see that the system of equations (37) reduces to the following system of polynomial equations:

$$
\left.\begin{array}{l}
f_{1}=10 x_{2}^{3}+x_{2}^{2} x_{3} x_{4}-24 x_{2}^{2} x_{3}+2 x_{2} x_{3}^{2}+24 x_{2} x_{3}-10 x_{2}-x_{3} x_{4}=0, \\
f_{2}=10 x_{2}^{3}-24 x_{2}^{2}-10 x_{2} x_{3}^{2}+24 x_{2} x_{3}+2 x_{2}-x_{3} x_{4}=0, \\
f_{3}=3 x_{2}{ }^{3} x_{4}+2 x_{2}{ }^{2} x_{3} x_{4}{ }^{2}+2 x_{2}^{2} x_{3}-12 x_{2}^{2} x_{4}-3 x_{2} x_{3}^{2} x_{4}+3 x_{2} x_{4}+3 x_{3} x_{4}{ }^{2}=0
\end{array}\right\}
$$

To find non zero solutions of equations (39) we consider a polynomial ring $R_{1}=\mathbb{Q}\left[y, x_{2}, x_{3}, x_{4}\right]$ and an ideal $I_{1}$ generated by

$$
\left\{f_{1}, f_{2}, f_{3}, y x_{2} x_{3} x_{4}-1\right\} .
$$

We take a lexicographic order $>$ with $y>x_{2}>x_{3}>x_{4}$ for a monomial ordering on $R_{1}$. Then a Gröbner basis for the ideal $I_{1}$ contains the following polynomials:

$$
\begin{aligned}
& h_{1}=512683897 x_{4}{ }^{12}-26586224544 x_{4}{ }^{11}+613729012600 x_{4}{ }^{10}-8672203136256 x_{4}{ }^{9} \\
& +79425819414800 x_{4}{ }^{8}-364553102019072 x_{4}{ }^{7}+901989582472192 x_{4}{ }^{6} \\
& -1275600747577344 x_{4}{ }^{5}+1046901453080576 x_{4}{ }^{4}-491806714331136 x_{4}{ }^{3} \\
& +129330076549120 x_{4}{ }^{2}-17647691366400 x_{4}+969515008000, \\
& h_{2}=114848188839160612119624999242582277039963322780084212652611305472000 x_{3} \\
& -752320404408788199702048033270865700909380495228817968360883312339 x_{4}{ }^{11} \\
& +38758220515867322791999260031297235508730394711323449754223470870360 x_{4}{ }^{10}+\cdots \\
& -70726659216761168399944465106568342085848237958573454324691582101708800 x_{4} \\
& +4794499893690636543924823161512975441415943523673600438772484784128000, \\
& h_{3}=86136141629370459089718749431936707779972492085063159489458479104000 x_{2} \\
& +523691563864872091386883937890449783253444913328748812397494319729 x_{4}{ }^{11} \\
& -27032170704374631808506232904135459706304200757258747894928587117499 x_{4}{ }^{10}+\cdots \\
& +52958437343374493285824611500843861525218627552705339462466929126522880 x_{4} \\
& -3633544639518951458129167566718404600177262066660959706336805062451200 .
\end{aligned}
$$

By solving the first equation $h_{1}=0$ for $x_{4}$ numerically we obtain exactly four real solutions which are approximately given by $x_{4} \approx 0.1882101376884833, x_{4} \approx 0.3421847475947193, x_{4} \approx 1.334632880397468$ and $x_{4} \approx 1.601718258421132$. We substitute these values for $x_{4}$ into the second and third equation $h_{2}=0$, 
$h_{3}=0$ and we get real solutions of the equations (37) which are approximately given by
1) $x_{1}=1, x_{2} \approx 0.7945133013133368, x_{3} \approx 0.6083856170340604, x_{4} \approx 0.1882101376884833, x_{5}=1$,
2) $x_{1}=1, x_{2} \approx 1.366407998279779, x_{3} \approx 1.632222678282746, x_{4} \approx 0.3421847475947193, x_{5}=1$,
3) $x_{1}=1, x_{2} \approx 0.7499994899122792, x_{3} \approx 0.6673176327222041, x_{4} \approx 1.334632880397468, x_{5}=1$,
4) $x_{1}=1, x_{2} \approx 1.590451006762520, x_{3} \approx 1.633523267052982, x_{4} \approx 1.601718258421132, x_{5}=1$.

We substitute these values for $\left\{x_{1}, x_{2}, x_{3}, x_{4}, x_{5}\right\}$ into (38) and get
1) $r_{1}=r_{2}=r_{3}=r_{4}=r_{5} \approx 0.4957209368544092$,
2) $r_{1}=r_{2}=r_{3}=r_{4}=r_{5} \approx 0.2949577540873313$,
3) $r_{1}=r_{2}=r_{3}=r_{4}=r_{5} \approx 0.4702440377042893$,
4) $r_{1}=r_{2}=r_{3}=r_{4}=r_{5} \approx 0.2646548256739946$.

Thus, in this case we obtain four Einstein metrics with Einstein constant 1:

1) $x_{1} \approx 0.49572094, x_{2} \approx 0.39385688, x_{3} \approx 0.30158949, x_{4} \approx 0.093299706, x_{5} \approx 0.49572094$,

2) $\quad x_{1} \approx 0.29495775, x_{2} \approx 0.40303263, x_{3} \approx 0.48143674, x_{4} \approx 0.10093004, x_{5} \approx 0.29495775$,

3) $x_{1} \approx 0.47024404, x_{2} \approx 0.35268279, x_{3} \approx 0.31380214, x_{4} \approx 0.62760315, x_{5} \approx 0.47024404$,

4) $x_{1} \approx 0.26465483, x_{2} \approx 0.42092053, x_{3} \approx 0.43231982, x_{4} \approx 0.42390247, x_{5} \approx 0.26465483$.

Case of $x_{5} \neq x_{1}$. We normalize our equations by setting $x_{1}=1$. We see that the system of polynomial equations (37) reduces to the following system of polynomial equations:

$$
\left.\begin{array}{l}
p_{1}=-8 x_{2}{ }^{3} x_{4} x_{5}-2 x_{2}{ }^{3} x_{4}-x_{2}{ }^{2} x_{3} x_{4}{ }^{2}+24 x_{2}{ }^{2} x_{3} x_{4} x_{5}-x_{2}{ }^{2} x_{3} x_{5}{ }^{2}+x_{2}{ }^{2} x_{3}-4 x_{2} x_{3}{ }^{2} x_{4} x_{5} \\
+2 x_{2} x_{3}{ }^{2} x_{4}-24 x_{2} x_{3} x_{4} x_{5}+2 x_{2} x_{4} x_{5}{ }^{2}+8 x_{2} x_{4} x_{5}+x_{3} x_{4}{ }^{2} x_{5}=0, \\
p_{2}=5 x_{2}{ }^{3} x_{5}+5 x_{2}{ }^{3}-24 x_{2}^{2} x_{5}-5 x_{2} x_{3}{ }^{2} x_{5}-5 x_{2} x_{3}{ }^{2}+24 x_{2} x_{3} x_{5}+x_{2} x_{5}{ }^{2}+x_{2} x_{5}-x_{3} x_{4} x_{5}=0, \\
p_{3}=-3 x_{2}{ }^{3} x_{4} x_{5}-3 x_{2}{ }^{3} x_{4}-4 x_{2}{ }^{2} x_{3} x_{4}{ }^{2}+4 x_{2}{ }^{2} x_{3} x_{5}{ }^{2}-12 x_{2}{ }^{2} x_{3} x_{5}+4 x_{2}{ }^{2} x_{3}+24 x_{2}{ }^{2} x_{4} x_{5} \\
+3 x_{2} x_{3}{ }^{2} x_{4} x_{5}+3 x_{2} x_{3}{ }^{2} x_{4}-3 x_{2} x_{4} x_{5}{ }^{2}-3 x_{2} x_{4} x_{5}-6 x_{3} x_{4}{ }^{2} x_{5}=0, \\
p_{4}=3 x_{2}{ }^{2} x_{4}-12 x_{2} x_{3} x_{4}+x_{2} x_{3} x_{5}+x_{2} x_{3}+3 x_{3}{ }^{2} x_{4}+3 x_{4} x_{5}=0 .
\end{array}\right\}
$$

To find non zero solutions of equations (40), we consider a polynomial ring $R_{2}=\mathbb{Q}\left[y, x_{2}, x_{3}, x_{4}, x_{5}\right]$ and an ideal $I_{2}$ generated by

$$
\left\{p_{1}, p_{2}, p_{3}, p_{4}, y x_{2} x_{3} x_{4} x_{5}-1\right\} .
$$

We take a lexicographic order $>$ with $y>x_{2}>x_{5}>x_{3}>x_{4}$ for a monomial ordering on $R_{2}$. Then a Gröbner basis for the ideal $I_{2}$ contains a polynomial

$$
\left(5 x_{4}-22\right)\left(5 x_{4}-14\right)\left(17 x_{4}-22\right)\left(19 x_{4}-14\right) q_{1},
$$

where

$$
\begin{aligned}
& q_{1}=25684944948354308203125 x_{4}{ }^{24}-312330714783423219879187500 x_{4}{ }^{23} \\
& -14789576030598686784365775000 x_{4}{ }^{22}+169312435225853499159893370000 x_{4}{ }^{21}+\cdots \\
& -597859726821790689492624998400 x_{4}{ }^{4}+84059799581674625557541683200 x_{4}{ }^{3} \\
& -2979131989754489205686272000 x_{4}{ }^{2}-1842910805533143334912000000 x_{4} \\
& +333622121893933875200000000 .
\end{aligned}
$$

For the case when $\left(5 x_{4}-22\right)\left(5 x_{4}-14\right)\left(17 x_{4}-22\right)\left(19 x_{4}-14\right)=0$, we consider ideals $I_{3}, I_{4}, I_{5}, I_{6}$ of the polynomial ring $R_{2}=\mathbb{Q}\left[y, x_{2}, x_{3}, x_{4}, x_{5}\right]$ generated by

$\left\{p_{1}, p_{2}, p_{3}, p_{4}, y, x_{2} x_{3} x_{4} x_{5}-1,5 x_{4}-22\right\}, \quad\left\{p_{1}, p_{2}, p_{3}, p_{4}, y, x_{2} x_{3} x_{4} x_{5}-1,5 x_{4}-14\right\}$,

$\left\{p_{1}, p_{2}, p_{3}, p_{4}, y, x_{2} x_{3} x_{4} x_{5}-1,17 x_{4}-22\right\}, \quad\left\{p_{1}, p_{2}, p_{3}, p_{4}, y, x_{2} x_{3} x_{4} x_{5}-1,17 x_{4}-14\right\}$

respectively.

We take a lexicographic order $>$ with $y>x_{2}>x_{5}>x_{3}>x_{4}$ for a monomial ordering on $R_{2}$. Then Gröbner bases for the ideals $I_{3}, I_{4}, I_{5}, I_{6}$ contain polynomials

$$
\begin{array}{r}
\left\{5 x_{4}-22,5 x_{3}-6,5 x_{5}-17,5 x_{2}-11\right\}, \quad\left\{5 x_{4}-14,5 x_{3}-12,5 x_{5}-19,5 x_{2}-7\right\}, \\
\left\{17 x_{4}-22,17 x_{3}-6,17 x_{5}-5,17 x_{2}-11\right\}, \quad\left\{19 x_{4}-14,19 x_{3}-12,19 x_{5}-5,19 x_{2}-7\right\} .
\end{array}
$$

respectively. Thus we obtain the following solutions of equations (40): 

1) $x_{1}=1, x_{2}=\frac{11}{5}, x_{3}=\frac{6}{5}, x_{4}=\frac{22}{5}, x_{5}=\frac{17}{5}$
2) $x_{1}=1, x_{2}=\frac{7}{5}, x_{3}=\frac{12}{5}, x_{4}=\frac{14}{5}, x_{5}=\frac{19}{5}$,
3) $x_{1}=1, x_{2}=\frac{11}{17}, x_{3}=\frac{6}{17}, x_{4}=\frac{22}{17}, x_{5}=\frac{5}{17}$,
4) $x_{1}=1, x_{2}=\frac{7}{19}, x_{3}=\frac{12}{19}, x_{4}=\frac{14}{19}, x_{5}=\frac{5}{19}$.

We normalize these solutions as follows:
1) $x_{1}=5, x_{2}=11, x_{3}=6, x_{4}=22, x_{5}=17$,
2) $x_{1}=5, x_{2}=7, x_{3}=12, x_{4}=14, x_{5}=19$,
3) $x_{1}=17, x_{2}=11, x_{3}=6, x_{4}=22, x_{5}=5$,
4) $x_{1}=19, x_{2}=7, x_{3}=12, x_{4}=14, x_{5}=5$.

and we get Kähler Einstein metrics for these values of $x_{i}$ 's. Note that the metrics corresponding to the cases 1) and 3) are isometric and the cases 2) and 4) are isometric.

For the case when $q_{1}=0$ and $\left(5 x_{4}-22\right)\left(5 x_{4}-14\right)\left(17 x_{4}-22\right)\left(19 x_{4}-14\right) \neq 0$, we consider a ideal $I_{7}$ of the polynomial ring $R_{2}=\mathbb{Q}\left[y, x_{2}, x_{3}, x_{4}, x_{5}\right]$ generated by

$$
\left\{p_{1}, p_{2}, p_{3}, p_{4}, y\left(5 x_{4}-22\right)\left(5 x_{4}-14\right)\left(17 x_{4}-22\right)\left(19 x_{4}-14\right) x_{2} x_{3} x_{4} x_{5}-1\right\} \text {. }
$$

We take the same lexicographic order $>$ with $y>x_{2}>x_{5}>x_{3}>x_{4}$ for a monomial ordering on $R_{2}$. Then a Gröbner basis for the ideal $I_{7}$ contains the polynomial $q_{1}$ and polynomials of the form

$$
b_{2} x_{2}+v_{2}\left(x_{4}\right), \quad b_{3} x_{3}+v_{3}\left(x_{4}\right), \quad b_{5} x_{5}+v_{5}\left(x_{4}\right)
$$

where $b_{2}, b_{3}, b_{5}$ are positive integers and $v_{2}\left(x_{4}\right), v_{3}\left(x_{4}\right), v_{5}\left(x_{4}\right)$ are polynomials of degree 23 with integer coefficients.

By solving the equation $q_{1}=0$ for $x_{4}$ numerically, we obtain exactly 6 positive solutions, 8 negative solutions and 10 non-real solutions. The 6 positive solutions are approximately given by

$$
\begin{array}{lll}
\text { 1) } x_{4} \approx 1.157018562397866, & \text { 2) } x_{4} \approx 2.075646788197390, & \text { 3) } x_{4} \approx 2.145057741729789, \\
\text { 4) } x_{4} \approx 2.163849575049888, & \text { 5) } x_{4} \approx 12.97930323340096, & \text { 6) } x_{4} \approx 12207.19468694106 .
\end{array}
$$

We substitute the values for $x_{4}$ into the equations $b_{2} x_{2}+v_{2}\left(x_{4}\right)=0, b_{3} x_{3}+v_{3}\left(x_{4}\right)=0, b_{5} x_{5}+v_{5}\left(x_{4}\right)=0$. Then we obtain the following values approximately:

$$
\begin{aligned}
& \text { 1) } x_{4} \approx 1.15702, x_{2} \approx 0.641194, x_{3} \approx 0.566074, x_{5} \approx 0.557426, \\
& \text { 2) } x_{4} \approx 2.07565, x_{2} \approx 1.15028, x_{3} \approx 1.01551, x_{5} \approx 1.79396, \\
& \text { 3) } x_{4} \approx 2.14506, x_{2} \approx 8.87367, x_{3} \approx 33.3409, x_{5} \approx-1.12628, \\
& \text { 4) } x_{4} \approx 2.16385, x_{2} \approx 27.3523, x_{3} \approx 7.26471, x_{5} \approx-1.16127, \\
& \text { 5) } x_{4} \approx 12.9793, x_{2} \approx 1.3699, x_{3} \approx 5.42602, x_{5} \approx-1.49194, \\
& \text { 6) } x_{4} \approx 12207.2, x_{2} \approx 18.0447, x_{3} \approx 1.46532, x_{5} \approx-221.833 .
\end{aligned}
$$

Thus we see that only the cases 1) and 2) correspond to Einstein metrics. We substitute these values for $\left\{x_{1}, x_{2}, x_{3}, x_{4}, x_{5}\right\}$ into (38) and get

$$
\text { 1) } r_{1}=r_{2}=r_{3}=r_{4}=r_{5} \approx 0.31855, \quad \text { 2) } r_{1}=r_{2}=r_{3}=r_{4}=r_{5} \approx 0.571467 \text {. }
$$

Thus we obtain two Einstein metrics with Einstein constant 1:

1) $x_{1} \approx 0.31855, x_{2} \approx 0.366421, x_{3} \approx 0.323492, x_{4} \approx 0.661198, x_{5} \approx 0.571467$,

2) $x_{1} \approx 0.571467, x_{2} \approx 0.366421, x_{3} \approx 0.323492, x_{4} \approx 0.661198, x_{5} \approx 0.31855$.

Now we see that these two metrics are isometric.

Theorem 7.1. The flag manifold $\mathrm{E}_{6} /(\mathrm{SU}(4) \times \mathrm{SU}(2) \times \mathrm{U}(1) \times \mathrm{U}(1))$ admits exactly seven $\mathrm{E}_{6}$-invariant Einstein metrics up to isometry. There are two Kähler-Einstein metrics (up to scalar) given by

$$
\left\{x_{1}=5, x_{2}=7, x_{3}=12, x_{4}=14, x_{5}=19\right\} \quad\left\{x_{1}=5, x_{2}=11, x_{3}=6, x_{4}=22, x_{5}=17\right\} .
$$


The other five are non-Kähler. These metrics are given approximately by

$$
\begin{aligned}
& \left\{x_{1} \approx 0.571467, x_{2} \approx 0.366421, x_{3} \approx 0.323492, x_{4} \approx 0.661198, x_{5} \approx 0.31855\right\} \\
& \left\{x_{1} \approx 0.49572094, x_{2} \approx 0.39385688, x_{3} \approx 0.30158949, x_{4} \approx 0.093299706, x_{5} \approx 0.49572094\right\} \\
& \left\{x_{1} \approx 0.29495775, x_{2} \approx 0.40303263, x_{3} \approx 0.48143674, x_{4} \approx 0.10093004, x_{5} \approx 0.29495775\right\} \\
& \left\{x_{1} \approx 0.47024404, x_{2} \approx 0.35268279, x_{3} \approx 0.31380214, x_{4} \approx 0.62760315, x_{5} \approx 0.47024404\right\} \\
& \left\{x_{1} \approx 0.26465483, x_{2} \approx 0.42092053, x_{3} \approx 0.43231982, x_{4} \approx 0.42390247, x_{5} \approx 0.26465483\right\}
\end{aligned}
$$

\section{Case of $\mathrm{E}_{7}:$ Type A.}

The components $r_{i}(i=1, \cdots, 5)$ of the Ricci tensor for a $G$-invariant Riemannian metric (31) on $G / K$ are given as follows:

$$
\begin{aligned}
& r_{1}=\frac{1}{2 x_{1}}+\frac{5}{36}\left(\frac{x_{1}}{x_{2} x_{3}}-\frac{x_{2}}{x_{1} x_{3}}-\frac{x_{3}}{x_{1} x_{2}}\right)+\frac{1}{72}\left(\frac{x_{1}}{x_{4} x_{5}}-\frac{x_{5}}{x_{1} x_{4}}-\frac{x_{4}}{x_{1} x_{5}}\right) \\
& r_{2}=\frac{1}{2 x_{2}}+\frac{1}{24}\left(\frac{x_{2}}{x_{1} x_{3}}-\frac{x_{1}}{x_{2} x_{3}}-\frac{x_{3}}{x_{1} x_{2}}\right)-\frac{1}{72} \frac{x_{4}}{x_{2}^{2}}+\frac{1}{24}\left(\frac{x_{2}}{x_{3} x_{5}}-\frac{x_{5}}{x_{2} x_{3}}-\frac{x_{3}}{x_{2} x_{5}}\right) \\
& r_{3}=\frac{1}{2 x_{3}}+\frac{1}{18}\left(\frac{x_{3}}{x_{1} x_{2}}-\frac{x_{2}}{x_{1} x_{3}}-\frac{x_{1}}{x_{2} x_{3}}\right)+\frac{1}{18}\left(\frac{x_{3}}{x_{2} x_{5}}-\frac{x_{5}}{x_{2} x_{3}}-\frac{x_{2}}{x_{3} x_{5}}\right) \\
& r_{4}=\frac{1}{2 x_{4}}+\frac{1}{12}\left(\frac{x_{4}}{x_{1} x_{5}}-\frac{x_{5}}{x_{1} x_{4}}-\frac{x_{1}}{x_{4} x_{5}}\right)+\frac{5}{36}\left(-\frac{2}{x_{4}}+\frac{x_{4}}{x_{2} 2}\right), \\
& r_{5}=\frac{1}{2 x_{5}}+\frac{5}{36}\left(\frac{x_{5}}{x_{2} x_{3}}-\frac{x_{2}}{x_{3} x_{5}}-\frac{x_{3}}{x_{2} x_{5}}\right)+\frac{1}{72}\left(\frac{x_{5}}{x_{1} x_{4}}-\frac{x_{1}}{x_{4} x_{5}}-\frac{x_{4}}{x_{1} x_{5}}\right) .
\end{aligned}
$$

By using similar method as for the case of $\mathrm{E}_{6}$ we end up to the following:

Theorem 7.2. The flag manifold $\mathrm{E}_{7} /(\mathrm{U}(1) \times \mathrm{U}(6))$ admits exactly seven $\mathrm{E}_{7}$-invariant Einstein metrics up to isometry. There are two Kähler-Einstein metrics (up to scalar) given by

$$
\left\{x_{1}=7, x_{2}=11, x_{3}=18, x_{4}=22, x_{5}=29\right\}, \quad\left\{x_{1}=7, x_{2}=17, x_{3}=10, x_{4}=34, x_{5}=27\right\} .
$$

The other five are non-Kähler. These metrics are given approximately by

$$
\begin{aligned}
& \left\{x_{1} \approx 0.63931715, x_{2} \approx 0.37800271, x_{3} \approx 0.34993635, x_{4} \approx 0.69900421, x_{5} \approx 0.27564786\right\} \\
& \left\{x_{1} \approx 0.52602201, x_{2} \approx 0.38291429, x_{3} \approx 0.32460549, x_{4} \approx 0.060058655, x_{5} \approx 0.52602201\right\} \\
& \left\{x_{1} \approx 0.26773609, x_{2} \approx 0.42433469, x_{3} \approx 0.46801223, x_{4} \approx 0.063305828, x_{5} \approx 0.2677360\right\} \\
& \left\{x_{1} \approx 0.50711535, x_{2} \approx 0.35565283, x_{3} \approx 0.33123840, x_{4} \approx 0.64238182, x_{5} \approx 0.50711535\right\} \\
& \left\{x_{1} \approx 0.24046904, x_{2} \approx 0.43874160, x_{3} \approx 0.44384361, x_{4} \approx 0.39782398, x_{5} \approx 0.24046904\right\}
\end{aligned}
$$

Now we consider the cases of $B_{\ell}=\mathrm{SO}(2 \ell+1)$ and $D_{\ell}=\mathrm{SO}(2 \ell)$ together.

Case of $\mathrm{SO}(m)$ : Type A.

The components $r_{i}(i=1, \ldots, 5)$ of the Ricci tensor for a $G$-invariant Riemannian metric (31) on $G / K=\mathrm{SO}(m) /(\mathrm{U}(1) \times \mathrm{U}(p) \times \mathrm{SO}(m-2-2 p))$ are now given as follows:

$$
\left.\begin{array}{l}
r_{1}=\frac{1}{2 x_{1}}+\frac{m-2-2 p}{4(m-2)}\left(\frac{x_{1}}{x_{2} x_{3}}-\frac{x_{2}}{x_{1} x_{3}}-\frac{x_{3}}{x_{1} x_{2}}\right)+\frac{p-1}{4(m-2)}\left(\frac{x_{1}}{x_{4} x_{5}}-\frac{x_{5}}{x_{1} x_{4}}-\frac{x_{4}}{x_{1} x_{5}}\right), \\
r_{2}=\frac{1}{2 x_{2}}+\frac{1}{4(m-2)}\left(\frac{x_{2}}{x_{1} x_{3}}-\frac{x_{1}}{x_{2} x_{3}}-\frac{x_{3}}{x_{1} x_{2}}\right)-\frac{p-1}{4(m-2)} \frac{x_{4}}{x_{2}^{2}}+\frac{1}{4(m-2)}\left(\frac{x_{2}}{x_{3} x_{5}}-\frac{x_{5}}{x_{2} x_{3}}-\frac{x_{3}}{x_{2} x_{5}}\right), \\
r_{3}=\frac{1}{2 x_{3}}+\frac{p}{4(m-2)}\left(\frac{x_{3}}{x_{1} x_{2}}-\frac{x_{2}}{x_{1} x_{3}}-\frac{x_{1}}{x_{2} x_{3}}\right)+\frac{p}{4(m-2)}\left(\frac{x_{3}}{x_{2} x_{5}}-\frac{x_{5}}{x_{2} x_{3}}-\frac{x_{2}}{x_{3} x_{5}}\right), \\
r_{4}=\frac{1}{2 x_{4}}+\frac{1}{2(m-2)}\left(\frac{x_{4}}{x_{1} x_{5}}-\frac{x_{5}}{x_{1} x_{4}}-\frac{x_{1}}{x_{4} x_{5}}\right)+\frac{(m-2-2 p)}{4(m-2)}\left(-\frac{2}{x_{4}}+\frac{x_{4}}{x_{2}^{2}}\right), \\
r_{5}=\frac{1}{2 x_{5}}+\frac{m-2-2 p}{4(m-2)}\left(\frac{x_{5}}{x_{2} x_{3}}-\frac{x_{2}}{x_{3} x_{5}}-\frac{x_{3}}{x_{2} x_{5}}\right)+\frac{p-1}{4(m-2)}\left(\frac{x_{5}}{x_{1} x_{4}}-\frac{x_{1}}{x_{4} x_{5}}-\frac{x_{4}}{x_{1} x_{5}}\right) .
\end{array}\right\}
$$


From $r_{1}-r_{5}=0$, we see that

$$
\begin{aligned}
& \left(x_{1}-x_{5}\right)\left((m-2-2 p) x_{1} x_{4} x_{5}+(m-2-2 p) x_{2}^{2} x_{4}+(m-2-2 p) x_{3}{ }^{2} x_{4}\right. \\
& \left.-2(m-2) x_{2} x_{3} x_{4}+2(p-1) x_{1} x_{2} x_{3}+2(p-1) x_{2} x_{3} x_{5}\right)=0 .
\end{aligned}
$$

Case of $x_{5}=x_{1}$. We normalize our equations by setting $x_{1}=1$. We see that the system of polynomial equations (37) reduces to the following system of polynomial equations:

$$
\begin{aligned}
& f_{1}=-(m-2 p) x_{2}{ }^{3}-(m-4-2 p) x_{2} x_{3}{ }^{2}+(m-2 p) x_{2} \\
& +2(m-2) x_{2}{ }^{2} x_{3}-2(m-2) x_{2} x_{3}-(p-1) x_{2}{ }^{2} x_{3} x_{4}+(p-1) x_{3} x_{4}=0, \\
& f_{2}=-2(m-2) x_{2}{ }^{2}+2(m-2) x_{2} x_{3}+2(p+1) x_{2}{ }^{3}-2(p+1) x_{2} x_{3}{ }^{2} \\
& +2(p-1) x_{2}-(p-1) x_{3} x_{4}=0, \\
& f_{3}=-(m-2-2 p) x_{3} x_{4}{ }^{2}+2(m-2) x_{2}{ }^{2} x_{4}-2 p x_{2}{ }^{3} x_{4}-4(p-1) x_{2}{ }^{2} x_{3} \\
& +2 p x_{2} x_{3}{ }^{2} x_{4}-2 p x_{2} x_{4}-2 x_{2}{ }^{2} x_{3} x_{4}{ }^{2}=0 .
\end{aligned}
$$

To find non zero solutions of equations (45), we consider a polynomial ring $R=\mathbb{Q}\left[y, x_{2}, x_{3}, x_{4}\right]$ and an ideal $I_{1}$ generated by

$$
\left\{f_{1}, f_{2}, f_{3}, y x_{2} x_{3} x_{4}-1\right\} .
$$

We take a lexicographic order $>$ with $y>x_{2}>x_{4}>x_{3}$ for a monomial ordering on $R$. Then we see that a Gröbner basis for the ideal $I_{1}$ contains the following polynomial $h_{1}\left(x_{3}\right)$ of degree 12 :

$$
\begin{aligned}
& h_{1}\left(x_{3}\right)=16(p+1)^{5}\left(-p^{2}+m p-5 p+2 m-4\right)^{2}\left(p^{2}+4 p-1\right)\left(p^{3}+5 p^{2}-16 m p+35 p+8 m^{2}-32 m+31\right) x_{3}{ }^{12} \\
& -32(m-2)(p+1)^{4}\left(-p^{2}+m p-5 p+2 m-4\right)\left(-5 p^{7}+5 m p^{6}-62 p^{6}-m^{2} p^{5}+116 m p^{5}-403 p^{5}-88 m^{2} p^{4}\right. \\
& +863 m p^{4}-1562 p^{4}+24 m^{3} p^{3}-600 m^{2} p^{3}+2642 m p^{3}-3067 p^{3}+128 m^{3} p^{2}-1226 m^{2} p^{2}+3241 m p^{2}-2570 p^{2} \\
& \left.+128 m^{3} p-655 m^{2} p+1074 m p-557 p-24 m^{3}+138 m^{2}-261 m+162\right) x_{3}{ }^{11}+\cdots \\
& -4(m-2)(m-2 p-2)(m-2 p)(m-p-1)^{3}(m+2 p-2)\left(56 p^{7}-100 m p^{6}+272 p^{6}+58 m^{2} p^{5}-368 m p^{5}+448 p^{5}\right. \\
& -7 m^{3} p^{4}+64 m^{2} p^{4}-172 m p^{4}+72 p^{4}-4 m^{4} p^{3}+100 m^{3} p^{3}-408 m^{2} p^{3}+624 m p^{3}-352 p^{3}+m^{5} p^{2}-41 m^{4} p^{2} \\
& +253 m^{3} p^{2}-622 m^{2} p^{2}+696 m p^{2}-304 p^{2}-2 m^{5} p-m^{4} p+66 m^{3} p-212 m^{2} p+248 m p-96 p+2 m^{6}-15 m^{5} \\
& \left.+40 m^{4}-40 m^{3}+16 m\right) x_{3} \\
& +(m-2 p-2)^{2}(m-2 p)^{2}(m-p-1)^{4}(m+2 p-2)^{2}\left(4 p^{4}-4 m p^{3}+16 p^{3}+m^{2} p^{2}-8 m p^{2}+16 p^{2}-4 m^{2} p\right. \\
& \left.+16 m p-16 p+2 m^{3}-12 m^{2}+24 m-16\right)
\end{aligned}
$$

and polynomials of the form

$$
b_{2} x_{2}+v_{2}\left(x_{3}\right), \quad b_{3} x_{4}+v_{3}\left(x_{3}\right),
$$

where $b_{2}, b_{3}$ are integers depending on $m$ and $p$ and $v_{2}\left(x_{3}\right), v_{3}\left(x_{3}\right)$ are polynomials of degree 11 with integer coefficients depending on $m$ and $p$.

Note that for $2 \leq p \leq \frac{m-3}{2}$, we see that

$$
\begin{aligned}
& h_{1}(0)=(m-2 p-2)^{2}(m-2 p)^{2}(m-p-1)^{4}(m+2 p-2)^{2} \times \\
& \left(2(m-2 p-2)^{3}+\left(p^{2}+8 p\right)(m-2 p-2)^{2}+4 p^{2}(m-2 p-2)+4 p^{2}\right)>0
\end{aligned}
$$

and the head coefficient of $h_{1}\left(x_{3}\right)$ (that is the coefficient of degree 12) is given by

$$
\begin{aligned}
& 16(p+1)^{5}\left(p^{2}+4 p-1\right)\left((p+2)(m-2 p-2)+p^{2}+p\right)^{2} \times \\
& \left(8(m-2 p-2)^{2}+16 p(m-2 p-2)+p^{3}+5 p^{2}+3 p-1\right)>0 .
\end{aligned}
$$

We claim that there exists $x_{3}{ }^{0}>0$ such that $h_{1}\left(x_{3}{ }^{0}\right)<0$. Then we see that there exist at least two positive solutions of the equation $h_{1}\left(x_{3}\right)=0$. For fixed $m$ we divide $p$ into the following 4 cases:

(1) the case when $2 \leq p \leq \frac{m}{4} \quad$ (2) the case when $\frac{m}{4}+1 \leq p \leq \frac{m}{3}$

(3) the case when $\frac{m}{3}+1 \leq p \leq \frac{3}{8} m \quad$ (4) the case when $\frac{3}{8} m+1 \leq p \leq \frac{m-3}{2}$.

Case (1). We put $x_{3}{ }^{0}=\frac{1}{2}+\frac{13}{16 m}-\frac{5 p}{16 m}$. We claim that for $3 \leq p \leq \frac{m}{4}, h_{1}\left(x_{3}{ }^{0}\right)<0$. Consider the value $h_{1}\left(\frac{1}{2}+\frac{13}{16 m}-\frac{5 p}{16 m}\right)$. We see that

$$
h_{1}\left(\frac{1}{2}+\frac{13}{16 m}-\frac{5 p}{16 m}\right)=-\frac{1}{17592186044416 m^{12}} G_{1}(m, p),
$$


where $G_{1}(m, p)$ is a polynomial of $m$ and $p$ with integer coefficients of degree 23 for $m$. This is given by

$$
G_{1}(m, p)=\sum_{k=0}^{23} a_{k}(p)(m-4 p)^{k},
$$

where $a_{k}(p)$ are polynomials of $p$ with integer coefficients. We expand each $a_{k}(p)$ by $p-3$ and we see that these are polynomials of $p-3$ with positive integer coefficients. For example, we have

$$
\begin{aligned}
& a_{0}(p)=178237127754237399126183(p-3)^{26}+13265901008221449505213854(p-3)^{25} \\
& +468508725568700912318217147(p-3)^{24}+10476580337328823577318977524(p-3)^{23}+\cdots \\
& +5459366557078936923770981445634359296(p-3)^{2}+102099050788760068306158510149730304(p-3) \\
& +9153796573419518258107893315272704 .
\end{aligned}
$$

Thus we see that for $p \geq 3$ and $m-4 p \geq 0, G_{1}(m, p)$ is positive. For $p=2$, we have that

$$
\begin{aligned}
& G_{1}(m, 2)=4947802324992 m^{23}-238731462180864 m^{22}+4683833634979840 m^{21} \\
& -46281577591734272 m^{20}+202492806617366528 m^{19}+347599071281676288 m^{18} \\
& -9310980063572787200 m^{17}+52574830445585235968 m^{16}-150817861595192885248 m^{15} \\
& +203948015024640884736 m^{14}+24172844877444808704 m^{13}-443862342994666192896 m^{12} \\
& +385551424965459050496 m^{11}+234121922151674609664 m^{10}-374049639831778762752 m^{9} \\
& -75293632155127080960 m^{8}+131014157184763195392 m^{7}+41471745352938388224 m^{6} \\
& -4230801125626406400 m^{5}-3773984791973043456 m^{4}-724276391563682496 m^{3} \\
& -67160272036488624 m^{2}-3137789825780976 m-59372964780228 .
\end{aligned}
$$

By expanding $G_{1}(m, 2)$ by $m-13$, we obtain that

$$
\begin{aligned}
& G_{1}(m, 2)=4947802324992(m-13)^{23}+1240661432991744(m-13)^{22}+147959819460935680(m-13)^{21} \\
& +11163907197560160256(m-13)^{20}+598016367241983950848(m-13)^{19}+\cdots \\
& +863786663385687333093846137528883728(m-13)^{2}+505363778599954771716113864626795760(m-13) \\
& +91549876964199619601498344378250268 .
\end{aligned}
$$

Thus we obtain that $G_{1}(m, 2)>0$ for $m \geq 13$.

Case (2). We put $x_{3}{ }^{0}=\frac{19}{50}$. We claim that for $\frac{m}{4}+2 \leq p \leq \frac{m}{3}, h_{1}\left(x_{3}{ }^{0}\right)<0$. Consider the value $h_{1}\left(\frac{19}{50}\right)$ for $p=\frac{m}{4}+s$ where $s$ is a positive integer. We see that

$$
h_{1}\left(\frac{19}{50}\right)=-\frac{1}{4096000000000000000000000000} G_{2}(m, s),
$$

where $G_{2}(m, s)$ is a polynomial of $m$ and $s$ with integer coefficients of degree 14 for $m$. We see that the polynomial $G_{2}(m, s)$ is of the form given by

$$
G_{2}(m, s)=\sum_{k=0}^{14} b_{k}(s)(m-12 s)^{k}
$$

where $b_{k}(s)$ are polynomials of $p$ with integer coefficients. We see that each $b_{k}(s)$ is a polynomial of $s-2$ with positive integer coefficients. For example, we have

$$
\begin{aligned}
& b_{0}(s)=1506786986744786694940025493563375616(s-2)^{14}+36998433298516093734987416088141103104(s-2)^{13} \\
& +416136307149363560959687947416881856512(s-2)^{12}+2842633983062558684587917475200569966592(s-2)^{11}+\cdots \\
& +57949158057391373824741968217195103125504(s-2)^{2}+14661733981405213296399078855588634951680(s-2) \\
& +1759509038746291790869701479717803130880 .
\end{aligned}
$$

Thus we see that, for $s \geq 2$ and $m-12 s \geq 0, G_{2}(m, s)$ is positive. Note that $2 \leq s \leq m / 12$ and thus $p \leq m / 4+m / 12=m / 3$.

For $s=1$, that is $p=m / 4+1$, we consider $h_{1}\left(\frac{21}{50}\right)$. Then we see that

$$
h_{1}\left(\frac{21}{50}\right)=-\frac{9}{4096000000000000000000000000} H_{2}(m, 1)
$$


where

$$
\begin{aligned}
& H_{2}(m, 1)=1054050555264795935559 m^{14}-25389815873416469983512 m^{13}-966477257093633919382992 m^{12} \\
& +46723891545491804668385536 m^{11}-692407952396029127541554176 m^{10}+2877161421721862355752550400 m^{9} \\
& +38703106006797200198212583424 m^{8}-637677740991893898125100711936 m^{7} \\
& +4388384700195221430188604653568 m^{6}-17925423535989571036538101301248 m^{5} \\
& +47001713463749690546636544016384 m^{4}-80077090514342627715801111592960 m^{3} \\
& +85882621352257394136232639856640 m^{2}-52734119195798771677768817049600 m \\
& +14142949365346227611634342297600 .
\end{aligned}
$$

We see that

$$
\begin{aligned}
& H_{2}(m, 1)=1054050555264795935559(m-13)^{14}+166447385184776390288226(m-13)^{13} \\
& +10952887349716279346365341(m-13)^{12}+404197548045208433956000372(m-13)^{11}+\cdots \\
& +15512402577878159456329789083376125(m-13)^{2}+19060854444302720753441098137077730(m-13) \\
& +1049301029441675428621431247614375
\end{aligned}
$$

Thus $H_{2}(m, 1)$ is positive for $m \geq 13$.

Case (3). We put $x_{3}{ }^{0}=\frac{1}{3}$. We claim that for $\frac{m}{3}+1 \leq p \leq \frac{3}{8} m, h_{1}\left(x_{3}{ }^{0}\right)<0$. Consider the value $h_{1}\left(\frac{1}{3}\right)$ for $p=\frac{m}{3}+s$ where $s$ is a positive integer. We see that

$$
h_{1}\left(\frac{1}{3}\right)=-\frac{1}{2541865828329} G_{3}(m, s),
$$

where $G_{3}(m, s)$ is a polynomial of $m$ and $s$ with integer coefficients of degree 14 for $m$. We see that the polynomial $G_{3}(m, s)$ is of the form given by

$$
G_{3}(m, s)=\sum_{k=0}^{14} c_{k}(s)(m-24 s)^{k},
$$

where $c_{k}(s)$ are polynomials of $p$ with integer coefficients. We see that each $c_{k}(s)$ is a polynomial of $s-1$ with positive integer coefficients. For example, we have

$$
\begin{aligned}
& c_{0}(s)=2688886554248702829059568(s-1)^{14}+28773011904669834888459456(s-1)^{13} \\
& +141508288769505404340266208(s-1)^{12}+425988148380665862862038816(s-1)^{11}+\cdots \\
& +19077956371801372323151872(s-1)^{2}+2309278434711832223023104(s-1) \\
& +108869460718905531039744
\end{aligned}
$$

Thus we see that for $s \geq 1$ and $m-24 s \geq 0, G_{3}(m, s)$ is positive. Note that $1 \leq s \leq m / 24$ and thus $m / 3+1 \leq p \leq m / 3+m / 24=3 m / 8$.

Case (4). We put $q=m / 2-p$ and $x_{3}{ }^{0}=\frac{4 q}{m}-\frac{4}{m}-8\left(\frac{q}{m}\right)^{2}+16 \frac{q}{m^{2}}$. We claim that, for $2 \leq q \leq \frac{1}{8} m$, that is, $\frac{3}{8} m \leq p \leq \frac{m}{2}-2, h_{1}\left(x_{3}{ }^{0}\right)<0$. Consider the value $h_{1}\left(\frac{4 q}{m}-\frac{4}{m}-8\left(\frac{q}{m}\right)^{2}+16 \frac{q}{m^{2}}\right)$. We see that

$$
h_{1}\left(\frac{4 q}{m}-\frac{4}{m}-8\left(\frac{q}{m}\right)^{2}+16 \frac{q}{m^{2}}\right)=-\frac{16}{m^{24}} G_{4}(m, q),
$$

where $G_{4}(m, q)$ is a polynomial of $m$ and $q$ with integer coefficients of degree 30 for $m$. We see that the polynomial $G_{4}(m, q)$ is of the form given by

$$
G_{4}(m, q)=\sum_{k=0}^{30} u_{k}(q)(m-8 q)^{k},
$$

where $u_{k}(q)$ are polynomial of $q$ with integer coefficients. We see that each $u_{k}(q)$ is a polynomial of $q-2$ with positive integer coefficients. For example, we have

$$
\begin{aligned}
& u_{0}(q)=26951178076734183104839680(q-2)^{38}+2212338096952683249388224512(q-2)^{37} \\
& +83992678988503465460710244352(q-2)^{36}+1995946208003865782253049085952(q-2)^{35}+\cdots \\
& +229210217524459650051376283663636365312(q-2)^{2}+22366794926378575054826400937697869824(q-2) \\
& +1012881211339770900930920976868179968
\end{aligned}
$$


Thus we obtain that $G_{4}(m, q)>0$ for $2 \leq q \leq m / 8$, that is, for $\frac{3}{8} m \leq p \leq \frac{m}{2}-2$.

Note that, for the case when $m=2 \ell$, we have $p \leq \ell-3=m / 2-3<\frac{m-3}{2}$, and for the case when $m=2 \ell+1$, we have $p \leq \ell-1=(m-1) / 2-1$. We consider the case $p=\ell-1$ where $m=2 \ell+1$. We put $x_{3}{ }^{0}=\frac{1}{\ell}-\frac{1}{4 \ell^{2}}$. We see that

$$
h_{1}\left(\frac{1}{\ell}-\frac{1}{4 \ell^{2}}\right)=-\frac{1}{1048576 \ell^{19}} H_{4}(\ell)
$$

where

$$
\begin{aligned}
& H_{4}(\ell)=28311552 \ell^{25}+127926272 \ell^{24}-3676700672 \ell^{23}+25529024512 \ell^{22}-99774468096 \ell^{21} \\
& +251607146496 \ell^{20}-413969008640 \ell^{19}+386872995840 \ell^{18}+11909161728 \ell^{17}-698697895936 \ell^{16} \\
& +1310915822464 \ell^{15}-1514173731328 \ell^{14}+1280110627808 \ell^{13}-839682485472 \ell^{12}+439251246304 \ell^{11} \\
& -185919232072 \ell^{10}+64111849503 \ell^{9}-18030414660 \ell^{8}+4116853866 \ell^{7}-755179592 \ell^{6} \\
& +109349551 \ell^{5}-12168116 \ell^{4}+999284 \ell^{3}-56776 \ell^{2}+1984 \ell-32 .
\end{aligned}
$$

We see that

$$
\begin{aligned}
& H_{4}(\ell)=28311552(\ell-3)^{25}+2251292672(\ell-3)^{24}+81975181312(\ell-3)^{23}+1847752916992(\ell-3)^{22}+\cdots \\
& +287547059522662005140(\ell-3)^{3}+97552658701667320160(\ell-3)^{2}+21045285340535234500(\ell-3) \\
& +2167673762760385300
\end{aligned}
$$

so $h_{1}\left(\frac{1}{\ell}-\frac{1}{4 \ell^{2}}\right)<0$ for $\ell \geq 3$.

We now take a lexicographic order $>$ with $y>x_{3}>x_{4}>x_{2}$ for a monomial ordering on $R$. Then we see that a Gröbner basis for the ideal $I_{1}$ contains the following polynomial $h_{2}\left(x_{2}\right)$ of degree 12 :

$$
\begin{aligned}
& h_{2}\left(x_{2}\right)=16(p+1)^{5}\left(p^{2}+4 p-1\right)\left(p^{3}+5 p^{2}-16 m p+35 p+8 m^{2}-32 m+31\right) x_{2}{ }^{12} \\
& -32(m-2)(p+1)^{4}\left(p^{5}+m p^{4}+9 p^{4}-26 m p^{3}+88 p^{3}+16 m^{2} p^{2}-172 m p^{2}+308 p^{2}+56 m^{2} p-222 m p\right. \\
& \left.+207 p-8 m^{2}+35 m-37\right) x_{2}{ }^{11}+\cdots \\
& -4(m-2)^{4}\left(m^{6}-2 p m^{5}-10 m^{5}-3 p^{2} m^{4}+8 p m^{4}+31 m^{4}+8 p^{3} m^{3}+40 p^{2} m^{3}+50 p m^{3}-18 m^{3}-2 p^{4} m^{2}\right. \\
& -56 p^{3} m^{2}-176 p^{2} m^{2}-288 p m^{2}-54 m^{2}-3 p^{5} m+23 p^{4} m+86 p^{3} m+226 p^{2} m+397 p m+39 m \\
& \left.+p^{6}-6 p^{5}-23 p^{4}+52 p^{3}+27 p^{2}-94 p+43\right) x_{2} \\
& +(m-2)^{4}(m-p-3)\left(m^{2}-p m-7 m-p^{2}+6 p+11\right)\left(m^{3}-2 p m^{2}-2 m^{2}+p^{3}-3 p^{2}+3 p-1\right) .
\end{aligned}
$$

We claim that the equation $h_{2}\left(x_{2}\right)=0$ has at least one positive real root. We write

$$
h_{2}\left(x_{2}\right)=\sum_{k=0}^{12} b_{k}(m, p)(-1)^{k} x_{2}{ }^{k} .
$$

Then $b_{k}(m, p)$ are polynomial of $m$ and $p$ with integer coefficients. It is enough to see that $b_{k}(m, p)$ are positive. Note that, if we denote by $n_{k}$ the degree of $b_{k}(m, p)$ with respect to $m$, then we see that $n_{12}=2$, $n_{11}=3, n_{10}=4, n_{9}=5, n_{8}=6, n_{7}=6, n_{6}=6, n_{5}=6, n_{4}=6, n_{3}=6, n_{2}=6, n_{1}=6, n_{0}=6$. We see that each polynomial $b_{k}(m, p)$ is of the form given by

$$
b_{k}(m, p)=\sum_{j=0}^{n_{k}} u_{j}^{k}(p)(m-2 p-3)^{j}
$$

where $u_{j}^{k}(p)$ are polynomials of $p$ with integer coefficients. Now we see that each $u_{j}^{k}(p)$ is a polynomial of $p-2$ with positive integer coefficients. For example, we have

$$
\begin{aligned}
& u_{0}^{0}(p)=16(p-2)^{10}+384(p-2)^{9}+4360(p-2)^{8}+30712(p-2)^{7}+146905(p-2)^{6}+491510(p-2)^{5} \\
& +1149975(p-2)^{4}+1839750(p-2)^{3}+1913125(p-2)^{2}+1162500(p-2)+312500 .
\end{aligned}
$$

Thus we obtain that $b_{k}(m, p)>0$ for $2 \leq p \leq(m-3) / 2$. From (46), we see that there exists a real solution for the equation $h_{2}\left(x_{2}\right)=0$ and hence, it is a positive solution of $h_{2}\left(x_{2}\right)=0$. 
We take a lexicographic order $>$ with $y>x_{3}>x_{2}>x_{4}$ for a monomial ordering on $R$. Then we see that a Gröbner basis for the ideal $I_{1}$ contains the following polynomial $h_{3}\left(x_{4}\right)$ of degree 12 :

$$
\begin{aligned}
& h_{3}\left(x_{4}\right)=(m-p-1)^{4}(p-1)^{4}\left(-p^{2}+m p-5 p+2 m-4\right)^{2}\left(4 p^{4}-4 m p^{3}+16 p^{3}+m^{2} p^{2}-8 m p^{2}\right. \\
& \left.+16 p^{2}-4 m^{2} p+16 m p-16 p+2 m^{3}-12 m^{2}+24 m-16\right) x_{4}{ }^{2} \\
& -2(m-2)(m-p-1)^{3}(p-1)^{3}\left(-p^{2}+m p-5 p+2 m-4\right)\left(16 p^{7}-52 m p^{6}+192 p^{6}+60 m^{2} p^{5}-488 m p^{5}\right. \\
& +872 p^{5}-29 m^{3} p^{4}+412 m^{2} p^{4}-1624 m p^{4}+1872 p^{4}+5 m^{4} p^{3}-118 m^{3} p^{3}+816 m^{2} p^{3}-2056 m p^{3} \\
& +1688 p^{3}-7 m^{4} p^{2}+37 m^{3} p^{2}-100 m^{2} p^{2}+212 m p^{2}-208 p^{2}+6 m^{5} p-90 m^{4} p+542 m^{3} p-1588 m^{2} p \\
& \left.+2248 m p-1232 p+8 m^{5}-96 m^{4}+448 m^{3}-1024 m^{2}+1152 m-512\right) x_{4}{ }^{11}+\cdots \\
& -256(m-2)^{4}(m-2 p)^{2}(p-1)^{3}\left(m^{7}-2 p m^{6}-14 m^{6}-4 p^{2} m^{5}+24 p m^{5}+76 m^{5}+14 p^{3} m^{4}+34 p^{2} m^{4}-110 p m^{4}\right. \\
& -194 m^{4}-14 p^{4} m^{3}-130 p^{3} m^{3}-6 p^{2} m^{3}+202 p m^{3}+204 m^{3}+4 p^{5} m^{2}+168 p^{4} m^{2}+240 p^{3} m^{2}-456 p^{2} m^{2}+12 p m^{2} \\
& +32 m^{2}+5 p^{6} m-90 p^{5} m-425 p^{4} m+180 p^{3} m+1043 p^{2} m-506 p m-207 m-4 p^{7}+6 p^{6}+160 p^{5}+306 p^{4} \\
& \left.-436 p^{3}-598 p^{2}+472 p+94\right) x+256(m-2)^{4}(m-2 p)^{2}(m-p-3)(p-1)^{4}\left(m^{2}-p m-7 m-p^{2}+6 p+11\right) \times \\
& \left(m^{3}-2 p m^{2}-2 m^{2}+p^{3}-3 p^{2}+3 p-1\right) .
\end{aligned}
$$

We claim that the equation $h_{3}\left(x_{4}\right)=0$ has at least one positive real root. We write

$$
h_{3}\left(x_{4}\right)=\sum_{k=0}^{12} c_{k}(m, p)(-1)^{k} x_{4}{ }^{k} .
$$

Then $c_{k}(m, p)$ are polynomial of $m$ and $p$ with integer coefficients. It is enough to see that $c_{k}(m, p)$ are positive. Note that, if we denote by $n_{k}$ the degree of $c_{k}(m, p)$ with respect to $m$, then we see that $n_{12}=9$, $n_{11}=10, n_{10}=12, n_{9}=12, n_{8}=13, n_{7}=13, n_{6}=13, n_{5}=13, n_{4}=13, n_{3}=13, n_{2}=13, n_{1}=13$, $n_{0}=12$. We see that each polynomial $c_{k}(m, p)$ is of the form given by

$$
c_{k}(m, p)=\sum_{j=0}^{n_{k}} v_{j}^{k}(p)(m-2 p-3)^{j},
$$

where $v_{j}^{k}(p)$ are polynomials of $p$ with integer coefficients. Now we see that each $v_{j}^{k}(p)$ is a polynomial of $p-2$ with positive integer coefficients. For example, we have

$$
\begin{aligned}
& v_{0}^{0}(p)=36864(p-2)^{14}+1032192(p-2)^{13}+13805568(p-2)^{12}+116398080(p-2)^{11} \\
& +685359360(p-2)^{10}+2951944704(p-2)^{9}+9503200512(p-2)^{8}+23056224768(p-2)^{7} \\
& +42128455680(p-2)^{6}+57473072640(p-2)^{5}+57485318400(p-2)^{4}+40820544000(p-2)^{3} \\
& +19441440000(p-2)^{2}+5558400000(p-2)+720000000 .
\end{aligned}
$$

Thus we obtain that $c_{k}(m, p)>0$ for $2 \leq p \leq(m-3) / 2$. From (46), we see that there exists a real solution for the equation $h_{3}\left(x_{4}\right)=0$ and hence, it is a positive solution of $h_{3}\left(x_{4}\right)=0$.

Therefore we obtain the following

Theorem 7.3. The system of equations (45) has at least two positive solutions. Thus the flag manifold $M=\mathrm{SO}(m) /(\mathrm{U}(1) \times \mathrm{U}(p) \times \mathrm{SO}(m-2(p+1))$ admits at least two $\mathrm{SO}(m)$-invariant non-Kähler Einstein metrics for any $p \geq 3$, and for $p=2$ when $m \geq 13$.

For $p=2$ it is possible to use a similar analysis as before and sharpen the above result. In fact, as for the case (1) we see that

$$
h_{1}\left(\frac{1}{2}\left(\frac{20 m}{33}-\frac{493}{198}\right)\right)=-\frac{1}{173857445425479232434733056} K_{1}(m),
$$

where $K_{1}(m)$ is a polynomial of $m$ with integer coefficients of degree 16 . By expanding $K_{1}(m)$ by $m-11$, we obtain that

$$
\begin{aligned}
& K_{1}(m)=3583035271261716480000(m-11)^{16}+511250673311500625510400(m-11)^{15} \\
& +31818296826635532065832960(m-11)^{14}+1173386825534099922378817536(m-11)^{13} \\
& +29027304327079432810844872704(m-11)^{12}+513765537329981469527636631552(m-11)^{11}+\cdots \\
& +99971319095011447419581739885675068(m-11)+15879235006092866105410345517930679 .
\end{aligned}
$$

Thus we obtain that $K_{1}(m)>0$ for $m \geq 11$.

We also see that 


$$
\begin{aligned}
& h_{1}(1)=(m-5)^{2}\left(m^{2}-9 m+19\right)^{2}\left((m-10)^{7}+24(m-10)^{6}+211(m-10)^{5}+850(m-10)^{4}\right. \\
& \left.+1620(m-10)^{3}+1448(m-10)^{2}+544(m-10)+72\right) .
\end{aligned}
$$

Thus we obtain that $h_{1}(1)>0$ for $m \geq 10$.

Together with the above result, we obtain the following:

Theorem 7.4. The flag manifolds $\mathrm{SO}(2 \ell+1) /(\mathrm{U}(1) \times \mathrm{U}(2) \times \mathrm{SO}(2 \ell-5))(\ell \geq 6)$ and $\mathrm{SO}(2 \ell) /(\mathrm{U}(1) \times$ $\mathrm{U}(2) \times \mathrm{SO}(2(\ell-3)))(\ell \geq 7)$ admit at least four invariant non-Kähler Einstein metrics.

Moreover, for small values of $\ell$ and $p$ it is possible to obtain the precise number of invariant Einstein metrics depending on type $B_{\ell}$ and $D_{\ell}$ as follows.

Table 4. The number of non-isometric homogeneous Einstein metrics

\begin{tabular}{|c|c|c|c|l|}
\hline$(\ell, p)$ & $\begin{array}{c}\text { Non-Kähler Einstein } \\
\text { of type a }\end{array}$ & $\begin{array}{c}\text { Non-Kähler Einstein } \\
\text { of type b }\end{array}$ & Kähler Einstein & Generalized flag manifold \\
\hline$(3,2)$ & 2 & 1 & 2 & $\mathrm{SO}(7) / \mathrm{U}(1) \times \mathrm{U}(2)$ \\
\hline$(4,2)$ & 4 & 2 & 2 & $\mathrm{SO}(9) / \mathrm{U}(1) \times \mathrm{U}(2) \times \mathrm{SO}(3)$ \\
\hline$(4,3)$ & 2 & 1 & 2 & $\mathrm{SO}(9) / \mathrm{U}(1) \times \mathrm{U}(3)$ \\
\hline$(5,2)$ & 4 & 2 & 2 & $\mathrm{SO}(11) / \mathrm{U}(1) \times \mathrm{U}(2) \times \mathrm{SO}(5)$ \\
\hline$(5,3)$ & 2 & 1 & 2 & $\mathrm{SO}(11) / \mathrm{U}(1) \times \mathrm{U}(4)$ \\
\hline$(5,4)$ & 2 & 1 & 2 & $\mathrm{SO}(13) / \mathrm{U}(1) \times \mathrm{U}(2) \times \mathrm{SO}(7)$ \\
\hline$(6,2)$ & 4 & 2 & 2 & $\mathrm{SO}(13) / \mathrm{U}(1) \times \mathrm{U}(3) \times \mathrm{SO}(5)$ \\
\hline$(6,3)$ & 2 & 2 & 2 & $\mathrm{SO}(13) / \mathrm{U}(1) \times \mathrm{U}(4) \times \mathrm{SO}(3)$ \\
\hline$(6,4)$ & 2 & 1 & 2 & $\mathrm{SO}(13) / \mathrm{U}(1) \times \mathrm{U}(5)$ \\
\hline$(6,5)$ & 2 & 1 & 2 & $\mathrm{SO}(12) / \mathrm{U}(1) \times \mathrm{U}(2) \times \mathrm{SO}(6)$ \\
\hline$(5,2)$ & 4 & 2 & 2 & $\mathrm{SO}(12) / \mathrm{U}(1) \times \mathrm{U}(3) \times \mathrm{SO}(4)$ \\
\hline$(6,2)$ & 4 & 2 & 2 & $\mathrm{SO}(14) / \mathrm{U}(1) \times \mathrm{U}(2) \times \mathrm{SO}(8)$ \\
\hline$(6,3)$ & 2 & 2 & 2 & $\mathrm{SO}(14) / \mathrm{U}(1) \times \mathrm{U}(3) \times \mathrm{SO}(6)$ \\
\hline$(7,2)$ & 4 & 2 & $\mathrm{SO}(14) / \mathrm{U}(1) \times \mathrm{U}(4) \times \mathrm{SO}(4)$ \\
\hline$(7,3)$ & 4 & 2 & 2 &
\end{tabular}

Non-Kähler Einstein metric of type a means that the metric of the form with $x_{1}=x_{5}$ and Non-Kähler Einstein metric of type $\mathrm{b}$ means that the metric of the form with $x_{1} \neq x_{5}$.

We conjecture that for the classical flag manifolds studied in the present work the total number of non isometric invariant Einstein metrics is precisely five, six or eight. Note that two of them are Kähler-Einstein metrics.

\section{REFERENCES}

[Ale1] D. V. Alekseevsky: Flag manifolds, in Sbornik Radova, 11th Jugoslav. Geom. Seminar. Beograd 6 (14) (1997) 3-35.

[Ale2] D. V. Alekseevsky: Isotropy representations of flag manifolds, in: Procceding 17 Winter Srni School, editors, J. Slovak et al., Suppl. Rendic. di Circ. Matem. di Palermo (1998) 12-24.

[AlAr] D. V. Alekseevsky and A. Arvanitoyeorgos: Riemannian flag manifolds with homogeneous geodesics, Trans. Amer. Math. Soc. 359 (8) (2007) 3769-3789.

[AlPe] D. V. Alekseevsky and A. M. Perelomov: Invariant Kähler-Einstein metrics on compact homogeneous spaces, Funct. Anal. Appl. 20 (3) (1986) 171-182.

[AnCh] S. Anastassiou, I. Chrysikos, The Ricci flow approach to homogeneous Einstein metrics on flag manifolds, J. Geom. Phys. 61 (2011), 1587-1600.

[Arv] A. Arvanitoyeorgos: New invariant Einstein metrics on generalized flag manifolds, Trans. Amer. Math. Soc. 337 (2) (1993) 981-995.

[ACh1] A. Arvanitoyeorgos and I. Chrysikos: Motion of charged particles and homogeneous geodesics in Kähler C-spaces with two isotropy summands, Tokyo J. Math. 32 (2) (2009), 487-500.

[ACh2] A. Arvanitoyeorgos and I. Chrysikos: Invariant Einstein metrics on generalized flag manifolds with two isotropy summands, J. Aust. Math. Soc. 90 (2) (2011) 237-251. 
[ACh3] A. Arvanitoyeorgos and I. Chrysikos: Invariant Einstein metrics on generalized flag manifolds with four isotropy summands, Ann. Glob. Anal. Geom. 37 (2) (2010) 185-219.

[ACS1] A. Arvanitoyeorgos, I. Chrysikos and Y. Sakane: Complete description of invariant Einstein metrics on the generalized flag manifold $S O(2 n) / U(p) \times U(n-p)$, Ann Glob Anal Geom. 38 (4) (2010) 413-438.

[ACS2] A. Arvanitoyeorgos, I. Chrysikos and Y. Sakane: Honogeneous Einstein metrics on the generalized flag manifold $S p(n) /(U(p) \times U(n-p))$, Differential Geom. Appl. 29 (2011) S16-S27.

[ACS3] A. Arvanitoyeorgos, I. Chrysikos and Y. Sakane: Homogeneous Einstein metrics on generalized flag manifolds $S p(n) /(U(p) \times U(q) \times S p(n-p-q))$, Proceedings of the 2nd International Colloquium on Differential Geometry and its Related Fields, World Scientific Publishing Co. Pte. Ltd, Veliko Tarnovo, September 6-10 2010, 1-24.

[ACS4] A. Arvanitoyeorgos, I. Chrysikos and Y. Sakane: Homogeneous Einstein metrics on $G_{2} / T$, Proc. Amer. Math. Soc. (in press).

[ACS5] A. Arvanitoyeorgos, I. Chrysikos and Y. Sakane: The isometry problem for honogeneous Einstein metrics on $S p(n) /(U(p) \times U(n-p))$, submitted.

[AMS] A. Arvanitoyeorgos, K. Mori and Y. Sakane: Einstein metrics on compact Lie groups which are not naturally reductive, Geom. Dedicata (in press).

[Be] A. L. Besse: Einstein Manifolds, Springer-Verlag, Berlin, 1986.

[BFR] M. Bordeman, M. Forger and H. Römer: Homogeneous Kähler manifolds: paving the way towards new supersymmetric sigma models, Comm. Math. Phys. 102 (1986) 604-647.

[Bor] A. Borel and F. Hirzebruch: Characteristics classes and homogeneous spaces I, Amer. J. Math. 80 (1958) $458-538$.

[Bou] N. Bourbaki-Éléments De Mathématique: Groupes Et Algèbres De Lie, Chapitres 4, 5 et 6 Masson Publishing, Paris, 1981.

[Chr1] I. Chrysikos: Homogeneous Einstein Metrics on Generalized Flag Manifolds, Ph.D Thesis, University of Patras, 2011 (electronic version : http://nemertes.lis.upatras.gr/jspui/handle/10889/4418?locale=en)

[Chr2] I. Chrysikos: Flag manifold, symmetric t-triples and Einstein metrics, Differential Geom. Appl. (accepted) (arXiv: 1010.3992v2.)

[ChSa] I. Chrysikos and Y. Sakane: On the classification of homogeneous Einstein metrics on generalized flag manifolds with $b_{2}(M)=1$ (submitted).

[DSN] E. C. F. Dos Santos and C. J. C. Negreiros: Einstein metrics on flag manifolds, Revista Della, Unión Mathemática Argetina, 47 (2) (2006) 77-84.

[FrdV] H. Freudenthal and H. de Vries: Linear Lie Groups, Academic Press, New York, 1969.

[GOV] V.V. Gorbatzevich - A.L. Onishchik - E.B. Vinberg: Structure of Lie Groups and Lie Algebras, Encycl. of Math. Sci. v41, Lie Groups and Lie Algebras-3, Springer-Verlag.

[Hel] S. Helgason: Differential Geometry, Lie Groups and Symmetric Spaces, Academic Press, New York 1978

[Kim] M. Kimura: Homogeneous Einstein metrics on certain Kähler C-spaces, Adv. Stud. Pure Math. 18-I (1990) 303-320.

[Nis] M. Nishiyama: Classification of invariant complex structures on irreducible compact simply connected coset spaces, Osaka J. Math. 21 (1984) 39-58.

[ON] B. O'Neill: The fundamental equation of a submersion, Michigan Math. J. 13 (1966) 459-469.

[Sak1] Y. Sakane: Homogeneous Einstein metrics on principal circle bundles II, in Differential Geometry, Proceedings of the symposioum in honour of Professor Su Buchin on his 90th birthday (Editors: C. H. Gu, H. S. Hu, Y. L. Xin), World Scientific Publishing, (1993) 177-186.

[Sak2] Y. Sakane: Homogeneous Einstein metrics on flag manifolds, Lobachevskii J. Math. (4) (1999) 71-87.

[PaSa] J-S. Park and Y. Sakane: Invariant Einstein metrics on certain homogeneous spaces, Tokyo J. Math. 20 (1) (1997) 51-61.

[WaZi] M. Wang and W. Ziller: Existence and non-excistence of homogeneous Einstein metrics, Invent. Math. 84 (1986) $177-194$.

University of Patras, Department of Mathematics, GR-26500 Rion, Greece

E-mail address: arvanito@math.upatras.gr

Masaryk University, Department of Mathematics and Statistics, Masaryk University, Brno 611 37, Czech RePUBLIC

E-mail address: chrysikosi@math.muni.cz

Osaka University, Department of Pure and Applied Mathematics, Graduate School of Information Science and Technology, Toyonaka, Osaka 560-0043, Japan

E-mail address: sakane@math.sci.osaka-u.ac.jp 